\title{
The Nilpotent Subvariety of the Vector Space Associated to a Symmetric Pair
}

By

\author{
Jiro SEKIGUCHI*
}

\section{Introduction}

Let $\mathfrak{g}$ be a semisimple Lie algebra over $\boldsymbol{C}$ and let $\theta$ be a complex linear involution of $\mathfrak{g}$. Then we obtain a direct sum decomposition $\mathfrak{g}=\mathfrak{f}+V$, where $\mathfrak{f}=\{X \in \mathfrak{g} ; \theta(X)=X\} \quad$ and $V=\{X \in \mathfrak{g} ; \theta(X)=-X\}$. In this paper the pair $(\mathfrak{g}, \mathfrak{f})$ is called a symmetric pair and $V$ is the vector space associated to it. Our main concern is the nilpotent subvariety $\mathscr{N}(V)$ of $V$. An element $X$ of $V$ is contained in $\mathscr{N}(V)$ if and only if $a d_{\mathfrak{B}}(X)$ is nilpotent.

We first describe the motivation of the study.

Let us assume that $g$ is a direct sum of another semisimple Lie algebra $\mathfrak{g}^{\prime}$, that is, $\mathfrak{g}=\mathfrak{g}^{\prime} \oplus \mathfrak{g}^{\prime}$ and the involution $\theta$ is defined by $\theta(X, Y)=(Y, X)\left(X, Y \in \mathfrak{g}^{\prime}\right)$. Then we obtain a symmetric pair $(\mathfrak{g}, \mathfrak{f})$ and the vector space $V$ for $\theta$. In this case, $\mathfrak{f}$ and $V$ are obviously isomorphic to $\mathfrak{g}^{\prime}$ itself. Furthermore, the nilpotent subvariety $\mathscr{N}(V)$ of $V$ is identified with that of $\mathrm{g}^{\prime}$. Then due to the celebrated result of Brieskorn [Br], we find that if $\mathfrak{g}^{\prime}$ is simple of type $A_{l}, D_{l}$ or $E_{l}$, the generic singularity of $\mathscr{N}(V)$ is smoothly equivalent to the rational double point of the corresponding type. In the case when $g^{\prime}$ is simple of type $B_{l}, C_{l}, F_{4}$ or $G_{2}$, a similar result is obtaind by Slodowy [SI].

The results of Brieskorn and Slodowy naturally lead us to the problem of the determination of the generic singularities of $\mathscr{N}(V)$ for a general symmetric pair $(\mathfrak{g}, \mathfrak{f})$. When $(\mathfrak{g}, \mathfrak{f})$ is of the normal type in the sense of [S-S], we obtain a result similar to that of Brieskorn. But in general, it seems to be hard to determine the generic singularities. To explain the reasons, we mention some

Communicated by M. Sato, February 1, 1983.

* Faculty of Science, Tokyo Metropolitan University, Setagaya-ku, Tokyo 158, Japan. 
differences between the nilpotent subvariety $\mathscr{N}(V)$ and that of a simple Lie algebra.

Let $\mathscr{N}_{\mathrm{B}}$ be the nilpotent subvariety of a simple Lie algebra $\mathrm{g}$. If $\left(\mathscr{N}_{\mathrm{B}}\right)^{\prime}$ denotes the totality of the smooth points of $\mathscr{N}_{\mathrm{B}}$, then $\left(\mathscr{N}_{\mathrm{B}}\right)^{\prime}$ is a single $G$-orbit. Here $G$ is the adjoint group of $g$. Furthermore, if $\left(\mathscr{N}_{\mathrm{B}}\right)_{\operatorname{sing}}^{\prime}$ denotes the totality of the smooth points of $\mathscr{N}_{\mathrm{B}}-\left(\mathscr{N}_{\mathrm{B}}\right)^{\prime}$, then $\left(\mathscr{N}_{\mathrm{B}}\right)_{\text {sing }}^{\prime}$ is also a single $G$-orbit. Usually an element of $\left(\mathscr{N}_{\mathrm{B}}\right)^{\prime}$ (resp. $\left(\mathscr{N}_{\mathrm{B}}\right)_{\text {sing }}^{\prime}$ ) is called a regular (resp. subregular) nilpotent element of g. Kostant [Ko, 1, 2] studied the regular nilpotent elements deeply and Steinberg [St] gave several characterizations of subregular nilpotent elements among general nilpotent elements. Needless to say, Brieskorn $[\mathrm{Br}]$ pointed out the importance of subregular nilpotent elements in the study of the singularities as stated above. Moreover as for the orbital structure of the nilpotent variety $\mathscr{N}_{8}$, ever since Dynkin's paper [D], several authors (cf. [Sp-St], [B-C], etc.) have dealt with the topics and therefore at the present the structure is made clear so that the results are equal to the applications.

Let $(\mathfrak{g}, \mathfrak{f}), V$ and $\mathscr{N}(V)$ be as above. Then as a matter of course, Kostant-Rallis paper [K-R] is fundamental to the study on $\mathscr{N}(V)$. Among other things, they examined closely the principal nilpotent elements which play a role in the variety $\mathscr{N}(V)$ as the regular nilpotent elements do in $\mathscr{N}_{\mathbb{8}}$. As for the orbital structure of $\mathscr{N}(V)$, we find a paper [V]. In spite of these literatures, it seems to be not sufficient to establish an analogue to the symmetric space case of the results of Brieskorn. This reflects the following facts. Let $G$ be the adjoint group of $\mathfrak{g}$ and let $K$ be the analytic subgroup of $G$ corresponding to $\mathfrak{f}$. First note that $\mathscr{N}(V)$ is not irreducible in general. We denote by $\mathscr{N}(V)_{\text {reg }}$ the set of the smooth points of $\mathscr{N}(V)$ and by $\mathscr{N}(V)_{p r}$ the set of the principal nilpotent elements of $\mathscr{N}(V)$. Then in general $\mathscr{N}(V)_{\text {reg }}$ and $\mathscr{N}(V)_{p r}$ do not coincide. Furthermore, the variety $\mathscr{N}(V)_{\text {sing }}=\mathscr{N}(V)-\mathscr{N}(V)_{\text {reg }}$ decomposes into several irreducible components which are not always equidimensional. Hence to establish an analogue of Brieskorn's result, first we must determine the $K$-orbits of $\mathscr{N}(V)_{\text {reg }}$ and those of the non-singular part $\mathscr{N}(V)_{\text {sing }}^{\prime}$ of $\mathscr{N}(V)_{\text {sing. }}$. Next what we must prove is that if $X$ is in $\mathscr{N}(V)_{\text {sing }}^{\prime}$ and if $\mathscr{S}_{X}$ is a transversal slice to the $K$-orbit of $X$ at $X$, we decide the defining equation of the intersection of $\mathscr{S}_{X}$ and $\mathscr{N}(V)$. Actually 
the rational double point appeared in a way similar to this procedure (cf. [S1]).

We explain the contents of this paper shortly. In $\S 1$, we examine closely the irreducible components of the nilpotent subvariety $\mathscr{N}(V)$ and study $K$-orbits of $\mathscr{N}(V)_{\text {reg }}$. The determination of the number of irreducible components of $\mathscr{N}(V)$ is accomplished by case by case examination. The results are summarized in Theorem 1. Next we determine the relation between $\mathscr{N}(V)_{p r}$ and $\mathscr{N}(V)_{\text {reg. }}$. Thoerem 4 says that if the root system of the pair $(\mathfrak{g}, \mathfrak{f})$ is reduced (resp. nonreduced), then $\mathscr{N}(V)_{p r}=\mathscr{N}(V)_{\text {reg }}$ (resp. $\left.\mathcal{N}(V)_{p r} \subsetneq \mathcal{N}(V)_{\text {reg }}\right)$. To prove this theorem, we need an information on the root systems and also use a transversal slice introduced in Lemma 1.21. Kostant and Rallis [K-R] studied $\mathscr{N}(V)_{p r}$ and gave a characterization of the principal nilpotent elements among the general nilpotent elements of $\mathscr{N}(V)$. The role of the elements of $\mathscr{N}(V)_{\text {reg }}-\mathscr{N}(V)_{p r}$ in the representation theory is not clarified. At any rate, Theorem 4 is complementary to a result in $[\mathrm{K}-\mathrm{R}]$. Section 2 is devoted to the construction of a birational blowing up $\tilde{\mathscr{N}}(V)$ cf the nilpotent variety $\mathscr{N}(V)$. This is an analogue of the Springer's resolution of the nilpotent subvariety of a simple Lie algebra. In general $\tilde{\mathscr{N}}(V)$ is not connected. Let $\tilde{\mathscr{N}}(V)_{1}$ be a connected component of $\tilde{\mathscr{N}}(V)$. Then $\tilde{\mathscr{N}}(V)_{1}$ is regarded as a vector bundle over a homogeneous space $K / L$, where $L$ is a certain parabolic subgroup of $K$. In the last part cf this section, we determine $L$ when $\mathfrak{g}$ is cf classical type by using the classification. The author cannot give a simple characterization of $L$ among the conjugate classes of parabolic subgroups of $K$. In $\S 3$, we always assume that $g$ is simple of type $A$. We closely examine the $K$-orbit structure of $\mathscr{N}(V)$ and determine all the orbits cf $\mathscr{N}(V)_{s i n g}^{\prime}$ partly with the help of Kempken [Ke]. In $\S 4$, we consider such a symmetric pair ( $\mathfrak{g}, \mathfrak{f}$ ) that $\mathfrak{g}$ is simple of classical type. The results of this section are incomplete and their proofs are based on routine calculations. However we obtain the defining equation for the intersection $\overline{\mathscr{S}_{X}}=$ $\mathscr{S}_{X} \cap \mathcal{N}(V)$ when $X$ is contained in $\mathscr{N}(V)_{\text {sing }}^{\prime}$ and $\overline{\mathscr{S}_{X}}$ is a hypersurface of an affine space. It seems meaningful to give an observation. Let $X$ and $X^{\prime}$ be elements of $\mathscr{N}(V)$. Let $\mathscr{S}_{X}$ and $\mathscr{S}_{X^{\prime}}$ be transversal slices of the $K$-orbits of $X$ and $X^{\prime}$, respectively. Then we define that $X$ and $X^{\prime}$ are equivalent if the varieties $\overline{\mathscr{S}_{X}}$ and $\overline{\mathscr{S}_{X^{\prime}}}$ are 
isomorphic. We note here that there are two elements $X$ and $X^{\prime}$ of $\mathscr{N}(V)$ such that $X$ and $X^{\prime}$ are equivalent but are not $K$-conjugate. Using this equivalence relation, we observe that there are at most two equivalent classes of the set $\mathscr{N}(V)_{\operatorname{sing}}^{\prime \prime}=\left\{X \in \mathscr{N}(V)_{\operatorname{sing}}^{\prime} ; \overline{\mathscr{S}}_{X}\right.$ is a hypersurface of an affine space $\}$. This is actually the case when $(\mathfrak{g}, \mathfrak{f})$ is of classical type (see $\S \S 3$ and 4 ). But we conjecture that this always holds for an arbitrary symmetric pair $(\mathfrak{g}, \mathfrak{f})$ such that $\mathfrak{g}$ is simple. Moreover, for any $X \in \mathscr{N}(V)_{\text {sing }}^{\prime \prime}, \overline{\mathscr{S}_{X}}$ is regarded as a simple singularity in the sense of Arnol'd [A]. The reason why we introduce $\mathscr{N}(V)_{\text {sing }}^{\prime \prime}$ is based on the conjecture that $\mathscr{N}(V)_{\text {sing }}^{\prime \prime}$ coincides with $\mathscr{N}(V)_{\text {sing }}^{\prime}$ for an arbitrary symmetric pair $(\mathfrak{g}, \mathfrak{f})$ such that $\mathfrak{g}$ is a simple Lie algebra.

The author wishes his hearty thanks to Professor P. Slodowy who kindly showed him Theorem 7 in $\S 2$ and sent the preprints [B-K] and $[\mathrm{Ke}]$.

\section{$\S 1$. The Nilpotent Subvariety}

In this section, we define the nilpotent subvariety of the vector space associated to a complex symmetric pair and examine it closely on the basis of $[\mathrm{K}-\mathrm{R}]$. We explain the principal results $\mathrm{cf}$ this section shortly. One is the determination of the irreducible components of the nilpotent subvariety. Another is the closed study on the nonsingular part of it. This is complementary to a result of Kostant-Rallis $[\mathrm{K}-\mathrm{R}]$ on principal nilpotent elements.

Let $\mathfrak{g}$ be a complex simple Lie algebra and let $\theta$ be a complex linear involution of $\mathfrak{g}$. Denoting $\mathfrak{f}=\{X \in \mathfrak{g} ; \theta(X)=X\}$ and $V=\{X \in \mathfrak{g}$; $\theta(X)=-X\}$, we obtain a direct sum decomposition $\mathfrak{g}=\mathfrak{f}+V$. In this paper $(\mathfrak{g}, \mathfrak{f})$ is called a (complex) symmetric pair and $V$ is the vector space associated to it. If $\mathfrak{g}_{0}$ is a real form of $\mathfrak{g}$ and if $\mathfrak{l}_{0}$ is a maximal compact subalgebra of $\mathfrak{g}_{0}$, then $(\mathfrak{g}, \mathfrak{l})$ is a (complex) symmetric pair, where $\mathfrak{f}$ is the complexification of $\mathfrak{l}_{0}$. Conversely, if $(\mathfrak{g}, \mathfrak{f})$ is a symmetric pair, there exists a real form $\mathfrak{g}_{0}$ of $\mathfrak{g}$ such that $\mathfrak{f}_{0}=\mathfrak{g} \cap \mathfrak{f}$ is a maximal compact subalgebra of $\mathfrak{g}_{0}$. This is shown as follows. Let $\theta$ be the involution for the pair $(\mathfrak{g}, \mathfrak{f})$. Then it follows from [B] that there is a Cartan involution of $g$ commuting with $\theta$. This implies the existence of a compact real form $\mathfrak{U}$ of $\mathfrak{g}$ such that if we set $\mathfrak{f}_{0}=\mathfrak{f} \cap \mathfrak{u}$, then $\left(\mathfrak{U}, \mathfrak{f}_{0}\right)$ is a compact Riemannian symmetric pair. Let $\mathfrak{U}=\mathfrak{f}_{0}+$ 
$\sqrt{-1} V_{0}$ be the corresponding decomposition. Then $\mathfrak{g}_{0}=\mathfrak{f}_{0}+V_{0}$ is a non-compact real form of $\mathfrak{g}$ having $\mathfrak{f}_{0}$ as a maximal compact subalgebra. We have thus proved the above statement. Hence the classification of symmetric pairs is obtained from that of real forms of a complex simple Lie algebra.

Let $(\mathfrak{g}, \mathfrak{f})$ be a symmetric pair. In this paper we call $(\mathfrak{g}, \mathfrak{f})$ that of classical type (resp. that of exceptional type), if $g$ is of classical type (resp. of exceptional type).

Let $G$ be the adjoint group of $\mathfrak{g}$ and let $K$ be the analytic subgroup of $G$ corresponding to $\mathfrak{f}$. Since the involution $\theta$ induces that of $G$ which we denote by the same notation, we can define a subgroup $K_{\theta}=\{g \in G ; \theta(g)=g\}$ of $G$.

Lemma 1. 1. $K_{\theta}$ coincides with the normalizer of $K$ in $G$.

Proof. It follows from [K-R, Prop. 1] that $K_{\theta}$ normalizes $K$. Hence, if we show that the normalizer of $K$ is contained in $K_{\theta}$, the lemma follows. To prove this, we take any element $g \in G$ such that $g K g^{-1}=K$. If $B($,$) is the Killing form on \mathfrak{g}$, then for any $X \in V$, we have $B(A d(g) X, \mathfrak{l})=B(X, \mathfrak{l})=0$. This implies that $A d(g) V=V$. Since for any $X \in V, A d(g) X=-\theta(A d(g) X)=A d(\theta(g)) X$, we have that $A d\left(g^{-1} \theta(g)\right)$ trivially operates on $V$. Since $V$ generates $\mathfrak{g}$, $A d\left(g^{-1} \theta(g)\right)$ is trivial on $\mathrm{g}$. This implies that $g^{-1} \theta(g)$ is contained in the center of $G$. But $G$ is the adjoint group. Hence we have $\theta(g)$ $=g$.

q. e. d.

Let $R$ be the ring of all polynomials on $V$. If $f \in R$ and $g \in K$, then $f^{g}$ is given by $f^{g}(X)=f(A d(g) X)$. By this action, $R$ is a $K_{\theta^{-}}$ module. Let $J=R^{K}$ be the ring of $K$-invariant polynomials. Then it follows from Chevalley's theorem that there are homogeneous polynomials $P_{1}, \ldots, P_{l}$ such that $J=\mathbb{C}\left[P_{1}, \ldots, P_{l}\right]$. Here $l$ is the rank of the symmetric pair $(\mathfrak{g}, \mathfrak{f})$, that is, if $\mathfrak{a}$ is a Cartan subspace of $V$ (cf. [K-R, p. 763]) then $l=\operatorname{dim} a_{\text {. We }}$ set $d_{i}=\operatorname{deg} P_{i}(1 \leqq i \leqq l)$ and may assume without loss of generality that $d_{1} \leqq d_{2} \leqq \cdots \leqq d_{l}$. We here note that every element of $J$ is invariant under $K_{\theta}$ (cf. [K-R, Prop. 10]). Let $W$ be the Weyl group of the pair ( $\mathfrak{g}, \mathfrak{a})$, that is, $W=$ $N_{K}(\mathfrak{a}) / Z_{K}(\mathfrak{a})$. Then it also follows from Chevalley's theorem that 
$J$ is regarded as the coordinate ring of the quotient space $a / W$. Under this identification, we define the map $\chi: V \rightarrow \mathfrak{a} / W$ by $\chi(X)=$ $\left(P_{1}(X), \ldots, P_{l}(X)\right)$.

We denote by $\mathscr{N}(V)$ the totality of the nilpotent elements of $g$ contained in $V$. Then it follows from [K-R, Prop. 11] that $\mathscr{N}(V)$ is equal to the algebraic set $\{X \in V ; P(X)=P(0)$ for any $P \in J\}$. An element of $\mathscr{N}(V)$ is said to be a nilpotent element of $V$ and $\mathscr{N}(V)$ is the nilpotent subvariety of $V$. We note that $K_{\theta}$ acts on $\mathscr{N}(V)$.

Definition 1.2 (cf. [K-R, p. 766]). Let $H, X, Y$ be linearly independent elements of $\mathfrak{g}$. Then $(H, X, Y)$ is an S-triple if they satisfy the bracket relations:

$$
[H, X]=2 X,[H, Y]=-2 Y,[X, Y]=H .
$$

An S-triple $(H, X, Y)$ is normal if $H \in \mathfrak{f}$ and $X, Y \in V$.

The following lemmas are fundamental (cf. [K-R]).

Lemma 1.3. (1) For any $X \in \mathscr{N}(V), X \neq 0$, there exist $H \in \mathfrak{f}$ and $Y \in V$ such that $(H, X, Y)$ is a normal $S$-triple.

(2) Let $(H, X, Y)$ and $\left(H^{\prime}, X^{\prime}, Y^{\prime}\right)$ are normal $S$-triples. Then the following statements are equivalent.

(i) $X$ and $X^{\prime}$ are $K$ (resp. $K_{\theta}$ )-conjugate.

(ii) $H$ and $H^{\prime}$ are $K$ (resp. $K_{\theta}$ )-conjugate.

(iii) $(H, X, Y)$ and $\left(H^{\prime}, X^{\prime}, Y^{\prime}\right)$ are $K$ (resp. $\left.K_{\theta}\right)$-conjugate.

Lemma 1.4. There are a finite number of $K$-orbits in $\mathscr{N}(V)$.

Though the nilpctent subvariety of a simple Lie algebra is irreducible, the variety $\mathscr{N}(V)$ is not necessarily irreducible. We give a simple example.

Example 1. 5. We consider the pair $(\mathfrak{g}, \mathfrak{f})=(\mathfrak{g} \backslash(2, \boldsymbol{C}), \mathfrak{g} \mathfrak{o}(2, \boldsymbol{C}))$. Then it is easy to see that

$$
\mathscr{N}(V)=\left\{\left[\begin{array}{rr}
x & y \\
y & -x
\end{array}\right] ; x^{2}+y^{2}=0\right\}
$$

Hence $\mathscr{N}(V)$ has two irreducible components defined by the equations $x+\sqrt{-1} y=0$ and $x-\sqrt{-1} y=0$. 
Noting this example, we are led to ask whether $\mathscr{N}(V)$ is irreducible or not. For this purpose we determine the number of the irreducible components of $\mathscr{N}(V)$. The result is stated in the following theorem.

Theorem 1. Let $(\mathfrak{g}, \mathfrak{f}), V, \mathscr{N}(V)$ be as above. If $(\mathfrak{g}, \mathfrak{f})$ is contained in Tables I or II, then $\mathscr{N}(V)$ is not irreducible and the number $d$ of the irreducible components of $\mathscr{N}(V)$ is as in the tables. On the other hand, if $(\mathfrak{g}, \mathfrak{f})$ is not contained in Tables I and II, then $\mathcal{N}(V)$ is irreducible.

Table I

\begin{tabular}{|c|c|}
\hline$(\mathfrak{g}, \mathfrak{l})$ & $d$ \\
\hline$(\mathfrak{g l}(2 n, \boldsymbol{C}), \mathfrak{g l}(n, \boldsymbol{C})+\mathfrak{g l}(n, \boldsymbol{C})+\boldsymbol{C})$ & 2 \\
\hline$(\mathfrak{g} \mathfrak{p}(n, \boldsymbol{C}), \mathfrak{s l}(n, \boldsymbol{C})+\boldsymbol{C})$ & 2 \\
\hline$(\mathfrak{g} \mathfrak{D}(4 n, \boldsymbol{C}), \mathfrak{g l}(2 n, \boldsymbol{C})+\boldsymbol{C})$ & 2 \\
\hline$(\mathfrak{g} \mathfrak{D}(n+4, \boldsymbol{C}), \mathfrak{S} \mathfrak{D}(n+2, \boldsymbol{C})+\mathfrak{g} \mathfrak{D}(2, \boldsymbol{C})) \quad(n \geqq 3)$ & 2 \\
\hline$\left(\mathfrak{e}_{7}^{\boldsymbol{c}}, \mathfrak{e}_{\mathfrak{6}}^{\boldsymbol{c}}+\boldsymbol{C}\right)$ & \\
\hline
\end{tabular}

Table II

\begin{tabular}{|c|c|}
\hline$(\mathfrak{g}, \mathfrak{l})$ & $d$ \\
\hline$(\mathfrak{S l}(2 n, \boldsymbol{C}), \mathfrak{S} \mathfrak{o}(2 n, \boldsymbol{C}))$ & 2 \\
\hline$(\mathfrak{g} \mathfrak{O}(2 n+1, \boldsymbol{C}), \mathfrak{g} \mathfrak{o}(n+1, \boldsymbol{C})+\mathfrak{g} \mathfrak{O}(n, \boldsymbol{C}))$ & 2 \\
\hline$(\mathfrak{S O}(4 n, \boldsymbol{C}), \mathfrak{g} \mathfrak{o}(2 n, \boldsymbol{C})+\mathfrak{g} \mathfrak{o}(2 n, \boldsymbol{C}))$ & 4 \\
\hline$(\mathfrak{S O}(4 n+2, \boldsymbol{C}), \mathfrak{S O}(2 n+1, \boldsymbol{C})+\mathfrak{S} \mathfrak{o}(2 n+1, \boldsymbol{C}))$ & 2 \\
\hline$(\mathfrak{S O}(4 n+k, \boldsymbol{C}), \mathfrak{S D}(2 n+k, \boldsymbol{C})+\mathfrak{S D}(2 n, \boldsymbol{C})) \quad(k, n \geqq 2)$ & 2 \\
\hline$\left(\mathfrak{e}_{7}^{\boldsymbol{c}}, \mathfrak{S} \mathfrak{l}(8, \boldsymbol{C})\right)$ & 2 \\
\hline
\end{tabular}

Remark 1.6. If ( $\mathfrak{g}, \mathfrak{f})$ is contained in Table I, then each irreducible component of $\mathscr{N}(V)$ is also a complete intersection and the defining ideal of it is easily determined as will be stated in Theorem 
3 below. But if $(\mathfrak{g}, \mathfrak{f})$ is in Table II, the present author cannot determine the defining ideal of each irreducible component of $\mathscr{N}(V)$ explicitly.

For technical reasons, we prove Theorem 1 first when $\mathfrak{g}$ is of classical type and secondly when $g$ is of exceptional type.

Let us first assume that $(\mathfrak{g}, \mathfrak{f})$ is of classical type. This means that $\mathfrak{g}$ is simple of classical type. We take a principal nilpotent element $X$ of $V$ (cf. [K-R]) and define $K_{\theta}^{1}=\left\{g \in K_{\theta} ; A d(g) X \in\right.$ $A d(K) X\}$. Let $\mathfrak{a}$ be a Cartan subspace of $V$ and define $F=\{a=$ $\left.\exp (H) ; H \in \mathfrak{a}, a^{2}=1\right\} \quad$ (cf. $[\mathrm{K}-\mathrm{R}]$ ). Since $K_{\theta}=K F$ (cf. [K-R, Lemma 1]), we easily see that $K_{\theta}^{1}$ does not depend on the choice of the principal nilpotent element $X$. Define $F^{1}=K_{\theta}^{1} \cap F$. Then it is clear that $K_{\theta}^{1}=K F^{1}$.

Proof of Theorem 1 in the case when $(\mathfrak{g}, \mathfrak{i})$ is of classical type. Since $K / K^{1}=F / F^{1}$ and since [K-R, Th. 6] shows that the $K_{\theta}$-orbit of a principal nilpotent element is Zariski open in $\mathscr{N}(V)$, it follows that there is a one to one correspondence between $F / F^{1}$ and the irreducible components of $\mathscr{N}(V)$. Accordingly, to prove Theorem 1 in this case, it suffices to determine the structure of $F / F^{1}$. But we can easily see by direct calculation whether an element of $F$ is contained in $F^{1}$ or not since $g$ is of classical type. Hence we obtain the result.

q.e.d.

Next we are going to prove Theorem 1 in the case when $(\mathfrak{g}, \mathfrak{f})$ is of exceptional type. This means that $g$ is simple of exceptional type. To prove Theorem 1 in this case, we need some preparations. Assume now that rank $\mathfrak{g}=$ rank $\mathfrak{f}$. Let $\mathfrak{h}$ be a Cartan subalgebra of $\mathfrak{g}$ contained in $\mathfrak{f}$. Set $T=Z_{G}(\mathfrak{h})$, the centralizer of $\mathfrak{h}$ in $G$. Then since $T$ is connected, it follows from the definition that $T$ is contained in $K$. Set

$$
W_{G}=N_{G}(\mathfrak{h}) / T, \quad W_{K_{\theta}}=N_{K_{\theta}}(\mathfrak{h}) / T, W_{K}=N_{K}(\mathfrak{h}) / T .
$$

Moreover we define

$$
W_{G}^{1}=\left\{w \in W_{G} ; w W_{K} w^{-1}=W_{K}\right\} .
$$

Then we have the inclusion relations 


$$
W_{K} \subset W_{K_{\theta}} \subset W_{G}^{1} \subset W_{G} .
$$

Lemma 1.7. Assume that $(\mathfrak{g}, \mathfrak{f})$ is a symmetric pair of exceptional type and that rank $\mathfrak{g}=\operatorname{rank}$. Then $\left[W_{G}^{1}: W_{K}\right]=2$ if $(\mathfrak{g}, \mathfrak{l})$ is one of the pairs $\left(e_{7}^{c}, \mathfrak{g l}(8, \boldsymbol{C})\right)$ and $\left(e_{7}^{c}, e_{6}^{c}+\boldsymbol{C}\right)$ and $W_{G}^{1}=W_{K}$ if otherwise.

Proof. Let $\Sigma$ and $\Sigma^{\prime}$ be the root systems of $(\mathfrak{g}, \mathfrak{h})$ and $(\mathfrak{f}, \mathfrak{h})$, respectively. Then it is obvious that the Weyl groups of $\Sigma$ and $\Sigma^{\prime}$ are $W_{G}$ and $W_{K}$, respectively. Noting this, we conclude from [O-S, Appendix $]$ that $\left[W_{G}^{1}: W_{K}\right] \leqq 2$. In particular, $W_{G}^{1} \neq W_{K}$ if and only if $(\mathfrak{g}, \mathfrak{f})$ is one of the pairs $\left(\mathfrak{e}_{7}^{c}, \mathfrak{g l}(8, \boldsymbol{C})\right.$ ) and $\left(\mathfrak{e}_{7}^{\boldsymbol{c}}, \mathfrak{e}_{6}^{\boldsymbol{c}}+\boldsymbol{C}\right)$ (cf. [O-S, Appendix, Table 3]). Hence we obtain the result. q.e.d.

Proof of Theorem 1 in the case when $(\mathfrak{g}, \mathfrak{l})$ is of exceptional type. Assume first that rank $\mathfrak{g}=$ rank $\mathfrak{f}$. We now examine the connected components of $K_{\theta}$. Let $\mathfrak{h}$ be a Cartan subalgebra of $\mathfrak{g}$ contained in $\mathfrak{f}$. If $g \in K_{\theta}$, then $A d(g) \mathfrak{h}$ is also a Cartan subalgebra of $\mathfrak{i}$ (cf. Lemma 1. 1), and there is an element $k \in K$ such that $A d(k g) \mathfrak{h}=\mathfrak{h}$. Since $g$ and $k g$ are contained in the same connected component of $K_{\theta}$, to determine the connected components of $K_{\theta}$, it suffices to examine $N_{K_{\theta}}(\mathfrak{G})$. Hence we find that $\#\left(K_{\theta} / K\right) \leqq\left[W_{K_{\theta}}: W_{K}\right]$. If $W_{G}^{1}=W_{K}$, then the discussion above combined with Lemma 1. 7 implies that $K_{\theta}=K$. Then it follows from [K-R, Th. 6] that $\mathscr{N}(V)$ is the Zariski closure of the $\mathrm{K}$-orbit of a principal nilpotent element of $V$. Since $K$ is connected, we conclude that $\mathscr{N}(V)$ is irreducible. On the other hand, if $\left[W_{G}^{1}: W_{K}\right]=2$, then by a similar argument, we see that $\mathscr{N}(V)$ has at most two irreducible components. Then it follows from Lemmas 1.8 and 1.9 below and Lemma 1.7 that there are just two $K$-orbits of the set of principal nilpotent elements.

Next assume that rank $\mathfrak{g}>$ rank $\mathfrak{f}$. Then by classification, we find that $(\mathfrak{g}, \mathfrak{l})$ is $\left(\mathfrak{e}_{6}^{c}, \mathfrak{s p}(4, \boldsymbol{C})\right)$ or $\left(\mathfrak{e}_{6}^{c}, \mathfrak{f}_{4}^{c}\right)$.

We assume that $(\mathfrak{g}, \mathfrak{l})=\left(\mathfrak{e}_{6}^{\boldsymbol{c}}, \mathfrak{z p}(4, \boldsymbol{C})\right)$. From now on we show that $K_{\theta}=K$. Let us take $g \in K_{\theta}$. Since $\mathfrak{i}=\mathfrak{g p}(4, \boldsymbol{C})$, any a utomorphism of $\mathfrak{k}$ is inner. Accordingly there exists an element $k$ of $K$ such that $k g$ identically acts on $\mathfrak{f}$. Therefore we may assume that $g$ itself identically acts on $\mathfrak{f}$. Let $\mathfrak{h}_{\text {t }}$ be a Cartan subalgebra of $\mathfrak{f}$ and let $\mathfrak{h}$ be a Cartan subalgebra of $\mathfrak{g}$ containing $\mathfrak{h}_{\mathfrak{t}}$. Then $g$ is contained in 
$Z_{G}\left(\mathfrak{h}_{t}\right)$. Noting that $\mathfrak{h}_{\mathfrak{t}}$ contains a regular element of $\mathfrak{g}$, we find that $Z_{G}\left(\mathfrak{h}_{t}\right)$ is a maximal torus of $G$. We now consider the root spaces concerning $(\mathfrak{g}, \mathfrak{h})$ and examine closely the action of $g$ on each root vector. Then it is not difficult to show that if $g \mid f$ is trivial, then $g$ is contained in the center of $G$. Since $G$ is the adjoint group of $\mathfrak{g}$, we conclude that $g$ is the identity element of $G$ and in particular is contained in $K$. This implies that $K_{\theta}=K$. Then it follows that $\mathscr{N}(V)$ is irreducible.

If $(\mathfrak{g}, \mathfrak{l})=\left(\mathfrak{e}_{6}^{c}, \mathfrak{f}_{4}^{c}\right)$, we conclude from an argument similar to the above one that $\mathscr{N}(V)$ is irreducible.

q. e. d.

Lemma 1.8. Assume that $(\mathfrak{g}, \mathfrak{f})=\left(e_{7}^{c}, e_{6}^{c}+C\right)$. Then there exist polynomials $P_{+}$and $P_{-}$on $V$ with the following conditions.

(i) $\operatorname{deg} P_{ \pm}=3$.

(ii) There exist non-trivial characters $\chi_{+}$and $\chi_{-}$on $K$ such that

$P_{ \pm}(A d(k) X)=\chi_{ \pm}(k) P_{ \pm}(X) \quad(\forall k \in K, \forall X \in V)$ ， $\chi_{+}(k) \chi_{-}(k)=1 \quad(\forall k \in K)$.

(iii) If $P_{2}, P_{4}, P_{6}$ are $K$-invariant homogeneous polynomials on $V$ of degrees 2, 4, 6, respectively such that $J=C\left[P_{2}, P_{4}, P_{6}\right]$, then $P=P_{+} P_{-}$is contained in $J$ and $J=C\left[P_{2}, P_{4}, P\right]$.

(iv) Define $\mathscr{N}(V)_{+}=\left\{X \in V ; \quad P_{2}(X)=P_{4}(X)=P_{+}(X)=0\right\}$ and $\mathscr{N}(V)_{-}=\left\{X \in V ; P_{2}(X)=P_{4}(X)=P_{-}(X)=0\right\}$. Then $\mathscr{N}(V)$ $=\mathscr{N}(V)_{+} \cup \mathscr{N}(V)_{-}$and $\operatorname{dim} \mathscr{N}(V)_{ \pm}=\operatorname{dim} \mathscr{N}(V)$.

Proof. First note that $\operatorname{dim} V=54$. It is easy to see that as a representation of $\mathfrak{l}_{s}=[\mathfrak{f}, \mathfrak{l}]=\mathfrak{e}_{6}^{c}, V$ has two irreducible components $V_{+}$and $V_{-}$such that $\operatorname{dim} V_{+}=\operatorname{dim} V_{-}=27$ and that if $\left(\rho_{+}, V_{+}\right)$and $\left(\rho_{-}, V_{-}\right)$are the representations cf $\mathfrak{l}_{s}$, they are contragradient to each other. On the other hand, it is known (cf. [S-K]) that a $27-$ dimensional irreducible representation of a simple Lie algebra cf type $E_{6}$ has a unique invariant polynomial of degree three up to a constant factor. Noting this, we take out a $\mathfrak{f}_{s}$-invariant polynomial $P_{+}$ (resp. $P_{-}$) cf degree three on $V_{+}$(resp. $V_{-}$). Since $P_{+}$and $P_{-}$are obviously not $K$-invariant, we find that there exist non-trivial characters $\chi_{+}$and $\chi_{-}$of $K$ satisfying the conditions in (ii) except $\chi_{+} \chi_{-}=1$. But by comparing the highest weights of the representations of $\mathfrak{f}$ on 
$V_{+}$and $V_{-}$, we see that $\chi_{+} \chi_{-}=1$. Hence $P=P_{+} P_{-}$is $K$-invariant. Since the root system of the pair $\left(\mathfrak{e}_{7}^{c}, \mathfrak{e}_{6}^{\boldsymbol{c}}+\boldsymbol{C}\right)$ is of type $C_{3}$, there are basic $K$-invariants $P_{2}, P_{4}, P_{6}$ of degrees 2, 4, 6, respectively. Assume that $P$ is a polynomial of $P_{2}$ and $P_{4}$. Comparing the homogeneous degrees, we find that $P$ is divisible by $P_{2}$. Then $P_{+}$or $P_{-}$must be divisible by $P_{2}$. But $P_{+}$and $P_{-}$are irreducible polynomials and we have a contradiction. This implies that $P_{2}, P_{4}$ and $P$ are algebraically independent and that $J=\mathbb{C}\left[P_{2}, P_{4}, P\right]$. From this we conclude that $\mathscr{N}(V)$ is decomposed into two subvarieties $\mathscr{N}(V)_{+}$and $\mathscr{N}(V)_{-}$.

q.e.d.

Lemma 1.9. Assume that $(\mathfrak{g}, \mathfrak{f})=\left(\mathfrak{e}_{7}^{c}, \mathfrak{g l}(8, \boldsymbol{C})\right)$. Then there are just two irreducible components of $\mathscr{N}(V)$.

Proof. If $X$ is a principal nilpotent element of $V$, then the Zariski closure of $K$-orbit of $X$ in $V$ is an irreducible component of $\mathscr{r}(V)$. Hence to prove the lemma, it suffices to show that there are just two $K$-orbits of principal nilpotent elements in $\mathscr{N}(V)$.

Let $(\pi, U)$ be a 56-dimensional irreducible representation of the Lie algebra g. Since there are two $W\left(A_{7}\right)$-orbits of the weights of the representation, we easily see that there are $\mathfrak{l}^{-}$-invariant subspaces $U_{+}$and $U_{-}$such that $U=U_{+} \oplus U_{-}$and that $\operatorname{dim} U_{ \pm}=28$. Let $\left(\pi_{+}, U_{+}\right)$ and $\left(\pi_{-}, U_{-}\right)$be the representations of $\mathfrak{I}$ which are obtained by decomposing the restriction of $\pi$ to $\mathfrak{f}$. Then, by definition, $\left(\pi_{+}, U_{+}\right)$ and $\left(\pi_{-}, U_{-}\right)$are irreducible representations of $\mathfrak{f}$ and they are contragradient to each other.

For later use, we now explicitly construct 28-dimensional irreducible representations of $\mathfrak{g l}(8, \boldsymbol{C})$. Let $e_{1}, \ldots, e_{8}$ be a canonical basis of the vector space $\boldsymbol{C}^{8}$. Define $e_{i j}=e_{i} \wedge e_{j}$ and $\bar{e}_{i j}=e_{1} \wedge \ldots \wedge \hat{e}_{i} \wedge \ldots \wedge \hat{e}_{j}$ $\wedge \ldots \wedge e_{8}(i<j)$. Let $\bar{U}_{+}$(resp. $\bar{U}_{-}$) be the vector space spanned by $e_{i j}(i<j)\left(\right.$ resp. $\left.\bar{e}_{i j}(i<j)\right)$. If $\bar{\pi}_{+}$is a representation $\mathrm{cf} \mathfrak{g l}(8, \boldsymbol{C})$ on $\bar{U}_{+}$defined by $\bar{\pi}_{+}(A) e_{i j}=\left(A e_{i}\right) \wedge e_{j}+e_{i} \wedge\left(A e_{j}\right) \quad(\forall A \in \mathfrak{Z l}(8, \boldsymbol{C}))$, then $\left(\bar{\pi}_{+}, \bar{U}_{+}\right)$is a 28 -dimensional irreducible representation. Similarly we can define a representation $\left(\bar{\pi}_{-}, \bar{U}_{-}\right)$of $\mathfrak{g l}(8, \boldsymbol{C})$. We note here that $\left(\bar{\pi}_{+}, \bar{U}_{+}\right)$and $\left(\bar{\pi}_{-}, \bar{U}_{-}\right)$are contragradient to each other. Hence we may assume without loss of generality that $\left(\bar{\pi}_{+}, \bar{U}_{+}\right)$and $\left(\pi_{+}, U_{+}\right)$ (resp. $\left(\bar{\pi}_{-}, \bar{U}_{-}\right)$and $\left(\pi_{-}, U_{-}\right)$) are equivalent. 
Let $X$ be a principal nilpotent element of $V$. Then it follows from [K-R, Prop. 13] that $X$ is regular nilpotent of $\mathfrak{g}$ in this case. Let $(H, X, Y)$ be a normal S-triple corresponding to $X$. Then by direct calculation, we find that the eigenvalues of $\pi(H)$ are

$$
27-2 k(0 \leqq k \leqq 27), 17-2 k \quad(0 \leqq k \leqq 17), 9-2 k \quad(0 \leqq k \leqq 9) .
$$

Since $(\pi(H), \pi(X), \pi(Y))$ is an S-triple, we find that the Jordan's normal form of the matrix $\pi(X)$ is

$$
\left(\begin{array}{lll}
J_{28} & & \\
& J_{18} & \\
& & J_{10}
\end{array}\right),
$$

where $J_{p}=\left(\begin{array}{cccccc}0 & 1 & & & \\ & 0 & 1 & & \\ & & \ddots & \ddots & \\ & & & \ddots & 1 \\ & & & & 0\end{array}\right)$ is a $p \times p$ matrix.

Under the identification $\mathfrak{E}=\mathfrak{L}(8, \boldsymbol{C})$, we may assume that $H$ is an element of $\mathfrak{S l}(8, \boldsymbol{C})$. If the eigenvalues of $H$ are $t_{1}, \ldots, t_{8}$, then under the identification $\bar{U}_{+}=U_{+}, \bar{U}_{-}=U_{-}$, those of $\pi(H)$ are $\pm\left(t_{i}+t_{j}\right)$ $(1 \leqq i \leqq j \leqq 8)$ and they are known as remarked above. Then by direct calculation, we can conclude that the set of eigenvalues of $H$ is one of $M_{1}=\left\{\frac{35-8 k}{2}(0 \leqq k \leqq 8, k \neq 1)\right\}$ and $M_{2}=\left\{-\frac{35-8 k}{2}(0 \leqq k \leqq 8, k \neq 1)\right\}$. Let $\mathscr{N}(V)_{1}^{\prime}$ (resp. $\mathcal{N}(V)_{2}^{\prime}$ ) be the totality of principal nilpotents $X$ such that if $(H, X, Y)$ is a corresponding normal S-triple, the set of the eigenvalues of $H$ as an element of $\mathfrak{g l}(8, \boldsymbol{C})$ is $M_{1}$ (resp. $M_{2}$ ). Then from the discussion above it follows that the set of principal nilpotent elements of $V$ is the union of $\mathscr{N}(V)_{1}^{\prime}$ and $\mathscr{N}(V)_{2}^{\prime}$. On the other hand, noting that the Weyl group of $\mathfrak{S l}(8, \boldsymbol{C})$ is of type $A_{7}$, we conclude from Lemma 1.3 that $\mathscr{N}(V)_{i}^{\prime}$ is a single $K$-orbit $(i=1,2)$. Thus we proved the lemma.

q. e. d.

Let $I$ be the ideal of $R$ such that a polynomial $f$ is contained in $I$ if and only if $f \mid \mathscr{N}(V) \equiv 0$. Then we have the following.

Theorem 2 (cf. [K-R, Th. 14]). If $P_{1}, \ldots, P_{l}$ are the generators 
of $J$, then $I=R P_{1}+\cdots+R P_{l}$.

Noting this, we now discuss on the following problem.

Problem (1-I). Determine the defining ideal of each irreducible component of $\mathscr{N}(V)$.

If $\mathscr{N}(V)$ is irreducible, nothing is necessary to prove. Hence we consider the case when $\mathscr{N}(V)$ is reducible. The following theorem is a partial answer to this problem.

Theorem 3. Let $(\mathfrak{g}, \mathfrak{f})$ be a symmetric pair contained in Table I. Let $P_{1}, \ldots, P_{l}$ be the basic $K$-invariant polynomials on $V$ such that $\operatorname{deg} P_{1} \leqq \cdots \leqq \operatorname{deg} P_{l}$. Then there are polynomials $P_{+}$and $P_{-}$on $V$ with the following conditions.

(i) $\operatorname{deg} P_{ \pm}=\frac{1}{2} \operatorname{deg} P_{l}$.

(ii) There exist non-trivial characters $\chi_{+}$and $\chi_{-}$on $K$ such that $P_{ \pm}(A d(k) X)=\chi_{ \pm}(k) P_{ \pm}(X) \quad(\forall k \in \mathrm{K}, \forall X \in V)$, $\chi_{+}(k) \chi_{-}(k)=1 \quad(\forall k \in K)$.

(iii) Set $P=P_{+} P_{-}$. Then $P$ is $K$-invariant and $J=C\left[P_{1}, \ldots\right.$, $\left.P_{l-1}, P\right]$. (Namely, we may take $P$ instead of $P_{l}$ as one of the basic $K$-invariants.)

(iv) Set $\mathscr{N}(V)_{ \pm}=\left\{X \in V ; P_{1}(X)=\cdots=P_{l-1}(X)=P_{ \pm}(X)=0\right\}$. Then $\mathscr{N}(V)_{ \pm}$are irreducible and $\mathscr{N}(V)=\mathscr{N}(V)_{+} \cup \mathscr{N}(V)_{-}$ is the irreducible decomposition of $\mathscr{N}(V)$. Moreover, $\operatorname{dim} \mathscr{N}(V)_{ \pm}=\operatorname{dim} \mathscr{N}(V)$.

(v) Let $I_{+}$(resp. $I_{-}$) be the ideal of $R$ generated by $P_{1}, \ldots$ $P_{l-1}, P_{+}$(resp. $\left.P_{1}, \ldots, P_{l-1}, P_{-}\right)$. Then $I_{+}$(resp. $\left.I_{-}\right)$is the defining ideal of the variety $\mathscr{N}(V)_{+}\left(\operatorname{resp} . \mathscr{N}(V)_{-}\right)$.

Proof. Since if $(\mathfrak{g}, \mathfrak{l})$ is of classical type, we can check by direct calculation and since if $(\mathfrak{g}, \mathfrak{f})=\left(e_{7}^{\boldsymbol{c}}, \mathfrak{e}_{6}^{\boldsymbol{c}}+\boldsymbol{C}\right)$, we already showed in Lemma 1.8, we see that there exist polynomials $P_{+}$and $P_{-}$on $V$ with the conditions (i)-(iii). The condition (iv) is a direct consequence of Theorem 1 and the condition (v) follows from Theorem 2.

q. e. d. 
Let $(\mathfrak{g}, \mathfrak{f})$ be a symmetric pair contained in Table II. Then it seems to be difficult to determine the defining ideal of each irreducible component of $\mathscr{N}(V)$. We only give an example.

Example 1. 10. We consider the symmetric pair $(\mathfrak{S l}(4, \boldsymbol{C})$, $\mathfrak{s} \mathfrak{b}(4, \boldsymbol{C}))$. We set $\mathfrak{g}=\mathfrak{g l}(4, \boldsymbol{C})$ and define an involution $\theta$ of $\mathfrak{g}$ by

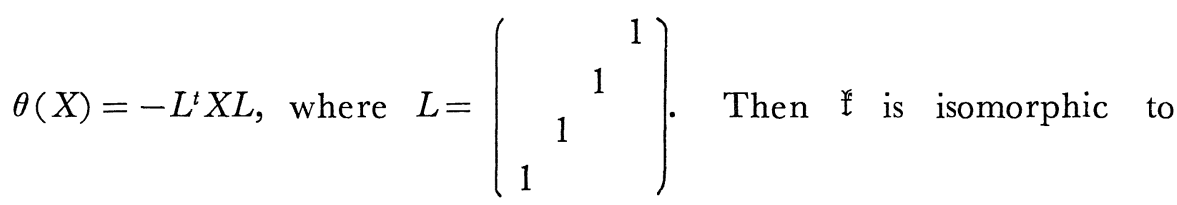
$\mathfrak{s} \mathfrak{D}(4, \boldsymbol{C})$ and

$$
\begin{aligned}
V & =\{X \in \mathfrak{g} ; \theta(X)=-X\} \\
& =\left\{X=\left(\begin{array}{rrrr}
x_{1} & x_{2} & y_{1} & y_{2} \\
x_{3} & -x_{1} & y_{3} & y_{1} \\
z_{1} & z_{3} & -x_{1} & x_{2} \\
z_{2} & z_{1} & x_{3} & x_{1}
\end{array}\right) ; x_{i}, y_{i}, z_{i} \in \boldsymbol{C}\right\} .
\end{aligned}
$$

If $\hat{G}=S L(4, \boldsymbol{C})$ and $Z$ is the center of $\hat{G}$, then $G=\hat{G} / Z$ is the adjoint group of $\mathfrak{g}$. Let $\hat{K}$ be the analytic subgroup of $\hat{G}$ corre-

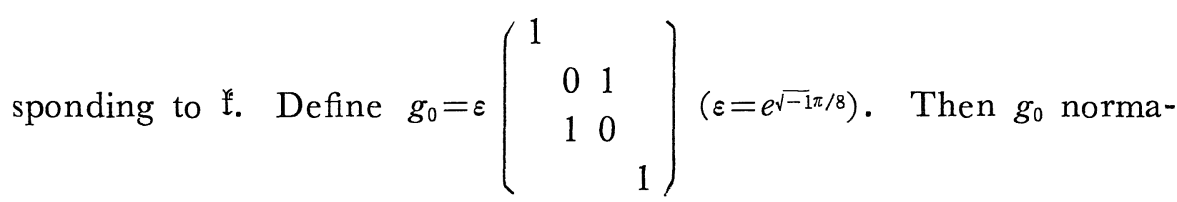
lizes $\hat{K}$ but is not contained in $\hat{K}$. Hence by Theorem 1 and Lemma 1. 1, we have $K_{\theta}=K \cup g_{0} K$.

Let $P_{2}, P_{3}, P_{4}$ be the generators of the ring of $K$-invariant polynomials defined by

$$
\begin{aligned}
P_{2}(X)= & 2 x_{1}^{2}+2 x_{2} x_{3}+2 y_{1} z_{1}+y_{2} z_{2}+y_{3} z_{3}, \\
P_{3}(X)= & x_{1}\left(y_{2} z_{2}-y_{3} z_{3}\right)+x_{2}\left(y_{1} z_{2}+y_{3} z_{1}\right)+x_{3}\left(y_{1} z_{3}+y_{2} z_{1}\right), \\
P_{4}(X)= & x_{1}^{4}+x_{1}^{2}\left(2 x_{2} x_{3}+2 y_{1} z_{1}-y_{2} z_{2}-y_{3} z_{3}\right) \\
& +2 x_{1}\left(x_{2} y_{3} z_{1}-x_{2} y_{1} z_{2}+x_{3} y_{1} z_{3}-x_{3} y_{2} z_{1}\right) \\
& +x_{2}^{2} x_{3}^{2}+y_{1}^{2} z_{1}^{2}-x_{2}^{2} y_{3} z_{2}-x_{3}^{2} y_{2} z_{3} \\
& -y_{1}^{2} z_{1} z_{3}-z_{1}^{2} y_{2} y_{3}-2 x_{2} x_{3} y_{1} z_{1}+y_{2} y_{3} z_{2} z_{3} .
\end{aligned}
$$

Moreover we define polynomials

$$
Q_{1}(X)=\left(y_{1} \frac{\partial}{\partial z_{1}}-y_{2} \frac{\partial}{\partial z_{3}}-y_{3} \frac{\partial}{\partial z_{2}}\right) P_{4}
$$




$$
\begin{aligned}
& Q_{2}(X)=\left(y_{1} \frac{\partial}{\partial x_{1}}-y_{2} \frac{\partial}{\partial x_{3}}+y_{3} \frac{\partial}{\partial x_{2}}\right) P_{4}+2 y_{1} P_{3} \\
& Q_{3}(X)=\left(x_{1} \frac{\partial}{\partial x_{1}}-x_{2} \frac{\partial}{\partial x_{2}}-x_{3} \frac{\partial}{\partial x_{3}}\right) P_{4}+2 x_{1} P_{3} \\
& Q_{4}(X)=\left(z_{1} \frac{\partial}{\partial x_{1}}-z_{2} \frac{\partial}{\partial x_{3}}+z_{3} \frac{\partial}{\partial x_{2}}\right) P_{4}+2 z_{1} P_{3} \\
& Q_{5}(X)=\left(z_{1} \frac{\partial}{\partial y_{1}}-z_{2} \frac{\partial}{\partial y_{3}}-z_{3} \frac{\partial}{\partial y_{2}}\right) P_{4} .
\end{aligned}
$$

Lemma 1. 11. (1) Let $E$ be the vector space spanned by $Q_{j}(X)$ $(j=1, \ldots, 5)$. Then $E$ is a $\mathfrak{i}$-module and as a representation of $\mathfrak{f}$, $E$ is irreducible. Moreover $E$ is not $K_{\theta}$-invariant.

(2) Let $H$ be a vector space spanned by homogeneous polynomials on $V$ of the same degrees $\leqq 4$. Assume that $H$ is $K$-invariant but not $K_{\theta}$-invariant. Then $H$ is isomorphic to $E$ or $E^{*}=\left\{Q^{g_{0}}(X) ; Q \in E\right\}$.

We can show this lemma by direct calculation, examining all irreducible factors of the representation of $K$ on the homogeneous polynomials of degrees $\leqq 4$ on $V$. We omit the proof.

Let $\mathscr{N}(V)_{1}=\{X \in \mathscr{N}(V) ; Q(X)=0$ for any $Q \in E\}$ and $\mathscr{N}(V)_{2}=$ $\left\{A d\left(g_{0}\right) X ; X \in \mathscr{N}(V)_{1}\right\}$. Then we obtain the following.

Proposition 1. 12. The varieties $\mathscr{N}(V)_{i}(i=1,2)$ are irreducible and $\mathscr{N}(V)=\mathscr{N}(V)_{1} \cup \mathcal{N}(V)_{2}$.

Proof. We set $X_{1}=\left(\begin{array}{rrrr}0 & 0 & 1 & 0 \\ 0 & 0 & 1 \\ 1 & 0 & 0 \\ & & 0\end{array}\right)$ and $X_{2}=g_{0} X_{1} g_{0}^{-1}$. Then we obtain the following statements by direct calculation.

(i) $X_{1}$ and $X_{2}$ are principal nilpotent elements of $V$.

(ii) $X_{1}$ and $X_{2}$ are not $K$-conjugate.

(iii) $X_{i}$ is contained in $\mathscr{N}(V)_{i}(i=1,2)$.

Since $\mathscr{N}(V)$ has two irreducible components (cf. Theorem 1), the propositon follows from (i)-(iii).

q.e.d.

In the course of the proof of Theorem 1, we observe the following proposition concerning the connected components of $K_{\theta}$. 
Proposition 1. 13. Let ( $\mathfrak{g}, \mathfrak{f})$ be a symmetric pair. Assume that $(\mathfrak{g}, \mathfrak{f})$ is not one of the pairs

(i) $(\mathfrak{S o}(2 n+k, \boldsymbol{C}), \mathfrak{S o}(n+k, \boldsymbol{C})+\mathfrak{S o}(n, \boldsymbol{C}))(n$ and $k$ a re odd $)$,

(ii) $(\mathfrak{S p}(2 n, \boldsymbol{C}), \mathfrak{F p}(n, \boldsymbol{C})+\mathfrak{F p}(n, \boldsymbol{C}))$.

Then $\left[K_{\theta}: K\right]$ coincides with the number of the irreducible components of $\mathcal{N}(V)$. On the other hand, in the cases (i) and (ii), $\left[K_{\theta}: K\right]=2$ and does not coincide with the number of the irreducible components of $\mathscr{N}(V)$.

For later use, we define

$\mathcal{N}(V)_{\text {reg }}=\left\{X \in \mathscr{N}(V) ; d P_{1}, \ldots, d P_{l}\right.$ are linearly independent at $\left.X\right\}$

$\mathscr{N}(V)_{p r}=\{X \in \mathscr{N}(V) ; X$ is principal $\}$

$\mathcal{N}(V)_{\text {sing }}=\mathscr{N}(V)-\mathscr{N}(V)_{\text {reg }}$.

It is clear that $\mathscr{N}(V)_{p r}$ is contained in $\mathscr{N}(V)_{\text {reg. }}$ But in general they do not coincide. We now give an example of such a pair that $\mathscr{N}(V)_{p r} \subsetneq \mathcal{N}(V)_{\text {reg }}$.

Example 1. 14. $(\mathfrak{I l}(n+1, \boldsymbol{C}), \mathfrak{g} \mathfrak{l}(n, \boldsymbol{C})) \quad(n \geqq 2)$.

Let $\mathfrak{g}=\mathscr{G l}(n+1, \boldsymbol{C})$ and let $\theta$ be an involution of $\mathfrak{g}$ defined by $\theta(X)=\left[\begin{array}{rr}I_{n} & 0 \\ 0 & -1\end{array}\right] X\left[\begin{array}{rr}I_{n} & 0 \\ 0 & -1\end{array}\right]$. Then $\mathfrak{f}=\{X \in \mathfrak{g} ; \theta(X)=X\}$ is isomorphic to the Lie algebra $\mathfrak{g l}(n, \boldsymbol{C})$. We identify $\boldsymbol{C}^{n} \times \boldsymbol{C}^{n}$ with $V=\{X \in \mathfrak{g}$;

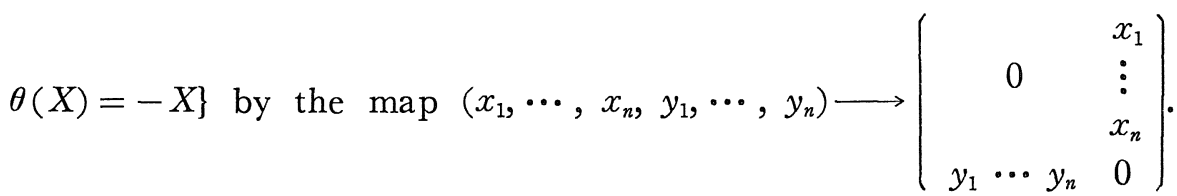
Under the identification, we find that

$$
\mathscr{N}(V)=\left\{(x, y) \in \boldsymbol{C}^{n} \times \boldsymbol{C}^{n} ; x_{1} y_{1}+\cdots+x_{n} y_{n}=0\right\} .
$$

By direct calculation, we also find that $\mathscr{N}(V)$ has four $K$-orbits $\mathcal{O}_{i}(i=1, \cdots, 4)$ defined by

$$
\begin{aligned}
& \mathcal{O}_{1}=\left\{(x, y) \in \boldsymbol{C}^{n} \times \boldsymbol{C}^{n} ; x_{1} y_{1}+\cdots+x_{n} y_{n}=0, x \neq 0, y \neq 0\right\} \\
& \mathcal{O}_{2}=\left\{(x, y) \in \boldsymbol{C}^{n} \times \boldsymbol{C}^{n} ; y \neq 0, x=0\right\} \\
& \mathcal{O}_{3}=\left\{(x, y) \in \boldsymbol{C}^{n} \times \boldsymbol{C}^{n} ; x \neq 0, y=0\right\} \\
& \mathcal{O}_{4}=\left\{(x, y) \in \boldsymbol{C}^{n} \times \boldsymbol{C}^{n} ; x=y=0\right\}
\end{aligned}
$$

and that $\mathcal{O}_{1}=\mathscr{N}(V)_{p r}, \mathcal{O}_{1} \cup \mathcal{O}_{2} \cup \mathcal{O}_{3}=\mathscr{N}(V)_{\text {reg. }}$. Hence $\mathscr{N}(V)_{p r} \subsetneq \mathscr{N}(V)_{\text {reg }}$ in this case. 
In general we have the following.

Theorem 4. Let $(\mathfrak{g}, \mathfrak{f})$ be a symmetric pair and let $\Sigma$ be its root system. Assume that $\mathrm{g}$ is simple.

(1) If $\Sigma$ is reduced, then $\mathscr{N}(V)_{p r}=\mathscr{N}(V)_{\text {reg }}$.

(2) If $\Sigma$ is not reduced, then $\mathcal{N}(V)_{p r} \cong \mathcal{N}(V)_{\text {reg. }}$. Moreover, in this case, if $X$ is in $\mathscr{N}(V)_{\text {reg }}-\mathscr{N}(V)_{\text {pr }}$, then $A d(G) X \cap V=$ $\mathscr{N}(V)_{\text {reg }}-\mathscr{N}(V)_{p r}$.

To prove Theorem 4, we need some preparations on certain properties of a general nilpotent element of $V$.

Let $X$ be a nilpotent element of $V$ and let $(H, X, Y)$ be a normal $S$-triple corresponding to $X$. For this $S$-triple, we set

(1. 1) $H^{\prime}=\sqrt{-1}(X-Y), X^{\prime}=\frac{1}{2}(X+Y+\sqrt{-1} H), Y^{\prime}=\frac{1}{2}(X+Y-\sqrt{-1} H)$.

Then it is clear that $\left(H^{\prime}, X^{\prime}, Y^{\prime}\right)$ is an $S$-triple and that $H^{\prime} \in V$. Now let $\mathfrak{a}$ be a Cartan subspace of $V$ which contains $H^{\prime}$ and let $\mathfrak{h}$ be a $\theta$-stable Cartan subalgebra of $g$ containing $\mathfrak{a}$. Furthermore let $\Phi$ be the root system of $(\mathfrak{g}, \mathfrak{h})$. We take a fundamental system $\Delta=\left\{\alpha_{1}\right.$, $\left.\cdots, \alpha_{L}\right\}$ such that $\alpha_{i}\left(H^{\prime}\right) \geqq 0(1 \leqq i \leqq L)$. Here $L$ is the rank of g. For any $\alpha \in \Phi$, we define $\alpha^{\theta}$ by $\alpha^{\theta}(A)=\alpha(\theta(A)) \quad(A \in \mathfrak{h})$. Then $\alpha^{\theta}$ is contained in $\Phi$. We set $\Phi_{-}=\left\{\alpha \in \Phi ; \alpha^{\theta}=\alpha\right\}$ and $\Delta \cap \Phi_{-}=$ $\left\{\alpha_{L-l_{-}{ }^{+}}, \cdots, \alpha_{L}\right\}$. Then there is a permutation $i \rightarrow i^{\prime}$ of order two of the set $\left\{1,2, \cdots, L-l_{-}\right\}$such that

(1. 2) $\quad \alpha_{i}^{\theta}=-\left(\alpha_{i^{\prime}}+\sum_{j=L_{-} l_{-}+1}^{L} n_{j}^{i} \alpha_{j}\right) \quad\left(n_{j}^{i} \in N, 1 \leqq i \leqq L-l_{-}\right)$

(cf. [W, Lemma 1.1.3.2]). Let $L-l_{-}=l_{1}+2 l_{2}, l_{1}+l_{2}=l$ (the rank of $(\mathfrak{g}, \mathfrak{f}))$. Then without loss of generality we may put

(1. 3) $\quad i^{\prime}= \begin{cases}i & \text { for } 1 \leqq i \leqq l_{1} \\ i+l_{2} & \text { for } l_{1}+1 \leqq i \leqq l_{1}+l_{2} \\ i-l_{2} & \text { for } l_{1}+l_{2}+1 \leqq i \leqq l_{1}+2 l_{2} .\end{cases}$

Putting $\lambda_{i}=\alpha_{i} \mid \mathfrak{a}(1 \leqq i \leqq l)$, we obtain a fundamental system $\left\{\lambda_{1}, \ldots, \lambda_{l}\right\}$ of the root system $\Sigma$.

Lemma 1.15. Under the above notation, we have 


$$
\begin{aligned}
& \alpha_{i}\left(H^{\prime}\right)=0 \quad\left(L-l_{-}+1 \leqq i \leqq L\right) \\
& \alpha_{i+l_{2}}\left(H^{\prime}\right)=\alpha_{i}\left(H^{\prime}\right) \quad\left(l_{1}+1 \leqq i \leqq l_{1}+l_{2}\right)
\end{aligned}
$$

Proof. First assume that $L-l_{-}+1 \leqq i \leqq L$. Then since $\theta\left(H^{\prime}\right)=$ $-H^{\prime}$, we have

$$
\alpha_{i}\left(H^{\prime}\right)=\alpha_{i}\left(-\theta\left(H^{\prime}\right)\right)=-\alpha_{i}^{\theta}\left(H^{\prime}\right)=-\alpha_{i}\left(H^{\prime}\right) .
$$

Hence $\alpha_{i}\left(H^{\prime}\right)=0$. Next assume that $l_{1}+1 \leqq i \leqq l_{1}+l_{2}$. Then it follows from (1.2), (1.3) and the argument above that

$$
\alpha_{i}\left(H^{\prime}\right)=-\alpha_{i}^{\theta}\left(H^{\prime}\right)=\alpha_{i^{\prime}}\left(H^{\prime}\right)=\alpha_{i+l_{2}}\left(H^{\prime}\right) . \quad \text { q. e. } d .
$$

Next we recall that the weighted Dynkin diagram $\Delta\left(X^{\prime}\right)$ of the nilpotent element $X^{\prime}$ is the Dynkin diagram of $\mathfrak{g}$ with the integer $\alpha_{i}\left(H^{\prime}\right)=0,1$ or 2 attached to the node corresponding to the root $\alpha_{i}(1 \leqq i \leqq L)$ (cf. [D], [B-C. p. 405]). Then we obtain a condition on the weighted Dynkin diagram of $X$.

Proposition 1. 16. Under the above notation, the weighted Dynkin diagram $\Delta(X)$ of $X$ has the following properties.

Let us consider the Satake diagram corresponding to a real form $\mathfrak{g}_{0}$ of $\mathfrak{g}$ such that $\mathfrak{f}_{0}=\mathfrak{H} \cap \mathfrak{g}_{0}$ is a maximal compact subalgebra of $\mathfrak{g}_{0}$. Then if the node corresponding to $\alpha_{i}$ is black, then the weight of $\alpha_{i}$ must be zero and if the nodes corresponding to $\alpha_{i}$ and $\alpha_{j}$ are connected by an arrow, then the weights of them must coincide.

Remark 1.17. The second condition in Proposition 1. 16 always holds for the weighted Dynkin diagram $\Delta(X)$ of any nilpotent element $X$ of $\mathrm{g}$ ([D]).

Proof. Since the condition (1.1) implies that $X$ and $X^{\prime}$ are $G$-conjugate, due to [B-C, Prop. 2.9], we have that $\Delta(X)=\Delta\left(X^{\prime}\right)$. This combined with Lemma 1.15 proves the proposition. q.e.d.

We now discuss on the converse of Proposition 1.16. In [S-S], the following proposition is stated. For completeness, we give here a proof of it. 
Proposition 1.18. Use the notation in Proposition 1.16. Assume that $\mathfrak{g}_{0}$ is a normal real form of $\mathfrak{g}$. Then for any nilpotent element $X$ of $\mathfrak{g}$, we have $\operatorname{Ad}(G) X \cap V \neq \emptyset$.

Proof. Since the assumption implies that $A d(G) X \cap g_{0} \neq \emptyset$, we may assume without loss of generality that $X$ is contained in $g_{0}$. Then Jacobson-Morozov lemma (cf. [Sp-St]) implies that there exist $H$, $Y \in \mathfrak{g}_{0}$ such that $(H, X, Y)$ is an $S$-triple. Then $H, X, Y$ form a subalgebra $\mathfrak{g}_{0}^{\prime}$ cf $\mathfrak{g}_{0}$ isomorphic to $\mathfrak{g l}(2, \boldsymbol{R})$. It is obvious that the automorphism $\theta^{\prime}$ of $\mathfrak{g}_{0}^{\prime}$ defined by

$$
\theta^{\prime}(H)=H, \theta^{\prime}(X)=-X, \theta^{\prime}(Y)=-Y
$$

is a Cartan involution of it. Then it follows from a theorem of Mostow (cf. [H-C]) that there exists a Cartan involution $\tilde{\theta}$ of $\mathfrak{g}_{0}$ such that the restriction of $\tilde{\theta}$ to $\mathfrak{g}_{0}^{\prime}$ coincides with $\theta^{\prime}$. Then from the conjugacy of maximal compact subalgebras, we may assume that $\tilde{\theta}=\theta$, namely that $\mathfrak{g}_{0}=\mathfrak{f}_{0}+V_{0}\left(V_{0}=V \cap \mathfrak{g}_{0}\right)$ is the Cartan decomposition corresponding to $\tilde{\theta}$. Thus we conclude that $X \in V_{0}$ and the proposition is proved.

q. e. d.

Remark 1.19. A generalization of Proposition 1.18 is obtained by Dr. L. Antonyon as stated below. The present author wishes his hearty thanks to Professor A. G. Elashvili who kindly informed him of the result in a letter.

Proposition 1. 18' (L. Antonyon). Let $X$ be a nilpotent element of $\mathrm{g}$ and let $(H, X, Y)$ be an $S$-triple corresponding to $X$. Then $A d(G) X \cap V \neq \varnothing$ if and only if $A d(G) H \cap V \neq \emptyset$.

To prove Theorem 4, we also use a transversal slice of the $K$ orbit of a nilpotent element of $V$ (for the definition of a transversal slice, see [Sl]). This plays a fundamental role in the study of the determination of the smoothly equivalent classes of the generic singularities of $\mathscr{N}(V)$ (cf. $\S \S 3$ and 4). We define a standard one and elementary properties of it.

Lemma 1.20. Let $X$ be a nilpotent element of $V$ and let $(H, X$, $Y)$ be a normal S-triple. We define $Z_{\mathrm{s}}(Y)=\{Z \in \mathfrak{g} ;[Z, Y]=0\}$. Then $V=[X, \mathfrak{l}]+V \cap Z_{\mathfrak{s}}(Y)$ is a direct sum decomposition. 
Proof. Easy.

Let $u_{1}, \ldots, u_{p}$ be a basis of $V \cap Z_{\mathfrak{s}}(Y)$ and let $v_{1}, \ldots, v_{q}$ be a basis of $\mathfrak{i} \cap Z_{\mathfrak{g}}(Y)$. Since $\operatorname{ad}(H)$ induces endomorphisms of $V \cap Z_{\mathfrak{g}}(Y)$ and $\mathfrak{i} \cap Z_{\mathfrak{g}}(Y)$, we may assume without loss of generality that $u_{1}, \ldots$, $u_{p}$ and $v_{1}, \ldots, v_{q}$ are eigenvectors of $\operatorname{ad}(H)$, in particular, we may set

$$
\begin{array}{ll}
{\left[H, u_{i}\right]=-n_{i} u_{i}} & (1 \leqq i \leqq p) \\
{\left[H, v_{j}\right]=-m_{j} v_{j}} & (1 \leqq j \leqq q) .
\end{array}
$$

Then it is known that $n_{1}, \ldots, n_{p}$ and $m_{1}, \ldots, m_{q}$ are non-negative integers (cf. $[\mathrm{H}-\mathrm{C}]$ ). Now we set $\mathscr{S}_{X}=X+V \cap Z_{\mathrm{g}}(Y)$.

Lemma 1.21. The subset $\mathscr{S}_{X}$ of $V$ is a transversal slice to the K-orbit of $X$ at $X$.

This lemma is proved by an argument similar to that in [Sl, p. 109].

Lemma 1. 22. Set $\delta=\chi \mid \mathscr{S}_{X}$. Then $\delta: \mathscr{S}_{X} \rightarrow \mathfrak{a} / W$ is a quasihomogeneous polynomial mapping of type $\left(d_{1}, \ldots, d_{l} ; w_{1}, \ldots, w_{p}\right)$. Here $d_{1}, \ldots, d_{l}$ are the degrees of $P_{1}, \ldots, P_{l}$ and $w_{i}=\frac{n_{i}}{2}+1(1 \leqq i \leqq p)$.

For the definition of a quasihomogeneous polynomial mapping, see [Sl, p. 109]. The proof of the lemma is similar to that of [Sl, 7. 4. Prop. 1]. Hence we omit it.

For laler use, we define

$$
f_{j}\left(y_{1}, \ldots, y_{p}\right)=P_{j}\left(X+\sum_{i=1}^{p} y_{i} u_{i}\right) \quad(1 \leqq j \leqq l) .
$$

Then from the definition, $f_{j}(y)$ is a quasihomogeneous polynomial of type $\left(d_{j} ; w_{1}, \ldots, w_{p}\right)$.

Lemma 1.23. Let $X$ be a nilpotent element of $V$. Use the notation in Lemma 1.22. Assume that $w_{1}, \ldots, w_{p}<d_{l}$. Then the 1-form $d P_{l}$ vanishes at $X$.

Proof. Since $d_{l}$ is maximal among $d_{1}, \ldots, d_{l}$, it follows from the quasihomogeneity that $f_{l}(y)$ has no linear terms. Hence $\left.\left(d f_{l}\right)\right|_{y=0}=0$. This and the definition of the transversal slice $\mathscr{S}_{X}$ show that $\left(d P_{l}\right)_{X}$ $=0$.

q.e.d. 
Proposition 1.24. Use the notation above. Let $\tilde{\alpha}=\sum_{i=1}^{L} r_{i} \alpha_{i}$ be the maximal root of $\Phi$. Let $X$ be nilpotent of $V$ and let $(H, X, Y)$ be a corresponding normal S-triple. If $\frac{1}{2} \tilde{\alpha}\left(H^{\prime}\right)+1<d_{l}$, then $X$ is contained in $\mathscr{N}(V)_{\text {sing }}$ (for the definition of $H^{\prime}$, see (1.1)).

Proof. It is clear that if $m$ is an eigenvalue of $a d\left(H^{\prime}\right)$, then $|m| \leqq \tilde{\alpha}\left(H^{\prime}\right)$. On the other hand, since $H$ and $H^{\prime}$ are $G$-conjugate, the eigenvalues of $a d(H)$ and $a d\left(H^{\prime}\right)$ coincide. Noting that $n_{1}, \ldots$, $n_{p}$ are eigenvalues of $a d(H)$, we have that $n_{i} \leqq \tilde{\alpha}(H)$ and therefore that $w_{i}=\frac{n_{i}}{2}+1 \leqq \frac{1}{2} \tilde{\alpha}(H)+1$. Thus Lemma 1.23 proves the proposition.

q.e.d.

Proof of Theorem 4. (1) We consider a nilpotent element $X$ of $V$. Let $(H, X, Y)$ be a corresponding normal $S$-triple and let $\left(H^{\prime}, X^{\prime}, Y^{\prime}\right)$ be defined as in (1.1).

First we assume that $X$ is principal nilpotent. Then it follows from Proposition 1.16 and [K-R, Prop. 13] that the weighted Dynkin diagram $\Delta(X)$ of $X$ is determined in the following way: Consider the Satake diagram corresponding to the pair $(\mathfrak{g}, \mathfrak{f})$. If a node of the Satake diagram is white (resp. black), then the weight for it is 2 (resp. 0). Noting this, we conclude by the classification that $\frac{1}{2} \widetilde{\alpha}\left(H^{\prime}\right)$ $+1=d_{l}$. Here we used the concrete expression of the Satake diagrams (cf. [W, p. 30]) and those of the maximal roots (cf. [LIE]). For readers' convenience, we give here some examples so that the equality $\frac{1}{2} \tilde{\alpha}\left(H^{\prime}\right)+1=d_{l}$ actually holds.

(i) $\quad(\mathfrak{l}(n, \boldsymbol{C}), \mathfrak{S O}(n, \boldsymbol{C}))$

The Satake diagram of the pair is $\begin{array}{cccc}\alpha_{1} & \alpha_{2} & \ldots & \alpha_{n-1} \\ 0 & & 0 . & 0\end{array}$ case, $\Delta(X)$ is $22 \cdots 2$. Furthermore, the maximal root is $\tilde{\alpha}=\alpha_{1}+\cdots$ $+\alpha_{n-1}$. Hence $\tilde{\alpha}\left(H^{\prime}\right)=2(n-1)$ and therefore $\frac{1}{2} \tilde{\alpha}\left(H^{\prime}\right)+1=n$ which is nothing but the maximum of the degrees of the basic $K$-invariants.

(ii) $\quad\left(\mathfrak{e}_{7}^{c}, \mathfrak{e}_{6}^{c}+\boldsymbol{C}\right)$

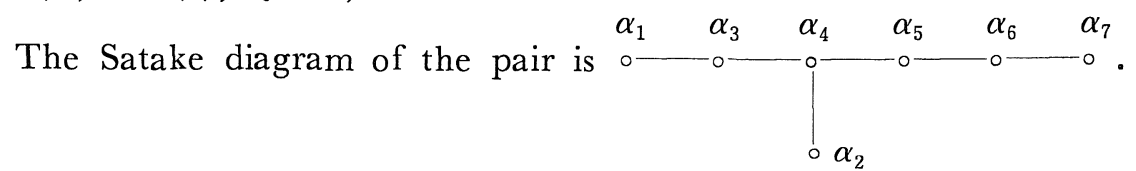


In this case, the maximal root is $\tilde{\alpha}=2 \alpha_{1}+2 \alpha_{2}+3 \alpha_{3}+4 \alpha_{4}+3 \alpha_{5}+2 \alpha_{6}+$ $\alpha_{7}$. Hence $\frac{1}{2} \tilde{\alpha}\left(H^{\prime}\right)+1=6$. On the other hand, the degrees of the basic $K$-invariants are $2,4,6$. These imply the equality in this case.

(iii) $\quad\left(\mathfrak{e}_{8}^{c}, \mathfrak{e}_{7}^{c}+\mathfrak{G l}(2, \boldsymbol{C})\right)$

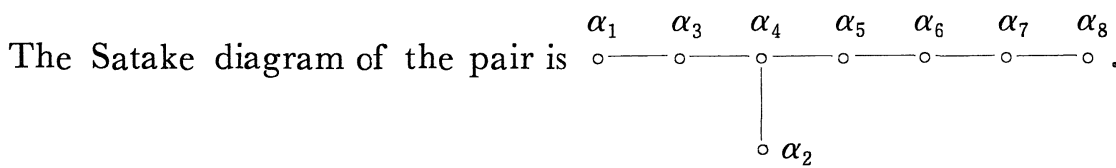

In this case the maximal root is $\tilde{\alpha}=2 \alpha_{1}+3 \alpha_{2}+4 \alpha_{3}+6 \alpha_{4}+5 \alpha_{5}+4 \alpha_{6}+$ $3 \alpha_{7}+2 \alpha_{8}$. Hence $\frac{1}{2} \tilde{\alpha}\left(H^{\prime}\right)+1=12$. On the other hand, since the root system of the pair is of type $F_{4}$, the degrees of the basic $K$-invariants are 2, 6, 8, 12 .

Next we assume that $X$ is not principal nilpotent. Then from Proposition 1.16 and the above discussion, it follows that $\frac{1}{2} \tilde{\alpha}\left(H^{\prime}\right)+$ $1<d_{l}$. Then due to Proposition 1.24, we conclude that $X$ is in $\mathcal{N}(V)_{\text {sing. }}$

Hence we have proved (1).

(2) We first recall all the symmetric pairs whose root systems are not reduced. They are contained in Table III.

Table III

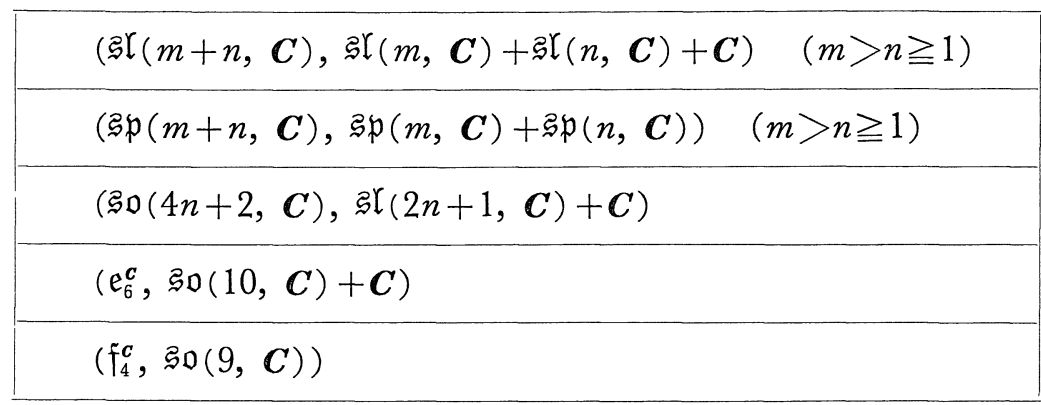

We will prove the claim in (2) by using the classification. If $\mathfrak{g}$ is of classical type, the proof of (2) is not difficult. Hence we may assume that $\mathrm{g}$ is of exceptional type.

First we show (2) in the case when $(\mathfrak{g}, \mathfrak{k})=\left(\mathfrak{e}_{6}^{\boldsymbol{c}}, \mathfrak{g} \mathfrak{0}(10, \boldsymbol{C})\right)$. Since every nilpotent element $\mathrm{cf} \mathfrak{g}$ is characterized by its weighted Dynkin diagram, we now enumerate the weighted Dynkin diagrams 
which satisfies the conditions in Proposition 1.16. They are as follows (cf. [D, B-C, E]) :

$\begin{array}{cccccccc}20002 & 10001 & 20002 & 10001 & 00000 & 10001 & 00000 & 00000 \\ 2 & 2 & 0 & 1 & 2 & 1 & 1 & 0\end{array}$

It follows from Lemma 1.23 that if $X$ is a nilpotent element and $\Delta(X)$ is one of the above weighted Dynkin diagrams except 20002,

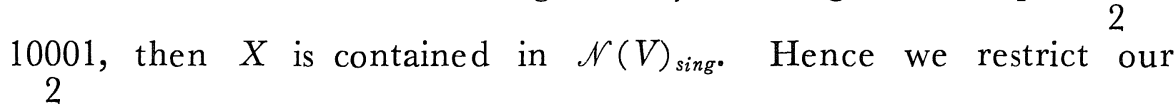

attention to the nilpotent elements of $\mathfrak{g}$ whose weighted Dynkin diagram is one of 20002, 10001. It easily follows from [K-R, Prop. 13] 22

that there exists a nilpotent element $X$ of $V$ such that $\Delta(X)=20002$. This is principal nilpotent. Next we show that there exists a nilpotent element $X$ of $V$ whose weighted Dynkin diagram is 10001. For this purpose, we take a Cartan subalgebra $\mathfrak{h}$ of $\mathfrak{g}$. Let $\Phi^{2}$ be the root system of $(\mathfrak{g}, \mathfrak{h})$ and let $\left\{X_{\alpha} ; \alpha \in \Phi\right\}$ be a Weyl basis of $\mathfrak{g} \bmod \mathfrak{h}$ (cf. [W, 1.1.1]). Let $\Delta=\left\{\alpha_{1}, \alpha_{2}, \alpha_{3}, \alpha_{4}, \alpha_{5}, \alpha_{6}\right\}$ be a fundamental system

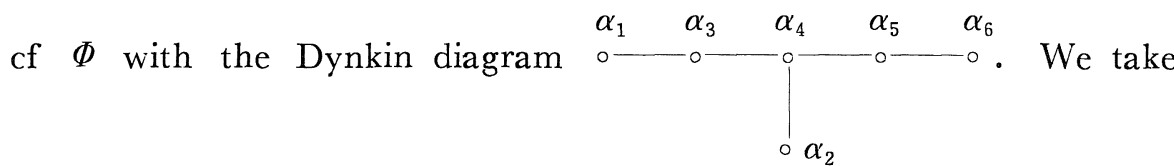
out a signature $\varepsilon: \Phi \rightarrow\{1,-1\}$ of roots defined by $\varepsilon\left(\alpha_{1}\right)=\varepsilon\left(\alpha_{2}\right)=$ $-1, \varepsilon\left(\alpha_{i}\right)=1(i=3,4,5,6)$ (cf. [O-S]). Then we define an involution $\theta$ of $g$ by

$$
\begin{array}{ll}
\theta\left(X_{\alpha}\right)=\varepsilon(\alpha) X_{\alpha} & (\forall \alpha \in \Phi) \\
\theta(H)=H & (\forall H \in \mathfrak{G}) .
\end{array}
$$

The subalgebra $\mathfrak{f}=\{X \in \mathfrak{g} ; \theta(X)=X\}$ is spanned by $\mathfrak{h}$ and $\left\{X_{\alpha}\right.$; $\alpha \in \Phi, \varepsilon(\alpha)=1\}$. It follows from the definition that $\#\{\alpha \in \Phi ; \varepsilon(\alpha)=1\}$ $=40$ and therefore that $\operatorname{dim} \mathfrak{f}=46$. Since $(\mathfrak{g}, \mathfrak{f})$ is a complex symmetric pair, this implies that is isomorphic to $\mathfrak{g} \mathfrak{O}(10, \boldsymbol{C})+\boldsymbol{C}$. Let $H_{0}$ be the element of $\mathfrak{h}$ such that $\alpha_{1}\left(H_{0}\right)=\alpha_{6}\left(H_{0}\right)=1, \alpha_{2}\left(H_{0}\right)=2, \alpha_{3}\left(H_{0}\right)=$ $\alpha_{4}\left(H_{0}\right)=\alpha_{5}\left(H_{0}\right)=0$. If $\mathfrak{g}(2)=\left\{X \in \mathfrak{g} ;\left[H_{0}, X\right]=2 X\right\}$, then from the definition $\mathfrak{g}(2)$ is contained in $V$. On the other hand, there exists a nilpotent element $X$ of $\mathfrak{g}(2)$ whose weighted Dynkin diagram is 10001. In fact such nilpotent elements are open dense in $\mathfrak{g}(2)$. We 2 have thus proved (2) in this case. 
Next we consider the case $(\mathfrak{g}, \mathfrak{f})=\left(\mathfrak{f}_{4}^{c}, \mathfrak{g} \mathfrak{p}(9, \boldsymbol{C})\right)$. Let $\mathfrak{h}, \Phi$, $\left\{X_{\alpha} ; \alpha \in \Phi\right\}$ be defined similarly as in the previous case. Let $\Delta=$ $\left\{\alpha_{1}, \alpha_{2}, \alpha_{3}, \alpha_{4}\right\}$ be a fundamental system of $\Phi$ with the diagram $\alpha_{1} \quad \alpha_{2} \quad \alpha_{3} \quad \alpha_{4}$. We enumerate the weighted Dynkin diagrams which satisfies the condition in Proposition 1.16. They are as follows (cf. [D]): $00 \Rightarrow 02,00 \Rightarrow 01,00 \Rightarrow 00$. We take out a signature $\varepsilon: \Phi \rightarrow$ $\{1,-1\}$ defined $\varepsilon\left(\alpha_{1}\right)=\varepsilon\left(\alpha_{2}\right)=\varepsilon\left(\alpha_{3}\right)=1, \quad \varepsilon\left(\alpha_{4}\right)=-1$ and as in the previous case we define an involution $\theta$ of $\mathfrak{g}$ using $\varepsilon$. Then it is easy to see that the corresponding symmetric pair $(\mathfrak{g}, \mathfrak{f})$ is isomorphic to $\left(\mathfrak{f}_{4}^{c}, \mathfrak{S} \mathfrak{D}(9, \boldsymbol{C})\right)$. We only prove the existence of a nilpotent element of $V$ whose weighted Dynkin diagram is $00 \Rightarrow 01$. The rest of the proof is similar to the above case. Let $X=X_{\alpha_{4}}$. Then from the definition, $X$ is nilpotent and is contained in $V$. By an elementary computation, we conclude that $\Delta(X)=00 \Rightarrow 01$ and that $X \in \mathscr{N}(V)_{\text {reg. }}$. Hence the claim (2) is completely proved. q. e. d.

\section{§2. A Resolution of the Nilpotent Subvariety}

In this section, we construct a manifold $\tilde{\mathscr{N}}(V)$ which is an analogue to $\mathscr{N}(V)$ of the Springer's resolution of the nilpotent subvariety of a simple Lie algebra (cf. [Sp, St]).

Let $X_{0}$ be a principal nilpotent element of $V$ and let $\left(H_{0}, X_{0}, Y_{0}\right)$ be a normal $S$-triple corresponding to $X_{0}$. We define

$$
\begin{aligned}
& \mathfrak{g}(j)=\left\{A \in \mathfrak{g} ;\left[H_{0}, A\right]=j A\right\}, \\
& \mathfrak{i}=\bigoplus_{j \geq 0} \mathfrak{g}(j), \quad \mathfrak{n}=\bigoplus_{j>0} \mathfrak{g}(j), \\
& \mathfrak{l}=\mathfrak{E} \cap \mathfrak{\mathfrak { l }}, \quad \mathfrak{n}=V \cap \tilde{\mathfrak{n}} .
\end{aligned}
$$

We note here that $\mathfrak{f}$ is a parabolic subalgebra of $\mathfrak{g}$, that $\mathfrak{\mathfrak { n }}$ is the nilpotent radical and that $[\tilde{f}, \tilde{\mathfrak{n}}] \subseteq \tilde{\mathfrak{n}}$. Let $\widetilde{L}$ be the parabolic subgroup cf $G$ corresponding to $\left\{\right.$ and let $L_{\theta}=\tilde{L} \cap K_{\theta}$. We discuss on $L_{\theta}$ later.

Lemma 2. 1. $\operatorname{dim} \operatorname{Ad}(G) X_{0}=2 \operatorname{dim} \tilde{\mathfrak{n}}$.

Proof. We define $H_{0}^{\prime}, X_{0}^{\prime}, Y_{0}^{\prime}$ from $H_{0}, X_{0}, Y_{0}$ as in (1.1) and also define $\mathfrak{g}(j)^{\prime}, \mathfrak{f}^{\prime}, \tilde{\mathfrak{n}}^{\prime}$ as in (2.1) by using $H_{0}^{\prime}$ instead of $H_{0}$. Since $H_{0}$ and $H_{0}^{\prime}$ are $G$-conjugate, to prove Lemma 2.1, it suffices to show that $\operatorname{dim} \operatorname{Ad}(G) X_{0}^{\prime}=2 \operatorname{dim} \tilde{n}^{\prime}$. But it follows from [K-R, Prop. 8] that 


$$
\begin{aligned}
\operatorname{dim} \operatorname{Ad}(G) X_{0}^{\prime} & =\operatorname{dim} \mathfrak{g}-\operatorname{dim} Z_{\mathfrak{g}}\left(X_{0}^{\prime}\right) \\
& =\operatorname{dim} \mathfrak{g}-(\operatorname{dim} \mathfrak{a}+\operatorname{dim} \mathfrak{m}) \\
& =2 \operatorname{dim} \tilde{\mathfrak{n}}^{\prime} .
\end{aligned}
$$

(Here $\mathfrak{a}($ resp. $\mathfrak{m})$ is the centralizer of $H_{0}^{\prime}$ in $V$ (resp. $\left.\mathfrak{f}\right)$ ). q. e. d.

Remark 2.2. Lemma 2.1 shows that the parabolic subgroup $\widetilde{L}$ of $G$ is a polarization of $X_{0}$ (cf. [Hes 2]).

Every element $p$ of $L_{\theta}$ induces an automorphism of $K_{\theta} \times \mathfrak{\pi}$ in the following way: $(k, X) \rightarrow\left(k p, A d\left(p^{-1}\right) X\right)$. We denote by $\tilde{N}(V)$ the quotient of $K_{\theta} \times \mathfrak{n}$ by the action of $L_{\theta}$ and put $k * X=(k, X) L_{\theta}$ for any $(k, X) \in K_{\theta} \times \mathfrak{n}$. Let $\pi$ be the canonical mapping of $\tilde{\mathscr{N}}(V)$ to $\mathscr{N}(V)$. By the construction, connected components of $\tilde{\mathscr{N}}(V)$ correspond to irreducible components of $\mathscr{N}(V)$. Hence in general $\tilde{N}(V)$ is not connected (cf. Theorem 1).

Lemma 2. 3. The centralizer $Z_{G}\left(X_{0}\right)=\left\{g \in G ; A d(g) X_{0}=X_{0}\right\}$ of $X_{0}$ is contained in $\widetilde{L}$.

Proof. We first show that $\widetilde{L}$ is independent of the choice of $H_{0}$ and $Y_{0}$. Let $\left(H_{0}^{\prime}, X_{0}, Y_{0}^{\prime}\right)$ be another $S$-triple. Then it follows from [Ko 1, Th. 3.6] that there exists an element $g$ of $Z_{G}\left(X_{0}\right)^{0}$ such that $H_{0}^{\prime}=A d(g) H_{0}$, where $Z_{G}\left(X_{0}\right)^{0}$ is the identity component of $Z_{G}\left(X_{0}\right)$. Since the Lie algebra of $Z_{G}\left(X_{0}\right)$ is clearly contained in $f$, it follows that $Z_{G}\left(X_{0}\right)^{0} \subset \widetilde{L}$. This implies that $g$ is in $\tilde{L}$. If $f^{\prime}$ is the parabolic subalgebra of $\mathrm{g}$ defined from $\left(H_{0}^{\prime}, X_{0}, Y_{0}^{\prime}\right)$ by a method similar to the constrution of $\mathfrak{i}$, then clearly $\mathfrak{i}^{\prime}=A d(g) \mathfrak{i}=\mathfrak{i}$. This means that $\mathfrak{f}$ does not depend on the choice of $H_{0}, Y_{0}$.

We are going to prove the proposition. Let $g$ be an element of $Z_{G}\left(X_{0}\right)$. Then we can define a parabolic subalgebra $A d(g)\lceil$ from $\left(A d(g) H_{0}, A d(g) X_{0}, A d(g) Y_{0}\right)$ as we did in (2.1). Since $A d(g) X_{0}=$ $X_{0}$, it follows from the above argument that $A d(g)\lceil=\{$. This implies that $g \in N_{G}(\tilde{i})=N_{G}(\tilde{L})=\widetilde{L}$. Hence the result.

q.e.d.

Remark 2.4. Proposition 2.3 holds for a general nilpotent element of $\mathrm{g}$. The proof employed here is the one given in [Sp-St]. Mr. Tanisaki kindly informed the author of the proof. 
Lemma 2.5. If $X$ is contained in $\mathcal{N}(V)_{p r}$, then $\pi^{-1}(X)$ consists of one point.

Proof. Owing to [K-R, Th. 6], we may assume that $X=X_{0}$. We consider the homogeneous vector bundle $G \times^{\mathcal{L}} \tilde{\mathfrak{n}}$ over $G / \tilde{L}$ defined similarly as $\tilde{\mathscr{N}}(V)$. Since $a d\left(X_{0}\right):\{\rightarrow \tilde{\mathfrak{n}}$ is a surjective linear transformation, Lemma 2.3 implies that $\tilde{\pi}: G \times^{\mathcal{L}} \tilde{\mathfrak{n}} \rightarrow \overline{A d(G) X_{0}}$ is birational and $\tilde{\pi}^{-1}\left(X_{0}\right)=\left\{e * X_{0}\right\}$. Since $e * X_{0} \in \pi^{-1}\left(X_{0}\right)$ and since $\pi^{-1}\left(X_{0}\right)$ is identified with a subset of $\tilde{\pi}^{-1}\left(X_{0}\right)$, we conclude that $\pi^{-1}\left(X_{0}\right)=\left\{e * X_{0}\right\}$. q.e.d.

The following theorem shows that $\tilde{\mathscr{N}}(V)$ is an analogue of the Springer's resolution of the nilpotent variety of a simple Lie algebra (cf. [Sp, St]).

Theorem 5. The mapping $\pi: \tilde{\mathscr{N}}(V) \rightarrow \mathscr{N}(V)$ has the following properties:

(a) $\tilde{\mathscr{N}}(V)$ is smooth,

(b) $\pi$ is proper and surjective,

(c) $\pi$ induces an isomorphism $\pi^{-1}\left(\mathcal{N}(V)_{p r}\right) \rightarrow \mathscr{N}(V)_{p r}$.

Proof. Lamma 2.5 implies that $\pi^{-1}\left(\mathcal{N}(V)_{p r}\right) \rightarrow \mathcal{N}(V)_{p r}$ is one to one. Noting this, we can prove the theorem by an argument similar to that of [Sl, 3.9, Th. 1]. Hence we omit it.

q. e. d.

Corollary. For any nilpotent element $X$ of $V$, we have $A d\left(K_{\theta}\right) X \cap \mathfrak{n} \neq \phi$.

Proof. Since $\pi$ is surjective, there exists an element $\left(k, X^{\prime}\right)$ of $K \times \mathfrak{n}$ such that $X=\pi\left(k * X^{\prime}\right)=A d(k) X^{\prime}$. This implies the claim.

q. e. d.

Remark 2.6. A. Grothendieck constructed a simultaneous resolution of a simple Lie algebra which contains the Springer's resolution (that is, the restriction of it to the nilpotent variety coincides with the Springer's resolution) and plays a fundamental role in the construction of the simultaneous resolution of the versal deformations of the rational double points (cf. [Sl]). Hence it is natural to ask 
whether there exists a simultaneous resolution of $V$ analogous to the one due to Grothendieck or not. The present author cannot give an answer to the problem at present.

In order to obtain a property of the transversal slices to $K$-orbits of $\mathcal{N}(V)$, we state a theorem on a mapping between varieties on each of which an algebraic group acts.

Theorem 7 ( $P$. Slodowy). Assume that $G$ is an algebraic group and that $\pi: Y \rightarrow X$ is a G-equivariant mapping of G-varieties. Let $S \subset X$ be a transversal slice to some $G$-orbit $G \cdot x$ in $X$. Then we have

(a) If $Y$ is smooth, then $\pi^{-1}(S)$ is smooth.

(b) If $\pi$ is birational, then $\pi^{-1}(S) \rightarrow S$ is birational.

(c) If $\pi$ is a resolution of the singularities, then so is $\pi^{-1}(S) \rightarrow S$.

Theorem 7 follows from the arguments in [S1, pp. 60-66].

Remark 2.7. The present author wishes his hearty thanks to Prof. P. Slodowy who kindly informed him of the theorem above as well as its proof in a letter.

We apply Theorem 7 to our situation. Let $X_{0}$ be the principal nilpotent element as before and let $\mathscr{N}(V)_{1}=\overline{A d(K) X_{0}}$, the Zariski closure of the $K$-orbit of $X_{0}$ which is obviously irreducible. Let, further, $\tilde{\mathscr{N}}(V)_{1}$ be the connected component of $\tilde{\mathscr{N}}(V)$ containing $e * X_{0}$. Then $\pi \mid \tilde{N}(V)_{1}$ is a resolution of $\mathscr{N}(V)_{1}$. We now take out any nilpotent element of $\mathscr{N}(V)_{1}$. Then due to Corollary to Theorem 5, we may assume that $X$ is contained in $\mathfrak{n}$. Let $\mathscr{S}_{X}$ be a transversal slice to the $K$-orbit of $X$ at $X$. Then from the definition, it follows that $\mathscr{S}^{\prime}=\mathscr{S}_{X} \cap \mathscr{N}(V)_{1}$ is a transversal slice to the $K$-orbit of $X$ at $X$ in $\mathscr{N}(V)_{1}$.

Proposition 2. 8. Under the notation above, put $\pi_{1}=\pi \mid \tilde{N}(V)_{1}$. Then $\pi_{1}^{-1}(\mathscr{S})$ is smooth and $\pi_{1}^{-1}\left(\mathscr{S}^{\prime}\right) \rightarrow \mathscr{S}^{\prime}$ is a resolution of the singularities of $\mathscr{S}^{\prime}$.

Proposition 2.8 is a direct consequence of Theorem 7 . 
Remark 2.9. The manifold $\tilde{\mathscr{N}}(V)_{1}$ is constructed in a way similar to the construction of $\tilde{\mathscr{N}}(V)$ by taking $L=L_{\theta} \cap K$ instead of $L_{\theta}$. If $K_{s}$ is the commutator subgroup of $K$, then $L \cap K_{s}$ is obviously a parabolic subgroup of $K_{s}$.

We give here examples which illustrate the resolution of the nilpotent variety.

Example 2. 10. $(\mathfrak{S D}(n+1, \boldsymbol{C}), \mathfrak{g} \mathfrak{D}(n, \boldsymbol{C})) \quad(n \geqq 2)$.

In this case, $V$ is identified with $\boldsymbol{C}^{n}$ and the nilpotent subvariety $\mathscr{N}(V)$ with the set

$$
S=\left\{x \in \boldsymbol{C}^{n} ; x_{1}^{2}+\cdots+x_{n}^{2}=0\right\} .
$$

Then the resolution $\tilde{\mathscr{N}}(V)$ is identified with

$$
\begin{gathered}
\tilde{S}=\left\{(x, \xi) \in \boldsymbol{C}^{n} \times \boldsymbol{P}^{n-1} ; x_{1}^{2}+\cdots+x_{n}^{2}=0,\right. \\
\left.\xi_{1}^{2}+\cdots+\xi_{n}^{2}=0, \quad x \in \xi\right\} .
\end{gathered}
$$

Define $S_{1}=\{x \in S ; x \neq 0\}$ and $S_{2}=\{0\}$. Then the $K$-orbits of $S$ are $S_{1}$ and $S_{2}$. If $x$ is contained in $S_{1}$, then $\pi^{-1}(x)$ is clearly a single point. On the other hand, if $x=0$, then $\pi^{-1}(x)=\boldsymbol{P}^{n-1}$.

\section{Example 1. 14 (continued).}

In this case, $V$ is identified with $\boldsymbol{C}^{2 n}$ and $\mathscr{N}(V)$ is with the set

$$
S=\left\{(x, y) \in \boldsymbol{C}^{n} \times \boldsymbol{C}^{n} ; x_{1} y_{1}+\cdots+x_{n} y_{n}=0\right\} .
$$

The resolution $\tilde{\mathscr{N}}(V)$ is identified with

$$
\begin{gathered}
\tilde{S}=\left\{((x, y),(\xi, \eta)) \in S \times \boldsymbol{P}^{n-1} \times \boldsymbol{P}^{n-1} ; \xi_{1} \eta_{1}+\cdots+\xi_{n} \eta_{n}=0,\right. \\
x \in \xi, y \in \eta\} .
\end{gathered}
$$

We may regard $\mathcal{O}_{i}(i=1,2,3,4)$ as subsets of $S$. Then $S_{i}(i=1$, ..., 4) are the $K$-orbits of $S$. In particular, $S_{1}$ is the totality of the principal nilpotent elements and $S^{\prime}=S_{1} \cup S_{2} \cup S_{3}$ is non-singular and identified with $\mathscr{N}(V)_{\text {reg. }}$. If $(x, y)$ is in $S_{1}$, then $\pi^{-1}((x, y))$ is a single point. On the other hand, for any $x \in \boldsymbol{C}^{n}(x \neq 0)$, we have $\pi^{-1}((x, 0))=\boldsymbol{P}^{n-2}$. Moreover $\pi^{-1}(0,0)=\boldsymbol{P}^{n-1} \times \boldsymbol{P}^{n-1}$.

In Example 1.14 (continued), we find that the fibre of every nilpotent element of $\mathscr{N}(V)_{\text {reg }}-\mathscr{N}(V)_{p r}$ is isomorphic to a projective space. In general, we observe the following. 
Proposition 2. 11. Let $(\mathfrak{g}, \mathfrak{f})$ be a symmetric pair. Assume that $\mathfrak{g}$ is of classical type and that the root system $\Sigma$ of $(\mathfrak{g}, \mathfrak{l})$ is not reduced. Let $\alpha$ be a root of $\Sigma$ such that $2 \alpha$ is also a root of $\Sigma$. We denote by $2 m$ the multiplicity of $\alpha$. (Note that $m$ is an integer and does not depend on the choice of $\alpha$.)

Let $X$ be any element of $\mathscr{N}(V)_{\text {reg }}-\mathcal{N}(V)_{\text {pro }}$ Then we have

$$
\operatorname{dim}(\operatorname{Ad}(K) X)=m, \pi^{-1}(X)=\mathbb{P}^{m-1} .
$$

Proof. We prove the proposition by using the classification in Table II. As for the dimension of the $K$-orbit of $X$, we can prove the equality by examining $\operatorname{dim}\left(Z_{\mathrm{g}}(X) \cap V\right)$ (cf. $\left.\S 3, \S 4\right)$. We determine the fibre of $X$. Since $(\mathfrak{g}, \mathfrak{f})$ is of classical type, the claim is easily checked by direct calculation. q.e. d.

Remark 2.12. We conjecture that Proposition 2.11 holds even if we do not assume that $g$ is of classical type.

We give here an identification of $\tilde{\mathscr{N}}(V)$ with a subset of $\left(K_{\theta} / L_{\theta}\right) \times$ $\mathscr{N}(V)$. The identification will be used in the following section. We define a mapping $\iota$ of $\tilde{\mathscr{N}}(V)$ into $\left(K_{\theta} / L_{\theta}\right) \times \mathscr{N}(V)$ by $\iota(k * X)=\left(k L_{\theta}\right.$, $\left.A d\left(k^{-1}\right) X\right)$. Then the following are direct consequences of the definitions of $\tilde{\mathscr{N}}(V)$ and $\iota$ :

(i) $c$ is an imbedding,

(ii) $\iota(\tilde{\mathscr{N}}(V))=\left\{\left(k L_{\theta}, X\right) \in\left(K_{\theta} / L_{\theta}\right) \times \mathscr{N}(V) ; A d\left(k^{-1}\right) X \in \mathfrak{n}\right\}$,

(iii) For any $X \in \mathcal{N}(V)$, the fibre $\pi^{-1}(X)$ is identified with the set $u(X)=\left\{k L_{\theta} \in K_{\theta} / L_{\theta} ; A d\left(k^{-1}\right) X \in \mathfrak{n}\right\}$.

In the rest of this section, we restrict our attention to the subalgebra $\mathfrak{l}$ of $\mathfrak{i}$. Noting that $\mathfrak{E}$ is not semisimple in general, we put $\mathfrak{f}=\mathfrak{l}_{s}+t$, where $\mathfrak{l}_{s}$ is the semisimple part of $\mathfrak{i}$ and $t$ is the center of $\mathfrak{t}$. Then it follows from the definition that $\mathfrak{l}$ contains $\mathfrak{t}$ and that $\mathfrak{l}_{s}=\mathfrak{l} \cap \mathfrak{l}_{s}$ is a parabolic subalgebra of $\mathfrak{f}_{s}$. Since there are many non-conjugate parabolic subalgebras of $\mathfrak{f}_{s}$, it seems to be of some importance to determine the type of $\mathfrak{l}_{s}$. From now on we attempt to accomplish this. First we give the following proposition.

Proposition 2.13. If $V$ contains a regular semisimple element of $\mathfrak{g}$, that is, if the Satake diagram of $(\mathfrak{g}, \mathfrak{f})$ contains only white 
nodes, then $\mathfrak{l}_{s}$ is a Borel subalgebra of $\mathfrak{f}_{s}$.

Proof. It follows from the assumption and [K-R, Prop. 13] that $\mathscr{N}(V)$ contains a regular nilpotent element of $\mathfrak{g}$. Accordingly, if $X$ is a regular nilpotent element of $\mathfrak{g}$ contained in $V$ and $(H, X, Y)$ is a corresponding normal $S$-triple, it follows that $H$ is a regular semisimple element of $\mathfrak{g}$. Then we find from (2.1) that $f$ is a Borel subalgebra of $\mathfrak{g}$. This implies that $\mathfrak{l}=\mathfrak{i} \cap$ is a direct sum of $\mathfrak{t}$ and a Borel subalgebra of $\mathfrak{f}_{\text {s. }}$ Hence the result.

q. e. d.

From now on we are going to determine $\mathfrak{l}_{s}$ when $\mathfrak{g}$ is simple of classical type. Let $(H, X, Y)$ be a normal $S$-triple, where $X$ is principal nilpotent of $\mathscr{N}(V)$ and $\mathfrak{l}$ is determined from $H$ as in (2.1). Let $\mathfrak{b}$ be a Cartan subalgebra of $\mathfrak{f}_{s}$ and let $\Sigma_{\mathfrak{t}}$ be the root system concerning $(\mathfrak{f}, \mathfrak{b})$. We take a fundamental system of roots $\Delta_{\mathfrak{t}}$ of $\Sigma_{\mathfrak{t}}$ such that $\alpha(H) \geqq 0$ for any $\alpha \in \Delta_{\mathfrak{t}}$. Put $\Delta_{\mathfrak{t}}^{-}=\left\{\alpha \in \Delta_{\mathfrak{t}} ; \alpha(H)=0\right\}$. Then $\mathfrak{l}_{s}$ is determined from $\Delta_{t}^{-}$as follows. Let $\left\{X_{\alpha} ; \alpha \in \Sigma_{t}\right\}$ be a Weyl basis of $\mathfrak{f}_{s} \bmod \mathfrak{b}$ and put $\Sigma_{\mathfrak{t}}^{\prime}=\Sigma_{\mathfrak{t}} \cap\left(\sum_{\alpha \in \Delta_{\mathfrak{t}}^{-}} \boldsymbol{R} \alpha\right)$. Then $\mathfrak{I}_{s}=\mathfrak{b}+\sum_{\alpha \in \Sigma_{\mathfrak{t}}^{\prime}} \boldsymbol{C} X_{\alpha}+\sum_{\alpha \in \Sigma_{\mathfrak{t}}^{+}} \boldsymbol{C} X_{\alpha}$. The proof of this is easy (cf. [W]). Hence it suffices for our purpose to determine $\Delta_{\mathfrak{i}}^{-}$. We note that $\mathfrak{f}_{s}$ is decomposed into at most two simple factors. Hence we may put $\mathfrak{f}_{s}=\mathfrak{f}_{s}^{1} \oplus \mathfrak{f}_{s}^{2}$, where each $\mathfrak{f}_{s}^{i}$ is simple or 0 . For convenience, we assume that rank $\mathfrak{f}_{s}^{1} \geqq \operatorname{rank} \mathfrak{f}_{s}^{2}$ and in particular that if $\mathfrak{l}_{s}$ is simple, then $\mathfrak{f}_{s}^{2}=0$. Moreover we put $\mathfrak{l}_{s}^{i}=\mathfrak{l} \cap \mathfrak{l}_{s}^{i}$. For simplicity, let $\Sigma_{i}$ be the root system of $\mathfrak{l}_{s}^{i}$ and put $\Delta_{i}=\Sigma_{i} \cap \Delta_{t}$, $\Delta_{i}^{-}=\Sigma_{i} \cap \Delta_{i}^{-}$.

We give here the explicit forms of $\Delta_{1}^{-}, \Delta_{2}^{-}$when $\mathfrak{g}$ is of classical type. The proof of them is easy and therefore is omitted. In the following we mainly discuss on the cases when $\mathfrak{l}_{s}$ is not a Borel subalgebra of $\mathfrak{f}_{s}$. If this is the case, it follows that $(\mathfrak{g}, \mathfrak{f})$ is one of the pairs $(\mathfrak{l l}(2 n, \boldsymbol{C}), \mathfrak{g} \mathfrak{p}(n, \boldsymbol{C})),(\mathfrak{g l}(m+n, \boldsymbol{C}), \mathfrak{g l}(m, \boldsymbol{C})+\mathfrak{g l}(n, \boldsymbol{C})+\boldsymbol{C}))$, $(\mathfrak{s p}(m+n, \boldsymbol{C}), \mathfrak{a p}(m, \boldsymbol{C})+\mathfrak{S p}(n, \boldsymbol{C}))$,

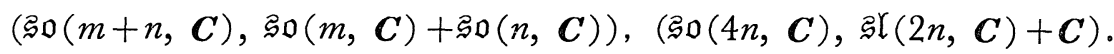

( I ) $(\mathfrak{a} \mathrm{l}(2 n, \boldsymbol{C}), \mathfrak{a p}(n, \boldsymbol{C}))$

We assume that $(\mathfrak{g}, \mathfrak{E})=(\mathfrak{g l}(2 n, \boldsymbol{C}), \mathfrak{g} \mathfrak{p}(n, \boldsymbol{C}))$. The root system of $\mathfrak{E}=\mathfrak{S p}(n, \boldsymbol{C})$ is then

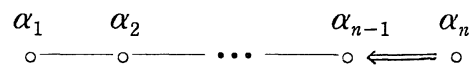


It follows that $\Delta_{\mathfrak{\mathrm { r }}}^{-}=\left\{\alpha_{2 i-1} ; i=1, \ldots,\left[\frac{n+1}{2}\right]\right\}$.

(II) $\quad(\mathfrak{I l}(m+n, \boldsymbol{C}), \mathfrak{I l}(m, \boldsymbol{C})+\mathfrak{I l}(n, \boldsymbol{C})+\boldsymbol{C}) \quad(m \geqq n \geqq 1)$

If $m-n \leqq 1$, then $\mathfrak{I}_{s}$ is a Borel subalgebra of $\mathfrak{f}_{s}$ (cf. Prop. 2.13).

Next we assume that $m \geqq n+2$. We note that $\mathfrak{I}_{s}^{2}$ is a Borel subalgebra of $\mathfrak{f}_{s}^{2}=\mathfrak{I} \mathfrak{l}(n, \boldsymbol{C})$. Since $\mathfrak{f}_{s}^{1}=\mathfrak{g l}(m, \boldsymbol{C})$, it follows that the root system $\Delta_{1}$ is

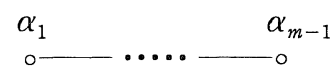

Using this, we find that $J_{1}^{-}=\left\{\alpha_{i} ;\left[\frac{n+1}{2}\right]<i<m-\left[\frac{n+1}{2}\right]\right\}$.

(III) $\quad(\mathfrak{S D}(m+n, \boldsymbol{C}), \mathfrak{S} \mathfrak{D}(m, \boldsymbol{C})+\mathfrak{S} \mathfrak{D}(n, \boldsymbol{C}))(m \geqq n \geqq 1)$

As in the previous case, if $m-n \leqq 1$, then $\mathfrak{l}_{s}$ is a Borel subalgebra of $\mathfrak{l}_{s}$.

We consider the case when $m \geqq n+2$. First assume that $n \geqq 3$. This implies that $\mathfrak{l}_{s}$ is not simple. Then we see that $\mathfrak{I}_{s}^{2}$ is a Borel subalgebra of $\mathfrak{f}_{s}^{2}=\mathfrak{I} \mathfrak{D}(n, \boldsymbol{C})$. In this case, $\mathfrak{f}_{s}^{1}=\mathfrak{g} \mathfrak{D}(m, \boldsymbol{C})$. Hence the root system $\Delta_{1}$ is different if $m$ is odd or even. For simplicity, we put $p=\left[\frac{m}{2}\right]$. If $m$ is odd, then $\Delta_{1}$ is

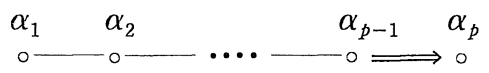

On the other hand, if $m$ is even, then $\Delta_{1}$ is

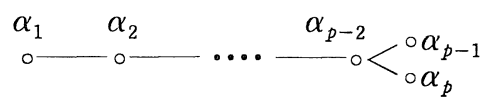

Using these, we obtain that $\Delta_{1}^{-}=\left\{\alpha_{i} ; i>\left[\frac{n+1}{2}\right]\right\}$.

Next consider the cases when $n=1$ and 2. In these cases, $\mathfrak{f}_{s}^{2}=0$, that is, $\mathfrak{f}_{s}$ is simple. But the results are similar to the above case. Namely, we have $\Delta_{1}^{-}=\left\{\alpha_{i} ; i>1\right\}$.

$$
\text { (IV) } \quad(\mathfrak{F p}(m+n, \boldsymbol{C}), \mathfrak{s p}(m, \boldsymbol{C})+\mathfrak{E p}(n, \boldsymbol{C}))(m \geqq n \geqq 1)
$$

If $m=n$, then $\mathfrak{l}_{s}$ is a Borel subalgebra of $\mathfrak{l}_{s}$.

We assume that $m \geqq n+1$. Since $\mathfrak{l}_{s}^{1}=\mathfrak{g p}(m, \boldsymbol{C})$ and $\mathfrak{f}_{s}^{2}=\mathfrak{F} \mathfrak{p}(n, \mathbb{C})$, we find that $\Delta_{1}$ is

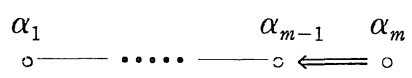

and $A_{2}$ is 


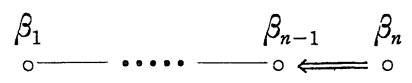

Then we find that $\Delta_{1}^{-}=\left\{\alpha_{2 i-1} ; i \leqq\left[\frac{n+1}{2}\right]\right\}$ and $\Delta_{2}^{-}=\left\{\beta_{2 i-1} ; 1 \leqq i \leqq\right.$ $\left.\left[\frac{n+1}{2}\right]\right\}$.

( V) $(\mathfrak{g v}(4 n, \boldsymbol{C}), \mathfrak{g l}(2 n, \boldsymbol{C})+\boldsymbol{C})$

In this case, $\mathfrak{l}_{s}=\mathfrak{g l}(2 n, \boldsymbol{C})$ is simple. The root system $\Delta_{\mathfrak{t}}$ is

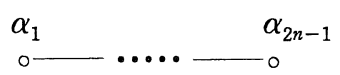

Then it follows that $\Delta_{i}^{-}=\left\{\alpha_{2 i} ; 1 \leqq i \leqq n-1\right\}$.

Remark 2.14. We do not mention the explicit form of $\Delta_{i}^{-}$when $(\mathfrak{g}, \mathfrak{f})$ is of exceptional type in this paper.

\section{§3. The Generic Singularities of the Nilpotent Subvariety The Case of Type $A$}

In the previous sections, we mainly examined the structure of $\mathscr{N}(V)_{\text {reg }}$ in some detail. In this section, we restrict our attention to such a pair $(\mathfrak{g}, \mathfrak{f})$ that $\mathfrak{g}$ is of type $A$ and examine the more fine structures of $\mathscr{N}(V)$.

First we propose a problem on the orbital structure of $\mathscr{N}(V)$.

Problem (3-I). Determine the $K_{\theta}$-orbits of $\mathscr{N}(V)$.

To this problem, Vinberg [V] gives an answer, generalizing Dynkin's method on the classification of the nilpotent orbits of a simple Lie algebra. But his result seems to be incomplete because of the lack of a concrete parametrization of the $K_{\theta}$-orbits analogous to the weighted Dynkin diagrams.

Secondly we interest on the determination of the closure relation.

Problem (3-II). Let $\mathcal{O}_{1}$ and $\mathcal{O}_{2}$ be $K_{\theta}$-orbits of $\mathscr{N}(V)$. Then give a condition to decide whether $\mathcal{O}_{1}$ is contained in the Zariski closure of $\mathrm{O}_{2}$ or not.

As for the nilpotent variety of a simple Lie algebra, this is already 
established by Gerstenhaber [G] and Hesselink [Hes 1] in the classical case and by Mizuno $[M 1,2]$ and others in the exceptional case.

Thirdly we restrict our attention to the generic singularities of $\mathscr{N}(V)$. Problems (3-III)-(3-V) below are based on the famous study of Brieskorn $[\mathrm{Br}]$ on the deep connection between the simple Lie algebras and the rational double points described as follows (see also $[\mathrm{Sl}])$ :

If $\mathrm{g}$ is a simple Lie algebra of $A l, D_{l}$ or $E_{l}$, and if $X$ is a subregular nilpotent element of $\mathfrak{g}$, then the intersection of a transversal slice of the orbit of $X$ with the nilpotent variety of $\mathfrak{g}$ realizes the rational double point of the corresponding type. Moreover the restriction of the Springer's resolution of the nilpotent variety to the inverse image of the intersection is identified with the minimal resolution of the rational double point in question. In particular the fibre of $X$ is a Dynkin curve. (For the definition of the Dynkin curve, see [St])。

Problem (3-III). Determine the $K_{\theta}$-orbits $\mathcal{O}_{1}, \ldots, \mathcal{O}_{r}$ of $\mathscr{N}(V)_{\text {sing }}$ with the conditions (a) and (b):

(a) each $\mathcal{O}_{i}$ is Zariski open in $\mathcal{N}(V)_{\text {sing }}$,

(b) the Zariski closure of the set $\mathscr{N}(V)_{\text {sing }}^{\prime}=\cup_{i=1}^{r} \mathcal{O}_{i}$ coincides with $\mathscr{N}(V)_{\text {sing. }}$.

Problem (3-IV). Let $X \in \mathscr{N}(V)_{\text {sing }}^{\prime}$ and let $\mathscr{S}_{X}$ be a transversal slice to the $K$-orbit of $X$ at $X$. Then determine the intersection $\overline{\mathscr{S}_{X}}=\mathscr{N}(V) \cap \mathscr{S}_{X}$.

Problem $(3-V)$. For any $X \in \mathcal{N}(V)_{\text {sing }}^{\prime}$, determine the fibre $\pi^{-1}(X)$ of $X$ in the resolution $\pi: \tilde{\mathscr{N}}(V) \rightarrow \mathscr{N}(V)$.

Remark. Problem (3-IV) is partly solved in [S-S].

In this section we consider such a symmetric pair $(\mathfrak{g}, \mathfrak{f})$ that $\mathfrak{g}$ is simple of type $A$ and obtain complete answers to Problems (3-I)$(3-\mathrm{V})$ for the pairs partly with the help of the work of Kempken [Ke].

We now give a proposition on a general symmetric pair. It plays an important role in the study on Problems (3-I)-(3-V). 
Proposition 3.1. Let $(\mathfrak{g}, \mathfrak{f})$ be a symmetric pair and let $d_{1}, \ldots$, $d_{l}$ be the degrees of the basic $K$-invariants. Assume as before that $d_{1} \leqq \ldots \leqq d_{l}$. Let $X$ be a nilpotent element of $V$ and let $(H, X, Y)$ be a corresponding normal $S$-triple. Now we use the notation in Proposition 1.24. If $\frac{1}{2} \tilde{\alpha}\left(H^{\prime}\right)+1<d_{l-1}$, then the 1 -forms $d P_{l-1}$ and $d P_{l}$ vanish at $X$.

Remark 3.2. The converse of Proposition 3.1 does not hold in general. A counterexample will be given in Example 4.9.

The proof of the proposition is similar to that of Lemma 1.23. Hence we omit it.

For later use, we prepare some notation.

(i) A $k$-tuple $\eta=\left(p_{1}, \ldots, p_{k}\right)$ of a partition of $n$ if $p_{1} \geqq \cdots \geqq$ $p_{k} \geqq 0$ and $p_{1}+\cdots+p_{k}=n$.

(ii) $P(n)$ is the set of partitions of $n$.

(iii) For any $\eta=\left(p_{1}, \ldots, p_{k}\right) \in P(n)$, we define a Jordan matrix

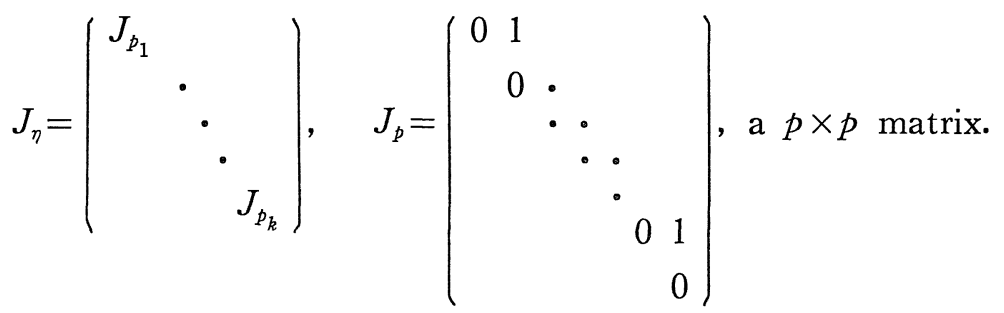

(iv) $I_{n}:$ the identity matrix of order $n$.

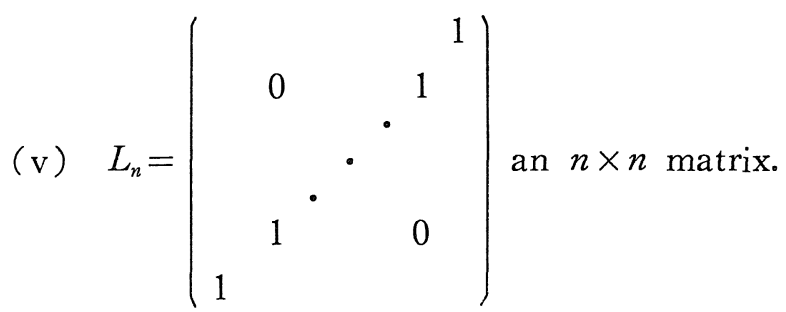

(vi) For any $X \in \mathfrak{g}, \mathcal{O}(X)$ (resp. $\mathcal{O}_{\theta}(X), O_{G}(X)$ ) denotes the $K$-(resp. $K_{\theta^{-}}, G^{-}$)orbit of $X$.

If $(\mathfrak{g}, \mathfrak{f})$ is a symmetric pair such that $\mathfrak{g}$ is of type $A$, then $(\mathfrak{g}, \mathfrak{f})$ is one of the pairs

(3.1) $(\mathfrak{s l}(n, \boldsymbol{C}), \mathfrak{S O}(n, \boldsymbol{C}))$, 
(3.2) $(\mathfrak{L l}(2 n, \boldsymbol{C}), \mathfrak{g} \mathfrak{p}(n, \boldsymbol{C}))$,

(3.3) $(\mathfrak{I l}(m+n, \boldsymbol{C}), \mathfrak{I l}(m, \boldsymbol{C})+\mathfrak{I l}(n, \boldsymbol{C})+\boldsymbol{C}) \quad(m \geqq n \geqq 1)$.

From now on we examine these pairs separately.

3. 1. The case of $(\mathfrak{I l}(n, \boldsymbol{C})$, $\mathfrak{B O}((n, \boldsymbol{C}))$

For the present, let $\mathfrak{g}$ denote $\mathfrak{g l}(n, \boldsymbol{C})$. We define an involution $\theta$ of $\mathfrak{g}$ by $\theta(X)=-L_{n}{ }^{t} X L_{n}$. Let $\mathfrak{g}=\mathfrak{i}+V$ be the direct sum decomposition corresponding to $\theta$. Then there is an element $g$ of $G L(n, \boldsymbol{C})$ such that $g^{\mathfrak{f}} g^{-1}=\left\{X \in \mathfrak{g} ;{ }^{t} X+X=0\right\}$ and $g V g^{-1}=\left\{X \in \mathfrak{g} ;{ }^{t} X=X\right\}$. We denote $\hat{G}=S L(n, C)$ and $\hat{K}=\left\{k \in \hat{G} ;{ }^{t} k L_{n} k=L_{n}\right\}$. Then $\hat{K}$ is connected and is isomorphic to $S O(n, C)$ and $\mathfrak{k}$ is the Lie algebra of $\hat{K}$. We denote $G=\hat{G} / Z$ and $K=\hat{K} / Z$, where $Z$ is the center of $\hat{G}$. It follows from Theorem 1 that if $n$ is odd, then $K_{\theta}=K$ and if $n$ is even, then $K_{\theta}$ has two connected components.

The followings are well-known facts and are easily checked.

(3. 1. a) If $n$ is odd, and if $\eta=\left(p_{1}, \ldots, p_{k}\right)$ is a partition of $n$, then $\mathcal{O}_{G}\left(J_{\eta}\right) \cap V$ is not empty and is a single $K$-orbit.

(3. 1. b) If $n$ is even and if $\eta=\left(p_{1}, \ldots, p_{k}\right) \quad\left(p_{1} \geqq \cdots \geqq p_{k}>0\right)$ is a partition of $n$, then $\mathcal{O}_{G}\left(J_{\eta}\right) \cap V$ is not empty and is a single $K_{\theta}$ orbit. Moreover, if at least one of $p_{1}, \ldots, p_{k}$ is odd, then $\mathcal{O}_{G}\left(J_{\eta}\right) \cap V$ becomes a single $K$-orbit but if all of $p_{1}, \ldots, p_{k}$ are even, then $\mathcal{O}_{G}\left(J_{\eta}\right) \cap V$ decomposes into just two $K$-orbits.

(3.1. c) Let $X$ be an element of $\mathcal{O}_{G}\left(J_{\eta}\right) \cap V$. Then we have $\operatorname{dim} Z_{\mathfrak{8}}(X) \cap V=\sum_{i=1}^{k} i p_{i}-1$, $\operatorname{dim} Z_{\mathfrak{g}}(X) \cap \mathfrak{t}=\sum_{i=1}^{k}(i-1) p_{i}$.

We introduce $K$-invariant polynomials $P_{2}, \cdots, P_{n}$ on $V$ by the following formula:

$$
\operatorname{det}\left(\lambda I_{n}+X\right)=\lambda^{n}+P_{2}(X) \lambda^{n-2}+\cdots+P_{n}(X) .
$$

Then it easily follows that $J=\boldsymbol{C}\left[P_{2}, \ldots, P_{n}\right]$. Using $P_{i}(2 \leqq i \leqq n)$, we define a map $\chi: V \rightarrow \boldsymbol{C}^{n-1}$ by $\chi(X)=\left(P_{2}(X), \ldots, P_{n}(X)\right)$.

Let $\mathscr{N}(V)$ be the nilpotent subvariety of $V$. Then

$$
\mathscr{N}(V)=\left\{X \in V ; P_{2}(X)=\cdots=P_{n}(X)=0\right\}
$$

and $\mathscr{N}(V)$ is the disjoint union of $\mathcal{O}_{G}\left(J_{\eta}\right) \cap V(\forall \eta \in P(n))$.

It follows from (3.1. c) that $X \in \mathscr{N}(V)$ is principal nilpotent if 
and only if $X$ is conjugate to $J_{n}$ through an element of $G$.

We next examine $\mathscr{N}(V)_{\text {sing. }}$. If $\eta=(n-1,1) \in P(n)$, then it follows from (3.1. a), (3.1. b) and (3.1. d) that $\mathcal{O}_{G}\left(J_{\eta}\right) \cap V$ is a single $K$-orbit and that $\mathscr{N}(V)_{\text {sing }}=\overline{\mathcal{O}_{G}\left(J_{\eta}\right) \cap V}$.

\section{Theorem 7.}

(a) $\mathscr{N}(V)_{\text {sing }}$ is the Zariski closure of a single $K$-orbit $\mathcal{O}$. Moreover, $\mathcal{O}=\mathcal{O}_{G}\left(J_{\eta}\right) \cap V(\eta=(n-1,1))$.

(b) Fix a nilpotent element $X_{0}$ of $\mathcal{O}$ and its transversal slice $\mathscr{S}_{X_{0}}$ Put $\delta=\chi \mid \mathscr{S}_{X_{0}}$. Then for any $\xi=\left(\xi_{2}, \ldots, \xi_{n}\right) \in C^{n-1}, \delta^{-1}(\xi)$ is biholomorphic to the curve

$$
C_{\xi}=\left\{(x, y) \in C^{2} ; x^{n}+y^{2}+\xi_{2} x^{n-2}+\cdots+\xi_{n}=0\right\} .
$$

In particular, $\delta^{-1}(0)=\mathscr{S}_{x_{0}} \cap \mathscr{N}(V)$ is biholomorphic to $\left\{(x, y) \in \boldsymbol{C}^{2} ; x^{n}\right.$ $\left.+y^{2}=0\right\}$. (This curve is regarded as two-dimensional $A_{n-1}$-singularity.)

(c) If $n$ is odd, then $\pi^{-1}\left(\mathscr{S}_{X_{0}} \cap \mathscr{N}(V)\right)$ is an affine line and if $n$ is even, then $\pi^{-1}\left(\mathscr{S}_{X_{0}} \cap \mathscr{N}(V)\right)$ is a disjoint union of two affine lines. Hence $\pi: \pi^{-1}\left(\mathscr{S}_{X_{0}} \cap \mathscr{N}(V)\right) \rightarrow \mathscr{S}_{X_{0}} \cap \mathcal{N}(V)$ realizes the normalization of two-dimensional $A_{n-1}$-singularity.

Proof. The claim (a) follows from the remark before the theorem.

To prove (b), we take out a special nilpotent element $X_{0}$ of $V$ with its transversal slice. If $n$ is odd, then $X_{0}=J_{n}$ and if $n$ is even,

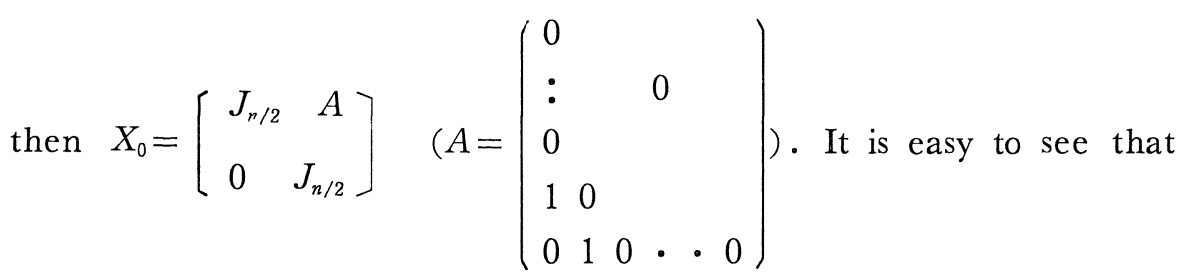
$X_{0}$ is contained in $V$ and its Jordan's normal form is $J_{(n-1,1)}$. Furthermore, we set

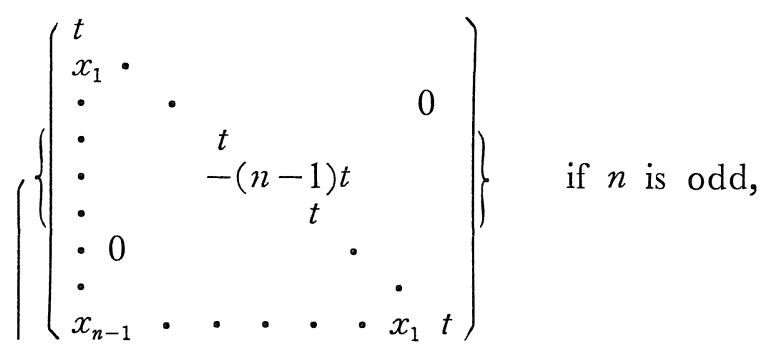




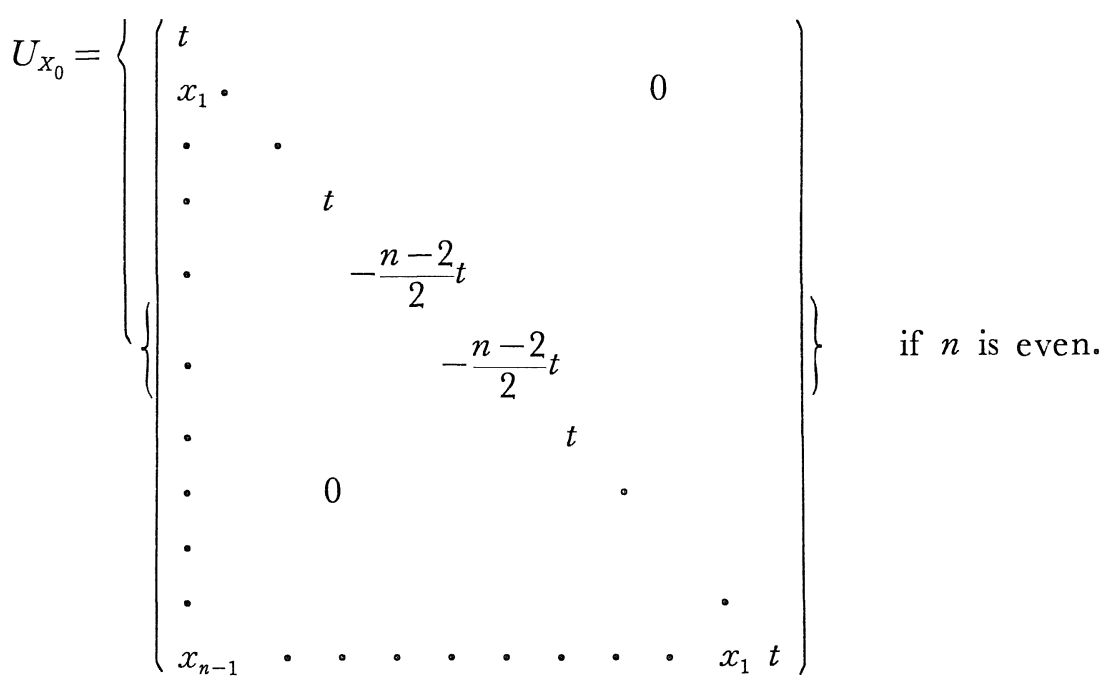

By direct calculation, we see that $\mathscr{S}_{X_{0}}=X_{0}+U_{X_{0}}$ is a transversal slice to the $K$-orbit of $X_{0}$. We consider the equation

$$
\operatorname{det}\left(\lambda I_{n}+X\right)=\lambda^{n}+\sum_{i=2}^{n} \xi_{i} \lambda^{n-i} \quad\left(X \in \mathscr{S}_{X_{0}}\right) .
$$

From this we conclude that

$$
\delta^{-1}(\xi)=\left\{(x, y) \in C^{2} ; x^{n}+y^{2}+\sum_{i=2}^{n} \xi_{i} x^{n-i}=0\right\}
$$

for any $\xi \in \boldsymbol{C}^{n-1}$. Hence (b) is proved.

Last we prove (c). If $n$ is odd, it follows that $\mathcal{N}(V)$ is irreducible. Hence Proposition 2.7 implies that $\pi^{-1}\left(\mathscr{S}_{X_{0}} \cap \mathscr{N}(V)\right)$ is a non-singular line. On the other hand, if $n=2 m$, even, then $\mathscr{S}_{X_{0}} \cap \mathcal{N}(V)=\{(x, y)$; $\left.x^{2 m}+y^{2}=0\right\}$ has two connected components $D_{1}=\left\{x^{m}+\sqrt{-1} y=0\right\}$ and $D_{2}=\left\{x^{m}-\sqrt{-1} y=0\right\}$. Hence Proposition 2.7 implies that $\pi^{-1}\left(D_{i}\right)(i=$ $1,2)$ is a non-singular line. But clearly $\pi^{-1}\left(\mathscr{S}_{x_{0}} \cap \mathscr{N}(V)\right)$ is the disjoint union of $\pi^{-1}\left(D_{1}\right)$ and $\pi^{-1}\left(D_{2}\right)$. We have thus proved (c). q.e.d.

\section{2. The case of $(\mathfrak{I l}(2 n, \boldsymbol{C}), \mathfrak{g p}(n, \boldsymbol{C}))$}

We next set $\mathfrak{g}=\mathfrak{g l}(2 n, \boldsymbol{C})$ and define an involution $\theta$ of $\mathfrak{g}$ by $\theta(X)=\left(\begin{array}{cc}0 & L_{n} \\ -L_{n} & 0\end{array}\right)^{t} X\left(\begin{array}{cc}0 & L_{n} \\ -L_{n} & 0\end{array}\right)(X \in \mathfrak{g})$. Let $\mathfrak{g}=\mathfrak{f}+V$ be the direct 
sum decomposition of $\mathfrak{g}$ corresponding to $\theta$. Then clearly $\mathfrak{i}$ is a subalgebra of $\mathfrak{g}$ isomorphic to $\mathfrak{s p}(n, \boldsymbol{C})$. We also let $G$ denote $S L(2 n, \boldsymbol{C})$, where $K$ denotes the analytic subgroup of $G$ corresponding to f. In this case, clearly $K_{\theta}=K$.

For any $\eta=\left(p_{1}, p_{2}, \ldots, p_{k}\right) \in P(n)$, define $\eta^{2}=\left(p_{1}, p_{1}, p_{2}, p_{2}, \ldots\right.$, $\left.p_{k}, p_{k}\right)$. Then $\eta^{2}$ is contained in $P(2 n)$. For convenience, we set $P^{\prime}(2 n)=\left\{\eta^{2} ; \eta \in P(n)\right\}$.

We now give some elementary properties of nilpotent elements contained in $\mathscr{N}(V)$ similar to (3.1. a)-(3.1. d).

(3.2. a) For any $\nu \in P(2 n), A d(G) J_{\nu} \cap V \neq \phi$ if and only if $\nu$ is contained in $P^{\prime}(2 n)$. Moreover, if $\nu$ is contained in $P^{\prime}(2 n)$, then $A d(G) J_{\nu} \cap V$ is a single $K$-orbit.

Proof. Since $\left[\begin{array}{cc}X & 0 \\ 0 & L_{n}^{t} X L_{n}\end{array}\right] \quad(X \in \mathfrak{b l}(n, C))$ is contained in $V$, $A d(G) J_{\nu} \cap V$ is not empty if $\nu$ is contained in $P^{\prime}(2 n)$. Next we show that if an element $X$ of $\mathfrak{g}$ is contained in $\mathcal{N}(V)$ and if $J_{\nu}(\nu \in P(2 n))$ is the Jordan's normal form of $X$, then $\nu$ is contained in $P^{\prime}(2 n)$. Let $(H, X, Y)$ be a normal $S$-triple corresponding to $X$. Define $H^{\prime}, X^{\prime}$, $Y^{\prime}$ as in (1.1). Then by definition, $X$ and $X^{\prime}$ are $G$-conjugate. We find that $H^{\prime}$ is contained in $V$ and therefore is conjugate to a diagonal matrix diag $\left(t_{1}, \ldots, t_{n}, t_{n}, \ldots, t_{1}\right)$ contained in $V$. In terms of $t_{1}, \ldots, t_{n}$, the partition $\nu$ of $2 n$ is uniquely determined, and from this we easily conclude that $\nu$ is contained in $P^{\prime}(2 n)$.

If $A$ and $B$ are diagonal matrices contained in $\mathfrak{f}$ whose sets of the eigenvalues coincide, there is an element $k$ of $K$ such that $k A k^{-1}$ $=B$. Noting this, we conclude from Lemma 1.2 and the uniqueness cf the conjugacy class of Cartan subalgebras of a complex semisimple Lie algebra that $A d(G) J_{\nu} \cap V$ is a single $K$-orbit. q. e.d.

(3. 2. b) Let $\eta=\left(p_{1}, \ldots, p_{k}\right)$ be a partition of $n$ and put $\nu=\eta^{2}$. Let $X$ be an element of $A d(G) J_{\nu} \cap V$. Then

$$
\begin{aligned}
& \operatorname{dim} Z_{\mathfrak{g}}(X) \cap V=\sum_{i=1}^{k}(4 i-3) p_{i}-1, \\
& \operatorname{dim} Z_{\mathfrak{g}}(X) \cap \mathfrak{t}=\sum_{i=1}^{k}(4 i-1) p_{i} .
\end{aligned}
$$


(3.2. c) For any two partitions $\eta, \nu \in P^{\prime}(2 n), \quad A d(G) J_{\nu} \cap V$ is contained in $\overline{A d(G) J_{n} \cap V}$ if and only if $A d(G) J_{\nu}$ is contained in $\overline{\operatorname{Ad}(G) J_{\eta}}$.

The claims (3.2. b) and (3.2. c) are easily checked. Hence we omit the proof.

As in 3.1 , we define polynomials $P_{2}(X), \ldots, P_{n}(X)$ on $V$ by the formula

$$
\operatorname{det}\left(\lambda I_{n}+X\right)=\left(\lambda^{n}+P_{2}(X) \lambda^{n-2}+\cdots+P_{n}(X)\right)^{2} \quad(X \in V) .
$$

Then clearly $J=\boldsymbol{C}\left[P_{2}, \ldots, P_{n}\right]$. Using $P_{2}, \ldots, P_{n}$, we define $\chi: V$ $\rightarrow \boldsymbol{C}^{n-1}$ by $\chi(X)=\left(P_{2}(X), \ldots, P_{n}(X)\right)$.

Let $\mathcal{N}(V)$ be the nilpotent subvariety of $V$. Then an element $X$ of $\mathscr{N}(V)$ is principal nilpotent if and only if $J_{(n, n)}$ is the Jordan's normal form of $X$. This follows from (3.2. b). By definition, $J_{(n, n)}$ is contained in $V$. Now fix $X=J_{(n, n)}$ and denote by $(H, X, Y)$ its corresponding normal $S$-triple. We can take $H$ as a diagonal matrix. Then $H, Y$ are uniquely determined. By using the $S$-triple, we define $\mathfrak{l}, L$ and $\mathfrak{n}$ as we $\operatorname{did}$ in $\S 2$. Then $\tilde{\mathscr{N}}(V)=K \times{ }^{L} \mathfrak{n}$ is the desingularization of $\tilde{\mathscr{N}}(V)$ (cf. $\S 2$ ). Let us put

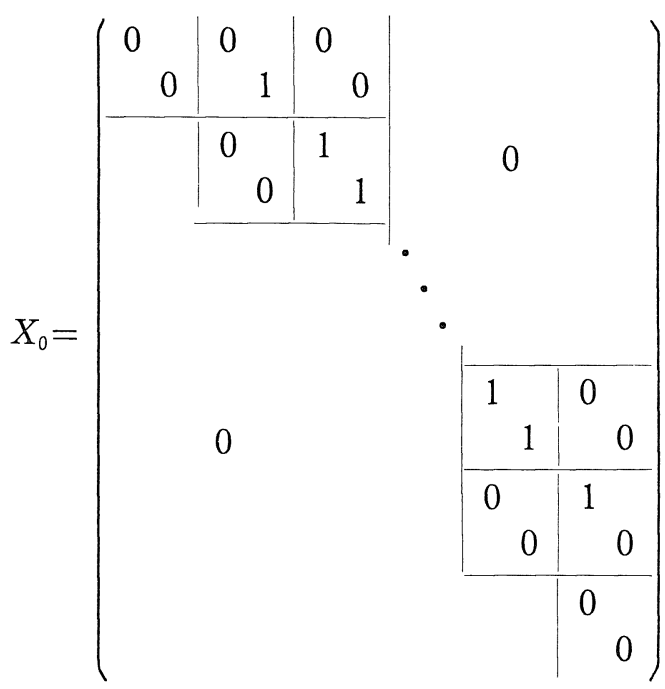

and let $\mathcal{O}$ be the $K$-orbit cf $X_{0}$. Then (3.2. c) implies that $\mathscr{N}(V)_{\text {sing }}$ is the Zariski closure of $\mathcal{O}$. Let $\mathscr{S}_{X_{0}}$ be the transversal slice to $\mathcal{O}$ at $X_{0}$ defined as in Lemma 1.21. Then we obtain the following. 


\section{Theorem 8.}

(a) $\mathscr{N}(V)_{\text {sing }}$ is the Zariski closure of the single $K$-orbit $\mathcal{O}=$ $A d(K) X_{0}$.

(b) Put $\delta=\chi \mid \mathscr{S}_{X_{0}}$. Then for any $\xi=\left(\xi_{2}, \ldots, \xi_{n}\right) \in \boldsymbol{C}^{n-1}, \delta^{-1}(\xi)$ is biholomorphic to the hypersurface

$$
\begin{aligned}
\left\{\left(x, y_{1}, y_{2}, y_{3}, y_{4}\right) \in \boldsymbol{C}^{5} ;\right. & x^{n}+y_{1}^{2}+y_{2}^{2}+y_{3}^{2}+y_{4}^{2} \\
& \left.+\xi_{2} x^{n-2}+\cdots+\xi_{n-1} x+\xi_{n}=0\right\} .
\end{aligned}
$$

In particular, $\delta^{-1}(0)=\mathscr{S}_{X_{0}} \cap \mathcal{N}(V)$ is biholomorphic to the hypersurface $\left\{\left(x, y_{1}, y_{2}, y_{3}, y_{4}\right) \in \boldsymbol{C}^{5} ; x^{n}+y_{1}^{2}+y_{2}^{2}+y_{3}^{2}+y_{4}^{2}=0\right\}$.

(c) $\tilde{\mathscr{S}}=\pi^{-1}\left(\mathscr{S}_{X_{0}} \cap \mathscr{N}(V)\right)$ is non-singular and $\pi$ induces a resolution $\tilde{\mathscr{S}} \rightarrow \mathscr{S}_{X_{0}} \cap \mathscr{N}(V)$.

(d) Let us define the varieties $E, F, \tilde{F}$ :

$$
\begin{aligned}
E= & \left\{\left(\xi_{0}: \xi_{1}: \xi_{2}: \xi_{3}: \xi_{4}\right) \in \boldsymbol{P}^{4} ; \xi_{0}^{2}=\xi_{1} \xi_{4}-\xi_{2} \xi_{3}\right\}, \\
F= & \left\{\left(\eta_{0}: \eta_{1}: \eta_{2}: \eta_{3}: \eta_{4}\right) \in \boldsymbol{P}^{4} ; \eta_{1} \eta_{4}-\eta_{2} \eta_{3}=0\right\}, \\
\widetilde{F}= & \left\{\left(\left(\eta_{0}: \eta_{1}: \eta_{2}: \eta_{3}: \eta_{4}\right),\left(\zeta_{1}: \zeta_{2}: \zeta_{3}: \zeta_{4}\right)\right) \in \boldsymbol{P}^{4} \times \boldsymbol{P}^{3} ;\right. \\
& \left.\eta_{1} \eta_{4}-\eta_{2} \eta_{3}=\zeta_{1} \zeta_{4}-\zeta_{2} \zeta_{3}=0,\left(\eta_{1}, \eta_{2}, \eta_{3}, \eta_{4}\right) / /\left(\zeta_{1}, \zeta_{2}, \zeta_{3}, \zeta_{4}\right)\right\} .
\end{aligned}
$$

Then for any $X \in \mathcal{O}$, we have $\pi^{-1}(X)=\cup_{i=1}^{m} D_{i}\left(m=\left[\frac{n}{2}\right]\right)$, where $D_{1}$ is biholomorphic to $E$ (resp. $F$ ) if $n$ is even (resp. odd), and $D_{\imath}(2 \leqq i \leqq$ $m$ ) is to $\widetilde{F}$, and moreover

$$
D_{i} \cap D_{j}= \begin{cases}\boldsymbol{P}^{1} \times \boldsymbol{P}^{1} & (i-j= \pm 1) \\ \phi & (i-j \neq \pm 1)\end{cases}
$$

Proof. The claim (a) follows from the discussion before the theorem. Next we consider the equation

$$
\operatorname{det}\left(\lambda I_{n}+X\right)=\left(\lambda^{n}+\sum_{i=2}^{n} \xi_{i} \lambda^{n-i}\right)^{2} \quad\left(X \in \mathscr{S}_{X_{0}}\right) .
$$

Then by direct calculation, we conclude the claim (b). The claim (c) is an easy consequence of Proposition 2.7.

We now show (d). By (a), we may take $X=X_{0}$ without loss of generality. Let us first assume that $n$ is even, i. e., $n=2 m$. In order to describe $\pi^{-1}(X)$, we define the matrices $w_{i}(0 \leqq i \leqq n-1), k_{d}^{\prime}(x, y$, z) $(1 \leqq d \leqq m)$ as follows: 


$$
\begin{aligned}
& w_{i}=\left(\begin{array}{cc}
w_{i}^{\prime} & 0 \\
0 & w_{n-i}^{\prime}
\end{array}\right) \quad\left(w_{i}^{\prime}=\left(\begin{array}{cccc}
I_{i-1} & & \\
& 0 & 1 & \\
& 1 & 0 & \\
& & & I_{n-i-1}
\end{array}\right)\right) \quad(1 \leqq i \leqq n-1) \\
& k_{1}^{\prime}(x, y, z)=\left(\begin{array}{llllllll}
I_{n-2} & & & & & 0 & \\
0 & 1 & & & & \\
0 & 0 & 1 & & & & \\
0 & x & y & 1 & & & \\
0 & z & x & 0 & 1 & & \\
0 & 0 & 0 & 0 & 0 & I_{n-2}
\end{array}\right)
\end{aligned}
$$

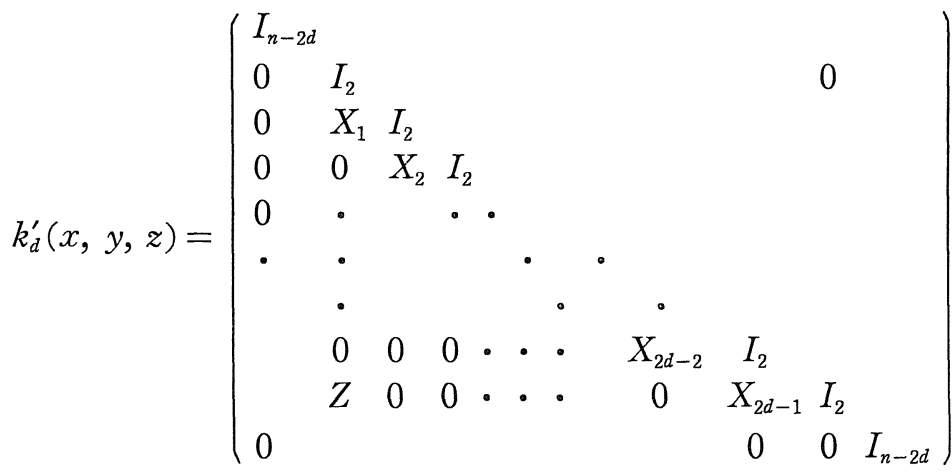

$$
\begin{aligned}
& X_{1}=\left(\begin{array}{cc}
x & y \\
0 & 0
\end{array}\right), \quad X_{2 d-1}=\left(\begin{array}{cc}
0 & -y \\
0 & -x
\end{array}\right), \quad Z=\left(\begin{array}{cc}
0 & 0 \\
z & 0
\end{array}\right), \\
& X_{2}=\cdots=X_{d}=-X_{d+1}=\cdots=-X_{2 d-2}=\left(\begin{array}{ll}
0 & y \\
0 & 0
\end{array}\right) \text {. }
\end{aligned}
$$

On the other hand, as noted before, if $\mathfrak{p}$ is the parabolic algebra of $\mathfrak{g}$ consisting of the matrices of the form

$$
\left(\begin{array}{ccccccc}
X_{11} & X_{12} & X_{13} & \bullet & \bullet & \bullet & X_{1, n} \\
0 & X_{22} & X_{23} & \bullet & \bullet & \bullet & X_{2, n} \\
0 & 0 & X_{33} & \bullet & \bullet & \bullet & X_{3, n} \\
\bullet & \bullet & \bullet & \bullet & & & \bullet \\
\bullet & \bullet & \bullet & & \bullet & & \bullet \\
\bullet & \bullet & \bullet & & & \bullet & \bullet \\
0 & 0 & 0 & \bullet & \bullet & \bullet & X_{n, n}
\end{array}\right)
$$

(Each $X_{i j}$ is a $2 \times 2$ matrix.)

and $\tilde{\mathfrak{n}}$ is the nilpotent radical of $\mathfrak{p}$, then it follows that $\mathfrak{l}=\mathfrak{p} \cap \mathfrak{f}$ and $\mathfrak{n}=\tilde{\mathfrak{n}} \cap V . \quad$ By definition, $w_{i}(0 \leqq i \leqq n-1)$ and $k_{d}^{\prime}(x, y, z) \quad(1 \leqq d \leqq m$, 
$x, y, z \in \boldsymbol{C})$ are contained in $K$. Put $k_{d}(x, y, z)=w_{1} w_{2} \cdots w_{2 m-2 d}$ $k_{d}^{\prime}(x, y, z)$ and $Y_{d}(x, y, z)=k_{d}^{-1} X k_{d}$ for $d=1, \ldots, m$. Then it is easy to see that $Y_{d}(x, y, z)$ is contained in $\mathfrak{n}$ for any $x, y, z \in \boldsymbol{C}$ and $1 \leqq$ $d \leqq m$. From this remark it follows that $k_{d}(x, y, z) * Y_{d}(x, y, z)$ is contained in $\pi^{-1}(X)$ for any $x, y, z \in \boldsymbol{C}$ and $d$. Let $D_{d}$ be the Zariski closure of the set $\left\{k_{d} * Y_{d} \in \mathscr{N}(V) ; x, y, z \in \boldsymbol{C}\right\}$. Then each $D_{d}$ is contained in $\pi^{-1}(X)$. We now determine the explicit form of $D_{1}, \ldots$, $D_{m}$ by using a finite dimensional representation of $K$ with a $L$-fixed vector. Let $e_{i}$ be the column vector of size $2 n$ whose $i$-th entry is 1 and others are zero. We note that $K$ acts on each $e_{i}$ from the left naturally. Using these vectors, we define $v_{0}=e_{1} \wedge e_{2} \otimes e_{1} \wedge e_{2} \wedge e_{3} \wedge e_{4} \otimes \cdots$ $\otimes e_{1} \wedge e_{2} \wedge \cdots \wedge e_{n-1} \wedge e_{n}$. Then it follows that $L$ coincides with the set $\left\{k \in K ; k v_{0} \in \boldsymbol{C} v_{0}\right\}$. Let $U$ be the vector space spanned by the linear combinations of the vectors $k v_{0}(k \in K)$. Then we obtain a finite dimensional irreducible representation of $K$ on $U$. Let $\boldsymbol{P}(U)$ be the projective space associated with $U$, that is, $\boldsymbol{P}(U)$ consists of all lines $[v]=\boldsymbol{C} v(v \in U-\{0\})$. Then $K / L$ is isomorphic to the $K$-orbit $\left\{\left[k v_{0}\right] \in \boldsymbol{P}(U) ; k \in K\right\}$ of $\boldsymbol{P}(U)$. We note here that the map $D_{d} \ni$ $k * Y \rightarrow k L \in K / L$ is injective for any $d$. Accordingly, to determine $D_{d}$, we have only to calculate the Zariski closure of the set $\left\{\left[k_{d}(x, y, z) v_{0}\right]\right.$; $x, y, z \in \boldsymbol{C}\}$. For convenience, we identify this set with $D_{d}$. Then by an elementary computation, we conclude that $D_{1}=E$ and $D_{d}=\tilde{F}$ $(2 \leqq d \leqq m)$. We now determine $D_{d-1} \cap D_{d}$ for $d=2, \ldots, m$. Since $\lim _{x \rightarrow \infty}\left[k_{d}(x, y, z) v_{0}\right]=\left[k_{d-1}(0, y, z) v_{0}\right]$ and since $D_{d-1}$ and $D_{d}$ do not coincide, it follows that $\left\{\left[k_{d-1}(0, y, z) v_{0}\right] ; y, z \in \boldsymbol{C}\right\}$ is open dense in $D_{d-1} \cap D_{d}$. The Zariski closure of this set is isomorphic to $\boldsymbol{P}^{1} \times \boldsymbol{P}^{1}$. Hence we arrive at the conclusion that $D_{d-1} \cap D_{d}=\boldsymbol{P}^{1} \times \boldsymbol{P}^{1}$. On the other hand, from the definition, we find that $D_{i} \cap D_{j}=\phi$ if $i-j \neq \pm 1$. In view of the definition, we have only to show that if $k \in K$ satisfies the condition $k^{-1} X k \in \mathfrak{n}$, then $k *\left(k^{-1} X k\right) \in \bigcup_{i=1}^{m} D_{i}$. But this is provable by a straightforward calculation. Hence if $n$ is even, then (d) is completely proved. The proof of (d) in the case when $n$ is odd goes similarly to that in the even case, so we omit it.

Remark 3.3. Steinberg [St, 3.10, Th. 2] put forward various characterizations of subregular nilpotent elements of a simple Lie algebra. In particular a nilpotent element of a simple Lie algebra is 
subregular if and only if the variety of Borel subalgebras containing it is a Dynkin curve. Theorem 8 (d) is an analogue to this result for the vector space $V$ associated to the symmetric pair $(\mathfrak{I l}(2 n, \boldsymbol{C})$, $\mathfrak{g p}(n, \boldsymbol{C}))$.

Remark 3.4. A natural resolution of the hypersurface

$$
S_{N, n}=\left\{(x, y) \in C^{1+N} ; x^{n}+<y, y>=0\right\}
$$

in the affine space $\boldsymbol{C}^{1+N}(N \geqq 3)$ is constructed in the following manner. (Here we used the notation $y=\left(y_{1}, \ldots, y_{N}\right)$ and $\langle y, y\rangle=y_{1}^{2}+\cdots+$ $y_{N}^{2}$.) Put $m=\left[\frac{n}{2}\right]$ and define

$$
\begin{aligned}
\tilde{S}_{N . n}=\{((x, y), & \left.\left(\xi^{(1)}: \eta^{(1)}\right), \ldots,\left(\xi^{(m)}: \eta^{(m)}\right)\right) \in S_{N n} \times\left(\mathbb{P}^{N}\right)^{m} ; \\
& (x, y) \in\left(\xi^{(1)}: \eta^{(1)}\right), \\
& \left(\xi^{(i-1)} x, \eta^{(i-1)}\right) \in\left(\xi^{(i)}: \eta^{(i)}\right) \quad(i>1), \\
& \left.x^{n-2 i}\left(\xi^{(i)}\right)^{2}+<\eta^{(i)}, \eta^{(i)}>=0(\forall i)\right\}
\end{aligned}
$$

and $p: \tilde{S}_{N, n} \rightarrow S_{N, n}$ is the natural projection. Then we find that $p:$ $\tilde{S}_{N, n} \rightarrow S_{N, n}$ is a resolution of $S_{N . n}$ if $n \geqq 2$. In particular, the following conditions hold:

(a) $\tilde{S}_{N, n}$ is non-singular,

(b) $p$ induces a bijection $p^{-1}\left(S_{N, n}-\{0\}\right) \rightarrow S_{N, n}-\{0\}$,

(c) $p^{-1}(0)$ has $m$ number of irreducible components $B_{1}, \ldots, B_{m}$, where

$$
\begin{aligned}
& B_{i}=\left\{\left(\left(\xi: \eta_{1}: \cdots: \eta_{N}\right),\left(\zeta_{1}: \cdots: \zeta_{N}\right)\right) \in \mathbb{P}^{N} \times \mathbb{P}^{N-1}\right. \text {; } \\
& \eta_{1}^{2}+\cdots+\eta_{N}^{2}=\zeta_{1}^{2}+\cdots+\zeta_{N}^{2}=0 \\
& \left.\left(\eta_{1}, \ldots, \eta_{N}\right) / /\left(\zeta_{1}, \ldots, \zeta_{N}\right)\right\} \quad(1 \leqq i \leqq m-1), \\
& B_{m}=\left\{\begin{array}{l}
\left\{\left(\xi: \eta_{1}: \cdots: \eta_{N}\right) \in \boldsymbol{P}^{N} ; \xi^{2}+\eta_{1}^{2}+\cdots+\eta_{N}^{2}=0\right\} \text { if } n \text { is even } \\
\left\{\left(\xi: \eta_{1}: \cdots: \eta_{N}\right) \in \mathbb{P}^{N} ; \eta_{1}^{2}+\cdots+\eta_{N}^{2}=0\right\} \text { if } n \text { is odd, }
\end{array}\right.
\end{aligned}
$$

and

$$
B_{i} \cap B_{j}=\left\{\begin{array}{cc}
\left\{\left(\eta_{1}: \cdots: \eta_{N}\right) \in \boldsymbol{P}^{N-1} ; \eta_{1}^{2}+\cdots+\eta_{N}^{2}=0\right\} & (i-j= \pm 1) \\
\phi & (i-j \neq \pm 1) .
\end{array}\right.
$$

Theorem 8 (b) claims that $\mathscr{S}_{X_{0}} \cap \mathscr{N}(V)$ is identfied with $S_{4, n}$ defined above and Theorem 8(d) and (c) imply that the exceptional fibre as well as the configurations of irreducible components of it coincide for the two resolutions of the hypersurface $S_{4 n}$, one is obtained by using the group theory as in Theorem 8 and the other is constructed above. Hence we may conjecture 
Conjecture. Under the above notation, $\tilde{\mathscr{S}}$ is biholomorphic to $\tilde{S_{4 . n}}$.

If $n \leqq 4$, this conjecture is proved by a straightforward calculation.

\subsection{The case of $(\mathfrak{I l}(m+n, \boldsymbol{C}), \mathfrak{g l}(m, \boldsymbol{C})+\mathfrak{I l}(n, \boldsymbol{C})+\boldsymbol{C})(m \geqq$ $n \geqq 1$ )}

Noting that the pair in question is regarded as an example of vector space crowns, we find that Problems (3-I)-(3-IV) are already solved by Kempken [Ke].

For later use, we introduce the "diagrams" in order to parametrize the $K$-orbits of $\mathscr{N}(V)$. Let $w=\left(\varepsilon_{1} \ldots \varepsilon_{p}\right)$ be a $p$-tuple of numbers $\varepsilon_{1}, \ldots, \varepsilon_{p}$ such that $\varepsilon_{2 i-1}=1$ and $\varepsilon_{2 i}=2(\forall i)$ or that $\varepsilon_{2 i-1}=2$ and $\varepsilon_{2 i}=1$ $(\forall i)$. Then $w$ is called a word and $|w|=p$ is the length of $w$. If $w_{1}, \ldots, w_{r}$ are words, then $D=\left|\begin{array}{c}w_{1} \\ \vdots \\ w_{r}\end{array}\right|$ is called a diagram. Let $w_{1}=$ $\left(\varepsilon_{1} \ldots \varepsilon_{p}\right)$ and $w_{2}=\left(\delta_{1} \ldots \delta_{q}\right)$ be words. If $\varepsilon_{p} \neq \delta_{1}$, then we define a word $w_{1} w_{2}=\left(\varepsilon_{1} \ldots \varepsilon_{p} \delta_{1} \ldots \delta_{q}\right)$.

We put $\mathfrak{g}=\mathfrak{g l}(m+n, \boldsymbol{C})$ and define an involution $\theta$ of $\mathfrak{g}$ by $\theta(Z)=\left(\begin{array}{cc}I_{m} & 0 \\ 0 & -I_{n}\end{array}\right) Z\left(\begin{array}{cc}I_{m} & 0 \\ 0 & -I_{n}\end{array}\right) . \quad$ Then $\mathfrak{f}=\left\{\left(\begin{array}{cc}A & 0 \\ 0 & B\end{array}\right) ; A \in \mathfrak{g} \mathfrak{l}(m, C)\right.$, $B \in \operatorname{gl}(n, \boldsymbol{C}), \operatorname{Tr} A+\operatorname{Tr} B=0\}$ and $V=\left\{\left(\begin{array}{cc}0 & X \\ Y & 0\end{array}\right) ; X\right.$ is a $m \times n$ matrix and $Y$ is a $n \times m$ matrix $\}$. Let $U=\left\{x=\left(\begin{array}{c}x_{1} \\ \vdots \\ x_{m+n}\end{array}\right) ; x_{i} \in \boldsymbol{C}\right\}=\boldsymbol{C}^{m+n}$ and let $U_{1}=\left\{x \in U ; x_{m+1}=\cdots=x_{m+n}=0\right\}$ and $U_{2}=\left\{x \in U ; x_{1}=\cdots=x_{m}=\right.$ $0\}$. Now assume that $Z$ is a nilpotent element of $V$, i. e., $Z^{p}=0$ for some integer $p>0$. Let $J_{\eta}$ be the Jordan's normal form of $Z$, where $\eta=\left(p_{1}, \ldots, p_{r}\right) \in P(m+n), p_{1} \geqq \cdots \geqq p_{r}>0$. Then there exist vectors $v^{(1)}, \ldots, v^{(r)}$ of $U$ with the conditions (1)-(3):

(1) For any $i, v^{(i)}$ is contained in $U_{1}$ or $U_{2}$.

(2) Define $v_{j}^{(i)}=Z^{j-1} v^{(i)}(j=1,2, \ldots)$. Then $v_{p_{i}}^{(i)} \neq 0$ and $v_{p_{i}+1}^{(i)}$ $=0$.

(3) $\quad\left\{v_{j}^{(i)} ; 1 \leqq i \leqq r, 1 \leqq j \leqq p_{i}\right\}$ is a basis of $U$.

It follows from the definition that for any $i$ and $j, v_{j}^{(i)}$ is contained in $U_{1}$ or $U_{2}$. Noting this, we define a word $w_{i}=\left(\varepsilon_{1}^{(i)} \ldots \varepsilon_{p_{i}}^{(i)}\right)$, where 
$\varepsilon_{j}^{(i)}=d$ if $v_{j}^{(i)}$ is contained in $U_{d}(d=1,2)$. Hence we can define a diagram $D_{Z}=\left|\begin{array}{c}w_{1} \\ \vdots \\ w_{r}\end{array}\right|$, which is called the diagram of $Z$. The following lemma is stated in [Ke].

Lemma 3.5. Let $Z$ and $Z$ be nilpotent elements of $V$. Then $Z$ and $Z^{\prime}$ are $K$-conjugate if and only if $D_{Z}$ and $D_{Z^{\prime}}$ are equal up to permutation of the words.

We introduce the notion of a minimal degeneration. Let $Z$ and $Z^{\prime}$ be nilpotent elements of $V$. We write $D_{Z^{\prime}}<D_{Z}$ if $K Z^{\prime} \subset \overline{K Z}$. If $D_{Z^{\prime}}<D_{Z}$ and $K Z^{\prime}$ is open in $\overline{K Z}-K Z$, we call $K Z^{\prime} \subset \overline{K Z}$ (resp. $D_{Z^{\prime}}<$ $\left.D_{Z}\right)$ a minimal degeneration. As for a minimal degeneration, the following lemma is known.

Lemma 3.6 ([Ke]). If $D_{Z^{\prime}}<D_{Z}$ is a minimal degeneration, then the diagram of $Z$ is obtained from the diagram of $Z$ by replacing a pair of word $w^{\prime}$ of $D_{Z}$ of the form $w=w_{1} w_{2} w_{3}$, $w^{\prime}=w_{2}$ by the pair $w_{1} w_{2}$
$w_{2} w_{3}$ , where the $w_{i}$ satisfy one of the following conditions:

(A) $\left|w_{3}\right|=1 \leqq\left|w_{1}\right|$.

(B) $\quad\left|w_{1}\right|=1 \leqq\left|w_{3}\right|$.

(C) $\left|w_{3}\right|=2$ and $\left|w_{1}\right|=2 i$ with $i \geqq 1$.

Moreover if w" is another word of $D_{Z}$ and if one of the conditions (A), (B), (C) holds for the pair w" instead of the pair w, then we have $\left|w^{\prime \prime}\right| \leqq\left|w^{\prime}\right|$.

We define $K$-invariant polynomials $P_{2 i}(i=1, \ldots, n)$ on $V$ by the following formula:

$$
\operatorname{det}\left(\lambda I_{m+n}+Z\right)=\lambda^{m-n}\left(\lambda^{2 n}+P_{2}(Z) \lambda^{2 n-2}+\cdots+P_{2 n}(Z)\right) \quad(Z \in V) .
$$

It is clear that $J=\boldsymbol{C}\left[P_{2}, \ldots, P_{2 n}\right]$. Using $P_{2}, P_{4}, \ldots, P_{2 n}$, we define the map $\chi: V \rightarrow \boldsymbol{C}^{n}$ as we $\operatorname{did}$ in $\S 1$. It is convenient for our purpose to discuss on the cases $m=n, m>n$, separately.

\section{(3.3. A) The case when $m=n$.}

The matter in hand is the determination of the nilpotent elements 
$Z$ of $V$ with the condition that the differential $(D \chi)_{Z}$ of the map $\chi$ at $Z$ is of rank $\geqq n-1$. The reason why we treat the question is this: If $Z$ is an element of $\mathscr{N}(V)$ and $\operatorname{rank}(D \chi)_{Z} \geqq n-1$, then $\mathscr{S}_{Z} \cap \mathscr{N}(V)$ is non-singular or is a hypersurface of an affine space, where $\mathscr{S}_{Z}$ is a transversal slice of the $K$-orbit of $Z$.

Lemma 3.7. Let $Z$ be an nilpotent element of $\mathscr{N}(V)$ and let $J_{\eta}$ be the Jordan's normal form of $Z$, where $\eta=\left(p_{1}, \ldots, p_{r}\right)\left(p_{1} \geqq \cdots \geqq\right.$ $\left.p_{r} \geqq 0\right)$ is a partition of $m+n$. If $p_{1}<2 n-2$, then $\operatorname{rank}(D \chi)_{Z} \leqq n-2$.

This is a direct consequence of Proposition 3.1.

The following statement is a special case of Proposition 1.18'.

(a) Let $\eta$ be any partition of $2 n$ and let $\mathcal{O}(\eta)$ be the $S L(2 n, \boldsymbol{C})$ orbit of $J_{\eta}$ in $\mathfrak{g}$. Then $\mathcal{O}(\eta) \cap V \neq \phi$.

Let $Z$ be in $\mathscr{N}(V)$ and let $J_{\eta}(\eta \in P(2 n))$ be the Jordan's normal form of $Z$. Assume that $\left(d P_{2}\right)_{Z}, \ldots,\left(d P_{2 n}\right)_{Z}$ are linearly independent. Then $\eta$ is one of the following partitions

$$
(2 n),(2 n-1,1),(2 n-2,2),(2 n-2,1,1) \text {. }
$$

This follows from Lemma 3.3 and the claim (a). The diagrams corresponding to these partitions are as follows:

$$
\begin{aligned}
& |w(12)|,\left|w^{\prime}(21)\right|,\left|\begin{array}{c}
w(1) \\
(2)
\end{array}\right|,\left|\begin{array}{c}
w^{\prime}(2) \\
(1)
\end{array}\right| \\
& \left|\begin{array}{l}
w \\
(12)
\end{array}\right|,\left|\begin{array}{c}
w \\
(21)
\end{array}\right|,\left|\begin{array}{c}
w^{\prime} \\
(21)
\end{array}\right|,\left|\begin{array}{c}
w^{\prime} \\
(12)
\end{array}\right|,\left|\begin{array}{c}
w \\
(1) \\
(2)
\end{array}\right|,\left|\begin{array}{c}
w^{\prime} \\
(1) \\
(2)
\end{array}\right| .
\end{aligned}
$$

Here $w=(\overbrace{1212 \ldots 1}^{2 n-2})$ and $w^{\prime}=(\overbrace{2121 \ldots 2}^{2 n-2} 1)$. It is clear that for any diagram in (3.3. A.1), there exists a nilpotent element of $V$ with the given one. We give here a remark. Let $Z$ and $Z^{\prime}$ be in $\mathcal{N}(V)$ and $D_{Z}$ and $D_{Z^{\prime}}$ be the diagrams of $Z$ and $Z^{\prime}$, respectively. Then $Z$ and $Z^{\prime}$ are $K_{\theta}$-conjugate if and only if $D_{Z^{\prime}}$ coincides with $D_{z}$ up to permutation of words or is obtained from $D_{Z}$ by changing 1 and 2 in all the words of $D_{z}$.

Proposition 3. 8. Let $Z$ be a nilpotent element of $\mathscr{N}(V)$ such that the diagram of $Z$ is one of the diagrams in (3.3. A.1). Let $\mathscr{S}_{Z}$ be the transversal slice defined in $\S 2$. Then $\overline{\mathscr{S}}_{Z}=\mathscr{S}_{Z} \cap \mathcal{N}(V)$ is expressed 
as in Table IV.

Table IV

\begin{tabular}{|c|c|c|}
\hline & $D_{z}$ & $\overline{\mathscr{S}_{Z}}$ \\
\hline ( 1 ) & $|w(12)| \quad\left|w^{\prime}(21)\right|$ & one point \\
\hline (2) & $\left|\begin{array}{c}w(1) \\
(2)\end{array}\right|, \quad\left|\begin{array}{c}w^{\prime}(2) \\
(1)\end{array}\right|$ & $\{(x, y) ; x y=0\}$ \\
\hline ( 3 ) & $\left|\begin{array}{l}w \\
(12)\end{array}\right|, \quad\left|\begin{array}{l}w^{\prime} \\
(21)\end{array}\right|$ & $\left\{(x, y, z) ; x^{n}+y z=0\right\}$ \\
\hline (4) & $\left|\begin{array}{l}w \\
(21)\end{array}\right|,\left|\begin{array}{l}w^{\prime} \\
(12)\end{array}\right|$ & $\left\{(x, y, z) ; x\left(x^{n-1}+y z\right)=0\right\}$ \\
\hline ( 5 ) & $\left|\begin{array}{c}w \\
(1) \\
(2)\end{array}\right|,\left|\begin{array}{c}w^{\prime} \\
(2) \\
(1)\end{array}\right|$ & $\left\{(x, y, z, u) ;(x u)^{n}+x y z=0\right\}$ \\
\hline
\end{tabular}

We can obtain the proposition by a straightforward calculation.

The following proposition gives an answer to Problem (3-III)$(3-\mathrm{V})$.

\section{Proposition 3.9.}

(1) Define the $K_{\theta}$-orbits $\mathcal{O}_{1}, \mathcal{O}_{2}$ of $\mathscr{N}(V)$ by

$$
\begin{aligned}
& \mathcal{O}_{1}=\left\{Z \in \mathscr{N}(V) ; D_{Z} \text { is one of }\left|\begin{array}{c}
w(1) \\
(2)
\end{array}\right|,\left|\begin{array}{c}
w^{\prime}(2) \\
(1)
\end{array}\right|\right\}, \\
& \mathcal{O}_{2}=\left\{Z \in \mathscr{N}(V) ; D_{Z} \text { is one of }\left|\begin{array}{c}
w \\
(12)
\end{array}\right|,\left|\begin{array}{l}
w^{\prime} \\
(21)
\end{array}\right|\right\} .
\end{aligned}
$$

Then $\mathscr{N}(V)_{\text {sing }}^{\prime}=\mathcal{O}_{1} \cup \mathcal{O}_{2}$.

(2) For any $Z \in \mathcal{O}_{1}, \pi^{-1}\left(\overline{\mathscr{S}_{Z}}\right)$ is the disjoint union of two affine lines.

(3) For any $Z \in \mathcal{O}_{2}$, the va riety $\overline{\mathscr{S}_{Z}}$ is biholomorphic to the surface $\left\{(x, y, z) \in \boldsymbol{C}^{3} ; x^{n}+y z=0\right\}$ and $\pi$ induces a resolution $\pi^{-1}\left(\overline{\mathscr{S}_{Z}}\right) \rightarrow \overline{\mathscr{S}_{Z}}$, which is minimal. In particular, the fibre $\pi^{-1}(Z)$ is a Dynkin curve of type $A_{n-1}$.

Proof. The claim (1) follows from Lemma 3.6 and (2) is easily shown. 
We prove (3). Let $Z=\left(\begin{array}{ll}0 & X \\ Y & 0\end{array}\right)$ with $X=I_{n}$ and $Y=\left(\begin{array}{ll}J_{n-1} & 0 \\ 0 & 0\end{array}\right)$. Then $Z$ is obviously contained in $\mathscr{O}_{2}$. We take a transversal slice to $\mathcal{O}_{2}$ at $Z$ different from $\mathscr{S}_{Z}$. Define $\mathscr{S}_{Z}^{\prime}=Z+U_{Z}$, where

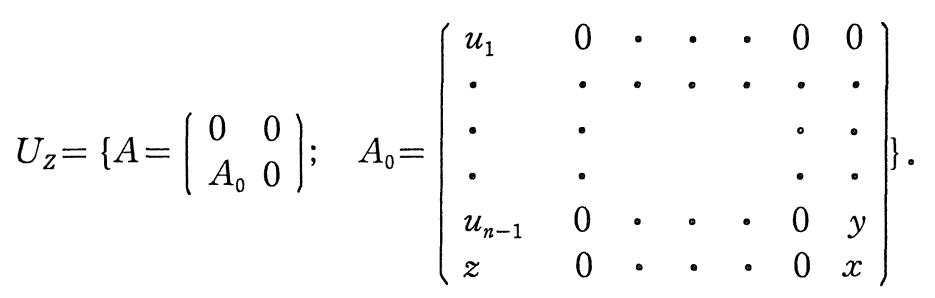

By direct calculation, we find that $V=[k, Z]+U_{Z}$ is a direct sum decomposition. This implies that $\mathscr{S}_{Z}$ is a transversal slice to $\mathcal{O}_{2}$ at $Z$. The choice of a transversal slice is not essential to prove (3). The only thing to be noted is that the restriction of $\chi$ to it preserves the quasihomogeneous structure. By direct calculation, we find that $Z+A$ $\left(A \in U_{Z}\right)$ is nilpotent if and only if $u_{i}=-x^{i}(1 \leqq i \leqq n-1)$ and $x^{n}+$ $y z=0$. Hence $\overline{\mathscr{S}_{Z}^{\prime}}=\mathscr{S}_{Z}^{\prime} \cap \mathscr{N}(V)=\left\{(x, y, z) ; x^{n}+y z=0\right\}$. Let $Z_{1}=$ $\left(\begin{array}{cc}0 & I_{n} \\ J_{n} & 0\end{array}\right)$ and $H_{1}=\operatorname{diag}\left(t_{1}, \ldots, t_{n}, u_{1}, \ldots, u_{n}\right)$ with $t_{i}=2 n-4 i+3, u_{i}=$ $2 n-4 i+1(1 \leqq i \leqq n)$. Then $Z_{1}$ is principal nilpotent of $\mathscr{N}(V)$ and there exists a unique element $Y_{1}$ of $V$ such that $\left(H_{1}, Z_{1}, Y_{1}\right)$ is a normal $S$-triple. As we did in $\S 2$, we constructed the manifold $\tilde{\mathscr{N}}(V)$ by using $\left(H_{1}, Z_{1}, Y_{1}\right)$. Furthermore, we let $G_{0}=S L(n, C)$ and $B=$ \{upper triangular matrices in $G_{0}$ \}. Let $\mathfrak{n}_{0}$ be the nilpotent radical of the Lie algebra of $B$. Then we define the homogeneous vector bundle $G_{0} \times{ }^{B} \mathfrak{n}_{0}$ as in $\S 2$. For brevity, we present the mapping of $G_{0} \times{ }^{B} \mathfrak{n}_{0}$ to $\mathfrak{g}_{1}=\mathfrak{g l}(n, \boldsymbol{C})$ by $\pi_{0}$ which is obviously defined by $\pi_{0}(g * A)$

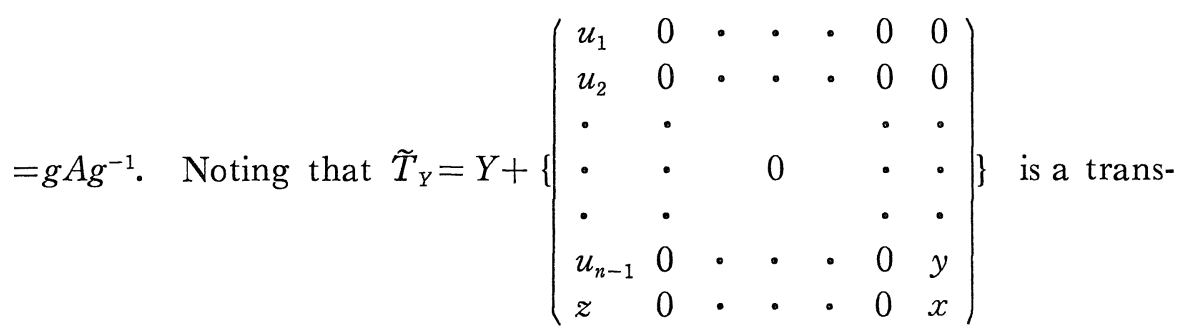

versal slice to the $G_{0}$-orbit of $Y$ in $g_{1}$, we find that $\pi^{-1}\left(\mathscr{S}_{Z}^{\prime}\right)=\pi_{0}^{-1}\left(T_{Y}\right)$, where $T_{Y}=\left\{Y_{1} \in \widetilde{T}_{Y} ; Y_{1}\right.$ is nilpotent $\}$. Since $Y$ is a subregular nilpotent element of $\mathrm{g}_{1}$, it follows from [Sl] that $\pi_{0}^{-1}\left(T_{Y}\right) \rightarrow T_{Y}$ is the minimal resolution. The rest of the claims are nearly obvious. q.e.d. 


\section{(3.3. B) The case when $m \geqq n+1$.}

For convenience, we first determine such nilpotent elements of $V$ that the differential $D \chi$ is of rank $\geqq n-1$ at the points. By an argument similar to the one in (3.3. A), we conclude that if $Z$ is nilpotent of $\mathcal{N}(V)$ and if $J_{\eta}(\eta \in P(m+n))$ is the Jordan's normal form of $Z$, then $\eta$ is one of the following partitions $(p=m-n)$.

(3. 3. B. 1) $\left(2 n+1,1^{p-1}\right),\left(2 n, 1^{p}\right),\left(2 n-1,3,1^{p-2}\right),\left(2 n-1,2,1^{p-1}\right)$, $\left(2 n-1,1^{p+1}\right),\left(2 n-2,3,1^{p-1}\right),\left(2 n-2,2,1^{p}\right),\left(2 n-2,1^{p+2}\right)$.

Noting this, we obtain the following proposition.

Proposition 3.10. Let $Z$ be a nilpotent element of $\mathcal{N}(V)$ such that $J_{\eta}(\eta \in P(m+n))$ is the Jordan's normal form of $Z$, where $\eta$ is one of the partitions in (3.3.B.1). Let $\mathscr{S}_{Z}$ be the transversal slice as defined in $\S 2$. Then the diagram $D_{z}$ of $Z$ is one of those in Table $V$ and $\overline{\mathscr{S}_{Z}}=\mathscr{S}_{Z} \cap \mathscr{N}(V)$ is expressed as there.

The proof of this proposition is straightforward. Hence we omit it.

Define an outer automorphism $a$ of $\mathfrak{g}$ by $a(Z)=-{ }^{t} Z(Z \in \mathfrak{g})$ and denote by $K^{\prime}$ the group generated by $K$ and $a$. Then $K^{\prime}$ plays a role similar to $K_{\theta}$ in (3.3. A). In particular, if $Z$ and $Z^{\prime}$ are nilpotents of $\mathscr{N}(V)$ such that $D_{Z}$ and $D_{Z^{\prime}}$ are the diagrams contained in the same box in Table (3-II). Then $Z$ and $Z^{\prime}$ are $K^{\prime}$-conjugate. By this reason, it is convenient to consider $K^{\prime}$-orbits instead of $K$-orbits.

\section{Proposition 3. 11.}

(1) Define the $K^{\prime}$-orbits $\mathcal{O}_{1}$ and $\mathcal{O}_{2}$ of $\mathcal{N}(V)$ by

$$
\begin{aligned}
& \mathcal{O}_{1}=\left\{Z \in \mathscr{N}(V) ; D_{Z} \text { is }\left|\begin{array}{c}
w(121) \\
(1)^{p-1}
\end{array}\right|\right\} \\
& \mathcal{O}_{2}=\left\{Z \in \mathscr{N}(V) ; D_{Z} \text { is one of }\left|\begin{array}{c}
w(12) \\
(1)^{p}
\end{array}\right|,\left|\begin{array}{c}
w^{\prime}(21) \\
(1)^{p}
\end{array}\right|\right\} .
\end{aligned}
$$

(For the definition of $w$ and $w^{\prime}$, see Table $V$ ). Then $\mathcal{O}_{1}$ coincides with $\mathscr{N}(V)_{p r}$ and $\mathscr{N}(V)_{\text {reg }}=\mathcal{O}_{1} \cup \mathcal{O}_{2}$.

Moreover, if $Z$ is contained in $\mathcal{O}_{2}$, then $\pi^{-1}\left(\overline{\mathscr{S}_{Z}}\right)$ coincides with the blowing up of the affine space $\overline{\mathscr{S}}_{Z}^{-}=\boldsymbol{C}^{p}$ with the center at the origin.

(2) Define the $K^{\prime}$-orbits $\mathcal{O}_{3}, \mathcal{O}_{4}, \mathcal{O}_{5}$ of $\mathscr{N}(V)$ by 


$$
\begin{aligned}
& \mathcal{O}_{3}=\left\{Z \in \mathcal{N}(V) ; D_{Z} \text { is }\left|\begin{array}{c}
w^{\prime}(2) \\
(1)^{p+1}
\end{array}\right|\right\} \\
& \mathcal{O}_{4}=\left\{Z \in \mathcal{N}(V) ; D_{Z} \text { is }\left|\begin{array}{c}
w(1) \\
(121) \\
(1)^{p-2}
\end{array}\right|\right\} \\
& \mathcal{O}_{5}=\left\{Z \in \mathcal{N}(V) ; D_{Z} \text { is one of }\left|\begin{array}{c}
w(1) \\
(12) \\
(1)^{p-1}
\end{array}\right|,\left|\begin{array}{c}
w(1) \\
(21) \\
(1)^{p-1}
\end{array}\right|\right\} .
\end{aligned}
$$

If $m=n+1$, then $\mathscr{N}(V)_{\text {sing }}^{\prime}=\mathcal{O}_{3} \cup \mathcal{O}_{5}$ and $\mathcal{O}_{4}=\phi$. On the other hand, if $m \geqq n+2$, then $\mathcal{N}(V)_{\text {sing }}^{\prime}=\mathcal{O}_{3} \cup \mathcal{O}_{4}$ and $\mathcal{O}_{5} \subsetneq \overline{\mathcal{O}}_{4}$.

(3) For any $Z \in \mathcal{O}_{3}, \pi^{-1}\left(\mathscr{S}_{Z}\right)$ is biholomorphic to the blowing up of the quadratic hypersurface defined in Example 1.14 (continued) in $\S 2$ by substituting $n$ by $p$.

(4) For any $Z \in \mathcal{O}_{5}$ if $m=n+1$ (resp. $Z \in \mathcal{O}_{4}$ if $m \geqq n+2$ ), the variety $\overline{\mathscr{S}}_{Z}$ is nothing but the simple surface singularity of type $A_{n-1}$ and $\pi$ induces a resolution $\pi^{-1}\left(\overline{\mathscr{S}_{Z}}\right) \rightarrow \overline{\mathscr{S}_{Z}}$, which is minimal.

The proof of (1) is easy. The rest of the claims are similar to

\begin{tabular}{|c|c|c|c|}
\hline & \multicolumn{2}{|c|}{$D_{z}$} & $\overline{\mathscr{S}_{Z}}$ \\
\hline ( 1 ) & $\left|\begin{array}{l}w(121) \\
(1)^{p-1}\end{array}\right|$ & & one point \\
\hline ( 2 ) & $\left|\begin{array}{c}w(12) \\
(1)^{p}\end{array}\right|$ & $\begin{array}{l}w^{\prime}(21) \\
(1)^{p}\end{array} \mid$ & $\boldsymbol{C}^{p}$ \\
\hline ( 3 ) & $\left|\begin{array}{c}w(1) \\
(121) \\
(1)^{p-2}\end{array}\right|$ & & $\left\{(x, y, z) \in \boldsymbol{C}^{3} ; x^{n}+y z=0\right\}$ \\
\hline$(3)^{\prime}$ & $\left|\begin{array}{l}w(1) \\
(12) \\
(1)^{p-1}\end{array}\right|$, & $\begin{array}{l}w(1) \\
(21) \\
(1)^{p-1}\end{array}$ & $\left\{(x, y, z) ; x^{n}+y z=0\right\} \times \boldsymbol{C}^{p-1}$ \\
\hline (4) & $\mid \begin{array}{l}w^{\prime}(2) \\
(1)^{p+1}\end{array}$ & & $\begin{array}{l}\left\{(u, v) \in \boldsymbol{C}^{p+1} \times \boldsymbol{C}^{p+1} ;\right. \\
\left.u_{1} v_{1}+\cdots+u_{p+1} v_{p+1}=0\right\}\end{array}$ \\
\hline
\end{tabular}
those of Propositions 3.3. Hence the proof is omitted.

Table V 


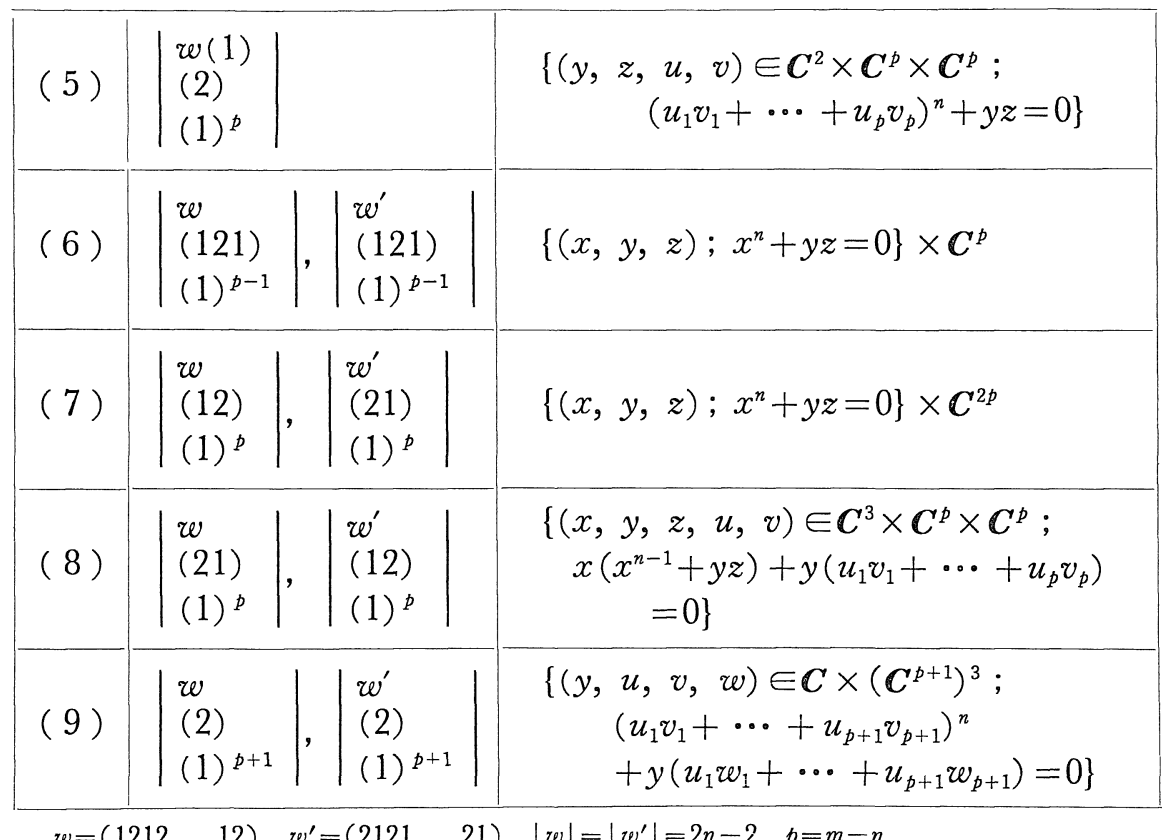

$w=(1212 \ldots 12), \quad w^{\prime}=(2121 \ldots 21), \quad|w|=\left|w^{\prime}\right|=2 n-2, \quad p=m-n$

\section{§4. The Generic Singularity of the Nilpotent Subvariety The Classical Case}

In this section we treat the nilpotent variety $\mathcal{N}(V)$ when the corresponding symmetric pair $(\mathfrak{g}, \mathfrak{f})$ is of classical type. We mainly discuss on Problems (3-III) and (3-IV). The results of this section are quite incomplete but the author hopes that they will be useful for the further study in this area.

To describe the results, we need some preparation.

(4.1) In this section the Lie algebras $\mathfrak{Z} \mathfrak{l}(n, \boldsymbol{C}), \mathfrak{s p}(n, \boldsymbol{C})$ and $\mathfrak{s} \mathfrak{D}(n, \boldsymbol{C})$ denote the standard matrix algebras (cf. [Hel]).

(4.2) If $X$ is an $n \times n$ nilpotent matrix, we define a partition $\eta(X)$ associated with $X$ as follows. Let $J_{\eta}$ be the Jordan's normal form of $X$. Then $\eta(X)=\eta$. (Here we used the notation in $\S 3$ ).

(4.3) Let $\eta=\left(p_{1}, \ldots, p_{k}\right)$ be a partition of $n$. Then we define $\eta^{2}=\left(p_{1}, p_{1}, p_{2}, p_{2}, \ldots, p_{k}, p_{k}\right)$. This is a partition of $2 n$. (Cf. § 3.)

(4.4) Let $X$ be a nilpotent element of $V$. Then $\mathscr{S}_{X}$ denotes the transversal slice of the $K$-orbit of $X$ defined in Lemma 1.21. Moreover we put $\overline{\mathscr{S}_{X}}=\mathscr{S}_{X} \cap \mathcal{N}(V)$. 
(4.5) We define an equivalence relation on $\mathscr{N}(V)$. Let $X$ and $X^{\prime}$ be two elements of $\mathscr{N}(V)$. Then $X$ and $X^{\prime}$ are equivalent if and only if $\overline{\mathscr{S}_{X}}$ and $\overline{\mathscr{S}_{X^{\prime}}}$ are isomorphic to each other.

We note here that two equivalent elements of $\mathscr{N}(V)$ are not always $K_{\theta}$-conjugate.

Let $(\mathfrak{g}, \mathfrak{f})$ be a symmetric pair. In this section we attempt to determine all the nilpotent $K$-orbits of $\mathscr{N}(V)$ which are open in $\mathscr{N}(V)_{\text {sing. }}$ Unfortunately, this is not done. But we shall give a weak version of this claim. For this purpose, we define a Zariski open subset $\mathscr{N}(V)_{\text {sing }}^{\prime \prime}$ of $\mathscr{N}(V)_{\text {sing. }}$.

(4.6) Let $\mathscr{N}(V)_{\text {sing }}^{\prime}$ be the non-singular part of $\mathscr{N}(V)_{\text {sing }}$ (cf. Problem (3-III)). We define a subset $\mathscr{N}(V)_{\text {sing }}^{\prime \prime}$ of $\mathscr{N}(V)_{\text {sing. }}^{\prime}$ An element $X$ of $V$ is contained in $\mathscr{N}(V)_{\text {sing }}^{\prime \prime}$ if and only if the following conditions (4.6.1) and (4.6.2) hold.

(4.6.1) $K \cdot X$ is open in $\mathscr{N}(V)_{\text {sing. }}^{\prime}$.

(4.6.2) $\operatorname{rank}(D \chi)_{X} \geqq l-1$. (Here $l$ is the rank of $(\mathfrak{g}, \mathfrak{f})$ and $D \chi$ is the differential of the map $\chi: V \rightarrow \mathfrak{a} / W_{\text {.) }}$

It is not clear whether the condition (4.6.2) holds for any element of $\mathscr{N}(V)_{\text {sing }}^{\prime}$ or not. However the reason why we introduce the set $\mathscr{N}(V)_{\text {sing }}^{\prime \prime}$ is based on the following conjecture.

Conjecture (4.7) Let $X$ be a nilpotent element of $V$. Assume that $K \cdot X$ is open in $\mathscr{N}(V)_{\text {sing. }}$ Then $X$ satisfies the condition (4.6.2).

It seems to be sure that the conjecture holds in general but to prove it we must examine closely the closure relation between two different $K_{\theta}$-orbits of $\mathcal{N}(V)$ (cf. Problem (3-II)). In the previous section, we showed that Conjecture (4.7) actually holds when $\mathfrak{g}$ is of type $A$.

In the rest of this section we mainly treat such a nilpotent element $X$ contained in $\mathscr{N}(V)_{\text {sing. }}^{\prime \prime}$. First we treat the following problem.

(4.8) Enumerate all the nilpotent elements of $V$ with the condition (4.6.2).

This is done in the following way. Let $X$ be in $\mathcal{N}(V)$ and assume that rank $(D \chi)_{X} \geqq l-1$. Due to Proposition 3. 1, we can easily rephrase the condition (4.6.2) to that on the partition $\eta(X)$. Here we used Gerstenhaber's result [G] on the Jordan's normal forms of nilpotent 
elements in $\mathfrak{S} \mathfrak{O}(n, \boldsymbol{C})$ and $\mathfrak{s} \mathfrak{p}(n, \boldsymbol{C})$. Also we note that the Jordan's normal form does not determine uniquely a $K_{\theta}$-orbit of $\mathcal{N}(V)$ in general. On the way of the determination of possible partitions $\eta(X)$ so that $X$ satisfy (4.6.2), we observe that the converse statement of Proposition 3.1 holds in almost all cases but does not hold in general. Namely if $\mathfrak{g}$ is of classical type, we have only one counterexample. We give it here.

Example 4.9. Let us consider the symmetric pair $(\mathfrak{g}, \mathfrak{f})=(\mathfrak{S O}(2 n$, $\boldsymbol{C}), \mathfrak{s 0}(n, \boldsymbol{C})+\mathfrak{s o}(n, \boldsymbol{C})) \quad(n \geqq 4)$. We take a nilpotent element $X$ of $V$ such that $\eta(X)=\left(2 n-3,1^{3}\right)$. It follows from Proposition 1.18 and [G] that such a nilpotent $X$ actually exists in $V$. We use the notation Proposition 3.1. Then by direct calculation, we find that $\frac{1}{2} \tilde{\alpha}\left(H^{\prime}\right)+1=d_{l-1}$ but that $\operatorname{rank}(D \chi)_{X}=l-2$ (cf. (4-III) (3) in Table $\mathrm{VI})$.

Next we consider the following problem.

(4.10) Let $\mathcal{O}_{1}, \ldots, \mathcal{O}_{s}$ be all the $K_{\theta}$-orbits of $\mathscr{N}(V)$ such that for each $i$ and $X \in \mathcal{O}_{i}$, rank $(D \chi)_{X} \geqq l-1$. Then we decide whether the closure of an orbit $\mathcal{O}_{i}$ is an irreducible component of $\mathscr{N}(V)_{\text {sing }}$ or not. We note that in this case $\overline{\mathscr{S}}_{X}$ is a hypersurface of an affine space. The explicit form of $\overline{\mathscr{S}_{X}}$ is useful in the examination of the problem (4.8).

From now on we give all the nilpotent orbits of $V$ each element of which satisfies the condition (4.6.2). Let $(\mathfrak{g}, \mathfrak{f})$ be a symmetric pair such that $\mathfrak{g}$ is simple of classical type. Then by classification, $(\mathfrak{g}, \mathfrak{f})$ is one of the pairs $(4-\mathrm{I})-(4-\mathrm{XI})$ in Table VI except the ones treated in the previous section. Let $X$ be a nilpotent element of $V$ with the condition (4.6.2). In Table VI, we give the defining equation of the intersection $\overline{\mathscr{S}_{X}}$ for such an element $X \in \mathcal{N}(V)$ that (4.6.2) holds for $X$.

We give here some comments on Table VI. Assume that $g$ is realized as a matrix algebra of size $n$. Let $\eta$ be a partition of $n$ such that there exists an element $X$ of $\mathscr{N}(V)$ with $\eta=\eta(X)$. Then the defining equation of $\overline{\mathscr{S}}_{X}$ is written in the right-hand side of $\eta$. Needless to say, we do not distinguish equivalent elements of $\mathscr{N}(V)$ 
in Table VI. In some cases we observe that for given $X, X^{\prime} \in \mathcal{N}(V)$, $\overline{\mathscr{S}}_{X}$ and $\overline{\mathscr{S}}_{X^{\prime}}$ are not isomorphic in spite of that $\eta=\eta(X)=\eta\left(X^{\prime}\right)$. Then to distinguish between $\overline{\mathscr{S}_{X}}$ and $\overline{\mathscr{S}_{X}}$, we use the notation $\eta_{\mathrm{I}}$ and $\eta_{\mathrm{I}}$. The former corresponds to $\overline{\mathscr{S}_{X}}$ and the latter to $\overline{\mathscr{S}_{X}}$. The word "generic" means that the corresponding nilpotent $K_{\theta}$-orbit is open in $\mathscr{N}(V)_{\text {sing }}$.

Comparing Table VI and the results in $\S 3$, we observe the following facts (4.10.1), (4.10.2).

(4.10.1) Let $(\mathfrak{g}, \mathfrak{f})$ be a symmetric pair such that $\mathfrak{g}$ is simple of classical type. Then there are at most two equivalent classes in $\mathscr{N}(V)_{\text {sing }}^{\prime \prime}$.

This observation is already suggested in [S-S]. The author hopes that there is a deep connection between this observation and the root systems of the symmetric pairs.

(4. 10.2) For any $X \in \mathscr{N}(V)_{\text {sing }}^{\prime \prime}$, the intersection $\overline{\mathscr{S}_{X}}$ is a simple singularity in the sense of Arnol'd [A].

Table VI

$$
\begin{aligned}
& \text { (4-I) } \quad(\mathfrak{s} \mathfrak{0}(n+1, \boldsymbol{C}), \mathfrak{g} \mathfrak{D}(n, \boldsymbol{C})) \quad(n \geqq 6) \\
& \text { (1) }\left(3,1^{n-2}\right) \quad \text { one point } \\
& \text { (2) }\left(1^{n+1}\right) \quad x_{1}^{2}+\cdots+x_{n}^{2}=0 \text { (generic) } \\
& (4-\mathrm{II}) \quad(\mathfrak{s} \mathfrak{D}(n+3, \boldsymbol{C}), \mathfrak{b} \mathfrak{D}(n+1, \boldsymbol{C})+\mathfrak{g} \mathfrak{D}(2, \boldsymbol{C})) \quad(n \geqq 3) \\
& \text { (1) }\left(5,1^{n-2}\right) \quad \text { one point } \\
& \text { (2) }\left(3,3,1^{n-3}\right) \quad x y=0 \text { (generic) } \\
& \text { (3) }\left(3,1^{n}\right)_{\mathrm{I}} \quad x_{1}^{2}+\cdots+x_{n}^{2}=0 \text { (generic) } \\
& \text { (4) }\left(3,1^{n}\right)_{\text {II }} \quad(x y)^{2}+z^{2}=0 \\
& \text { (5) }\left(2,2,1^{n-1}\right) \quad(x y)^{2}+y\left(u_{1}^{2}+\cdots+u_{n-1}^{2}\right)=0
\end{aligned}
$$

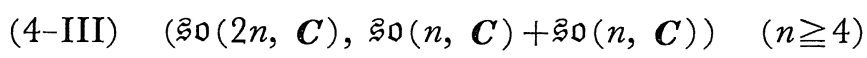

$$
\begin{aligned}
& \text { (1) }(2 n-1,1) \text { one point } \\
& \text { (2) }(2 n-3,3) \quad x^{n-1}+x y^{2}=0 \text { (generic) } \\
& \text { (3) }\left(2 n-3,1^{3}\right) \text { (not hypersurface singularity) } \\
& \left\{\begin{array}{l}
\left(x_{1}^{2}+x_{2}^{2}\right)^{n-1}+y_{1}^{2}+y_{2}^{2}=0 \\
x_{1} y_{1}+x_{2} y_{2}=0
\end{array}\right. \\
& \text { (4-IV) } \quad(\mathfrak{g} \mathfrak{0}(2 n+1, \boldsymbol{C}), \mathfrak{S} \mathfrak{o}(n+1, \boldsymbol{C})+\mathfrak{g} \mathfrak{0}(n, \boldsymbol{C})) \quad(n \geqq 3) \\
& \text { (1) }(2 n+1) \quad \text { one point } \\
& \text { (2) }\left(2 n-1,1^{2}\right)_{\text {I }} \quad x^{2 n}+y^{2}=0 \text { (generic) }
\end{aligned}
$$


( 3 ) $\left(2 n-1,1^{2}\right)_{\text {II }} \quad x y=0$ (generic)

$(4-\mathrm{V}) \quad(\mathfrak{g} \mathfrak{D}(2 n+k, \boldsymbol{C}), \mathfrak{g} \mathfrak{D}(n+k, \boldsymbol{C})+\mathfrak{S} \mathfrak{D}(n, \boldsymbol{C})) \quad(n \geqq 3, k \geqq 2)$

(1) $\left(2 n+1,1^{k-1}\right) \quad$ one point

(2) $\quad\left(2 n-1,3,1^{k-2}\right) \quad x^{n}+y^{2}=0$ (generic)

(3) $\left(2 n-1,1^{k+1}\right)_{\text {I }} \quad x_{1}^{2}+\cdots+x_{k+1}^{2}=0$ (generic)

(4) $\quad\left(2 n-1,1^{k+1}\right)$ II $\quad(u v)^{n}+y^{2}=0$

$(4-\mathrm{VI}) \quad(\mathfrak{g o}(4 n, \boldsymbol{C}), \mathfrak{g}[(2 n, \boldsymbol{C})+\boldsymbol{C}) \quad(n \geqq 2)$

(1) $(2 n)^{2}$

one point

(2) $(2 n-1,1)^{2}$

$x y=0$ (generic)

( 3 ) $(2 n-2,2) \underset{\mathrm{I}}{2}$

$x^{n}+u_{1} v_{1}+u_{2} v_{2}=0$ (generic)

(4) $(2 n-2,2) \frac{2}{\text { II }}$

$x\left(x^{n-1}+u_{1} v_{1}+u_{2} v_{2}\right)=0$

( 5 ) $\left(2 n-2,1^{2}\right)^{2}$

$(x y)^{n}+x\left(u_{1} v_{1}+u_{2} v_{2}\right)=0$

$(4-\mathrm{VII}) \quad(\mathfrak{g} \mathfrak{D}(4 n+2, \boldsymbol{C}), \mathfrak{g l}(2 n+1, \boldsymbol{C})+\boldsymbol{C}) \quad(n \geqq 2)$

(1) $(2 n+1)^{2}$

one point

(2) $(2 n, 1)^{2}$

$C^{2}$ (smooth)

(3) $(2 n-1,2)^{2}$

$x^{n}+u_{1} v_{1}+u_{2} v_{2}=0$ (generic)

(4) $\quad\left(2 n-1,1^{2}\right)_{\mathrm{I}}^{2}$

$u_{1} v_{1}+u_{2} v_{2}+u_{3} v_{3}=0$ (generic)

( 5 ) $\quad\left(2 n-1,1^{2}\right) \frac{2}{\text { II }}$

$(x y)^{n}+u_{1} v_{1}+u_{2} v_{2}=0$

(6) $(2 n-2,3)^{2}$

$\left\{x^{n}+u_{1} v_{1}+u_{2} v_{2}=0\right\} \times \boldsymbol{C}^{2}$

( 7 ) $(2 n-2,2,1)_{1}^{2}$

$\left\{x^{n}+u_{1} v_{1}+u_{2} v_{2}=0\right\} \times C^{4}$

( 8 ) $(2 n-2,2,1) \underset{\text { II }}{2}$

$u_{1}^{n}+u_{1}\left(v_{2} w_{3}-v_{3} w_{2}\right)$

$$
+u_{2}\left(v_{3} w_{1}-v_{1} w_{3}\right)+u_{3}\left(v_{1} w_{2}-v_{2} w_{1}\right)=0
$$

( 9 ) $\left(2 n-2,1^{3}\right)^{2}$

$$
\left(t_{1} u_{1}+t_{2} u_{2}+t_{3} u_{3}\right)^{n}+u_{1}\left(v_{2} w_{3}-v_{3} w_{2}\right)
$$$$
+u_{2}\left(v_{3} w_{1}-v_{1} w_{3}\right)+u_{3}\left(v_{1} w_{2}-v_{2} w_{1}\right)=0
$$

$(4-\mathrm{VIII}) \quad(\mathfrak{g p}(n, \boldsymbol{C}), \mathfrak{g l}(n, \boldsymbol{C})+\boldsymbol{C}) \quad(n \geqq 2)$

(1) $(2 n)$

$$
\text { one point }
$$

( 2) $(2 n-2,2)_{\mathrm{I}}$

$x^{2 n}+x y^{2}=0$ (generic)

(3) $(2 n-2,2)_{\text {II }}$

$x^{2 n}+y^{2}=0$ (generic)

(4) $\left(2 n-2,1^{2}\right)$

$(x w)^{2 n}+x y^{2}=0$

$(4-\mathrm{IX}) \quad(\mathfrak{s p}(2 n, \boldsymbol{C}), \mathfrak{s p}(n$,

(1) $(2 n)^{2}$

(2) $(2 n-1,1)^{2}$

(3) $(2 n-2,2)^{2}$

(4) $\left(2 n-2,1^{2}\right)^{2}$
$\boldsymbol{C})+\mathfrak{s p}(n, \boldsymbol{C})) \quad(n \geqq 2)$

one point

$x y+z w=0$ (generic)

$x\left(x^{n-1}+u v\right)+y z=0$

$\left(x_{1} x_{3}-x_{2} x_{4}\right)^{n}+x_{1} u_{2} u_{3}+x_{2} u_{3} u_{4}$

$$
+x_{3} u_{4} u_{1}+x_{4} u_{1} u_{2}=0
$$




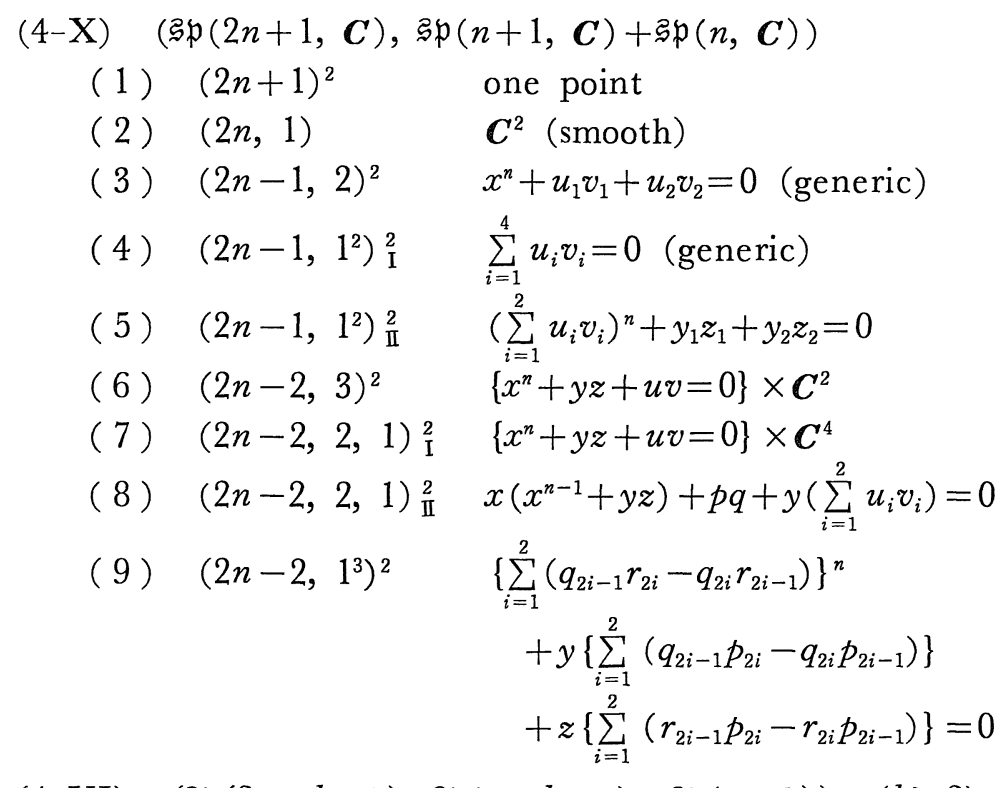

$(4-\mathrm{XI}) \quad(\mathfrak{s p}(2 n+k, \boldsymbol{C}), \mathfrak{s p}(n+k, \boldsymbol{C})+\mathfrak{g p}(n, \boldsymbol{C})) \quad(k \geqq 2)$
(1) $\left(2 n+1,1^{k-1}\right)^{2}$
one point
(2) $\left(2 n, 1^{k}\right)^{2}$
$\boldsymbol{C}^{2 k}$ (smooth)
( 3 ) $\left(2 n-1,3,1^{k-2}\right)^{2}$
$x^{n}+y z+u v=0$ (generic)
(4) $\left(2 n-1,2,1^{k-1}\right)^{2}$
$\left\{x^{n}+y z+u v=0\right\} \times C^{2 k-2}$
(5) $\left(2 n-1,1^{k+1}\right)_{\mathrm{I}}^{2}$
$\sum_{i=1}^{2 k+2} u_{i} v_{i}=0$ (generic)
(6) $\left(2 n-1,1^{k+1}\right) \frac{2}{\text { II }}$
$\left(\sum_{i=1}^{2 k} u_{i} v_{i}\right)^{n}+y z+u v=0$
( 7 ) $\left(2 n-2,3,1^{k-1}\right)^{2}$
$\left\{x^{n}+y z+u v=0\right\} \times C^{2 k}$
( 8 ) $\left(2 n-2,2,1^{k}\right)_{\mathrm{I}}^{2}$
$\left\{x^{n}+y z+u v=0\right\} \times C^{4 k}$
( 9 ) $\left(2 n-2,2,1^{k}\right)_{\text {II }}^{2}$
$x\left(x^{n-1}+y z\right)+p q+y\left(\sum_{i=1}^{\stackrel{N}{N}} u_{i} v_{i}\right)=0$
(10) $\left(2 n-2,1^{k+2}\right)^{2}$

$$
\begin{aligned}
& \left\{\sum_{i=1}^{k+1}\left(q_{2 i-1} r_{2 i}-q_{2 i} r_{2 i-1}\right)\right\}^{n} \\
& +y\left\{\sum_{i=1}^{k+1}\left(q_{2 i-1} p_{2 i}-q_{2 i} p_{2 i-1}\right)\right\} \\
& +z\left\{\sum_{i=1}^{k+1}\left(r_{2 i-1} p_{2 i}-r_{2 i} p_{2 i-1}\right)\right\}=0 .
\end{aligned}
$$

\section{References}

[A] Arnol'd, V. I., Normal forms for functions near degenerate critical points, the Weyl groups $A_{k}, D_{k}, E_{k}$ and Lagrangian singularities, Func. Anal. Appl., 6 (1972), 254-272. 
[B-K] Bala, P. and Carter, R. W., Classes of unipotent elements in simple algebraic groups, I, Proc. Cambridge Philos. Soc., 79 (1976), 401-425.

[B] Berger, M., Les espaces symétriques non compacts, Ann. Sci. Ecole Norm. Sup., 74 (1957), 85-177.

[Br] Brieskorn, E., Singular elements of semisimple algebraic groups, in Actes Congrès Intern. Math., t. 2 (1970), 279-284.

[D] Dynkin, E. B., Semisimple subalgebras of semisimple Lie algebras, Amer. Math. Soc. Transl., (2) 6 (1957), 111-244.

[E] Elkington, G. B., Centralizers of unipotent elements in semisimple algebraic groups, J. of Algebra, 23 (1972), 137-163.

[G] Gerstenhaber, M., Dominance over the classical groups, Ann. of Math., 74 (1961), 532-569.

[H-C] Harish-Chandra, Invariant distributions on Lie algebras, Amer. J. Math., 86 (1964), 271-309.

[Hel] Helgason, S., Differential Geometry and Symmetric spaces, Academic Press (1962) 50.

[Hes 1] Hesselink, W., Singularities in the nilpotent scheme of a classical group, Trans. Amer. Math. Soc., 222 (1976), 1-32.

[Hes 2] - Polarizations in the classical groups, Math. Z., 160 (1978), 217-234.

[K] Kempf, G., On the collapsing of homogeneous bundles, Invent. Math., 37 (1976), 229-239.

[Ke] Kempken, G., Representations of vector space crowns, preprint, Bonn (1979).

[Ko 1] Kostant, B., The three-dimensional subgroup and the Betti numbers of a complex simple Lie group, Amer. J. Math., 81 (1959), 973-1032.

[Ko 2] Lie group representations on polynomial rings, Amer. J. Math., 85 (1963), $327-404$.

[K-R] Kostant, B. and Rallis, S., Orbits and representations associated with symmetric spaces, Amer. J. Math., 93 (1971), 753-809.

[K-P 1] Kraft, H. and Procesi, C., Minimal singularities in $G L_{n}$, Invent. Math., 62 (1981), 503-515.

[K-P 2] - On the geometry of conjugacy classes in classical groups, preprint, Bonn/Rom (1980).

[LIE] Bourbaki, N., Groupes et Algèbres de Lie, IV-VI, Herman, Paris, 1968.

[M 1] Mizuno, K., The conjugate classes of Chevalley groups of type $E_{6}, J$. Fac. Sci. Univ. Tokyo, 24 (1977), 525-563.

[M 2] The conjugate classes of unipotent elements of the Chevalley groups $E_{7}$ and $E_{8}$, Tokyo J. Math., 3 (1980), 391-459.

[O-S] Oshima, T. and Sekiguchi, J., Eigenspaces of invariant differential operators on an affine symmetric space, Invent. Math., 57 (1980), 1-81.

[S-K] Sato, M. and Kimura, T., A classification of irreducible prehomogeneous vector spaces and their relative invariants, Nagoya Math. J., 65 (1977), 1-155.

[S-S] Sekiguchi, J. and Shimizu, Y., Simple singularities and infinitesimally symmetric spaces, Proc. of Japan Acad., 57 Ser. A (1981), 42-46.

[Sl] Slodowy, P., Simple Singularities and Simple Algebraic Groups, Lecture Notes in Math., 815, Springer, Berlin-Heidelberg-New York, 1980.

[Sp] Springer, T. A., The unipotent variety of a semisimple group, Proc. of the Bombay Colloqu. in Algebraic Geometry, ed. S. Abhyanker, London, Oxford Univ. Press, 1969, 373-391.

[Sp-St] Springer T. A. and Steinberg R., Conjugacy classes, in Borel et al.: Seminar on Algebraic Groups and Related Finite Groups, Lecture Notes in Math., 131, Springer, Berlin-Heidelberg-New York, 1970. 
[St] Steinberg, R., Conjugacy Classes in Algebraic Groups, Lecture Notes in Math., 366, Springer, Berlin-Heidelberg-New York, 1974.

[V] Vinberg, E. B., On the classification of the nilpotent elements of graded Lie algebras, Soviet Math. Dokl., 16(6) (1975), 1517-1520.

[W] Warner, G., Harmonic Analysis on Semi-Simple Lie Groups, I, Springer-Verlag, Berlin-Heidelberg-New York, 1972. 